\title{
ANÁLISE DE OUTLIERS, QUEBRAS ESTRUTURAIS \\ E INFLUÊNCIA EM PROCESSOS DE VOLATILIDADE ESTOCÁSTICA
}

\author{
Anderson Carlos Oliveira Motta
}

TESE APRESENTADA

$\mathrm{AO}$

INSTITUTO DE MATEMÁTICA E ESTATÍSTICA

DA

UNIVERSIDADE DE SÃO PAULO

PARA OBTENÇÃO DO GRAU

DE

DOUTOR EM CIÊNCIAS

Área de Concentração: Estatística

Orientador: Prof. Dr. Luiz Koodi Hotta

São Paulo, Outubro de 2006 


\section{ANÁLISE DE OUTLIERS, QUEBRAS ESTRUTURAIS E INFLUÊNCIA EM PROCESSOS DE VOLATILIDADE ESTOCÁSTICA}

Este exemplar corresponde à redação final da tese devidamente corrigida e defendida por Anderson Carlos Oliveira Mota e aprovada pela comissão julgadora.

São Paulo, 05 de outubro de 2006.

Banca Examinadora:

- Prof. Dr. Luiz Koodi Hotta (Orientador) - IMECC/UNICAMP

- Prof. Dr. Pedro Alberto Morettin - IME/USP

- Prof. Dr. Pedro Luiz Valls Pereira - IBMEC/SP

- Prof. Dra. Silvia Regina Costa Lopes - DEST/UFRGS

- Prof. Dr. Cristiano Augusto Coelho Fernandes - PUC/RJ 


\section{Agradecimentos}

À FAPESP (Fundação de Amparo à Pesquisa do Estado de São Paulo) pelo financiamento deste projeto durante o período de 48 meses.

Agradeço ao meu orientador, Prof. Dr. Luiz Koodi Hotta, pela (muita) paciência, amizade e exemplo de dedicação.

Agradeço ao meu irmão Cláudio Fabiano Motta Toledo; o responsável por essa conquista. À minha irmã Valdinéia e Adriana, pelo carinho.

À minha tia Shirley pela dedicação e carinho.

À Sueli Marangoni Domingues pelo carinho e incentivo em todos os momentos.

São Paulo

Anderson Motta

5 de Outubro de 2006 
Dedico esse trabalho à minha Avó Esther Motta (in Memorian) 


\section{SUMÁRIO}

1 Introdução 1

2 O Modelo de VE com Outliers 5

2.1 Modelo de Volatilidade Estocástica . . . . . . . . . . . . . . 6

2.1 .1 Modelo Clássico . . . . . . . . . . . . . . . . . . . . 8

2.1 .2 Modelo Bayesiano . . . . . . . . . . . . . . . . . . 10

2.2 Séries Simuladas . . . . . . . . . . . . . . . . . . . . 12

2.2.1 Série Sem Outliers e Mudanças Estruturais . . . . . . . . . . . 12

2.2.2 Série Com Outlier e Mudanças Estruturais . . . . . . . . . 16

2.3 Séries Empíricas . . . . . . . . . . . . . . . . . . . . . . . . . . . 19

2.3 .1 Série do IBOVESPA $\ldots \ldots \ldots \ldots \ldots$

2.3 .2 Série da NYSE . . . . . . . . . . . . . . . . . . . 22

2.4 Conclusões . . . . . . . . . . . . . . . . . . . . . . . . . 23

3 Detecção de Blocos de Outliers em Modelos de Volatilidade Es$\begin{array}{ll}\text { tocástica } & 25\end{array}$

3.1 Outliers em Modelos de Volatilidade . . . . . . . . . . . . . 26 
3.2 Deteç̧ão e Estimação de Blocos de Outliers . . . . . . . . . . . . . 29

3.2.1 Modelo de Volatilidade Estocástica Bayesiano com Outliers . . 29

3.2.2 Procedimento para Detecção de Blocos . . . . . . . . . . . . . 32

3.2.3 Distribuições a Posteriori para Blocos de Outliers . . . . . . . 33

3.3 Estimação dos Parâmetros do Modelo . . . . . . . . . . . . . . . . 36

3.3.1 Prioris para os Parâmetros do Modelo . . . . . . . . . . 36

3.3.2 Distribuições Posterioris dos Parâmetros . . . . . . . . . . 37

3.4 Aplicações . . . . . . . . . . . . . . . . . . . . . . . . . . 39

3.4 .1 Séries Simuladas . . . . . . . . . . . . . . . . 39

3.4 .2 Análise da Série do Ibovespa . . . . . . . . . . . . . . . 49

3.4.3 Análise da Série da NYSE . . . . . . . . . . . . . . . . . 54

3.5 Conclusões . . . . . . . . . . . . . . . . . . . . . 58

4 Estatística de Influência em Modelos de Volatilidade $\quad 59$

4.1 Análise de Influência . . . . . . . . . . . . . . . . . . . 60

4.2 Estatística para Diagnosticar Influência . . . . . . . . . . . 62

4.2.1 Estatística para Modelo de Volatilidade Estocástica . . . . . . 65

4.3 Propriedades da Estatística para o Modelo de Volatilidade . . . . . . 68

4.3.1 Distribuição de Referência e Poder . . . . . . . . . . . . 68

4.4 Aplicações . . . . . . . . . . . . . . . . . . . 75

4.4 .1 Séries Simuladas . . . . . . . . . . . . . . 75

4.4 .2 Análise da Série do Ibovespa . . . . . . . . . . . . . . 79

4.4 .3 Análise da Série da NYSE . . . . . . . . . . . . . . . . . 84

4.5 Conclusões . . . . . . . . . . . . . . . . . . . . . . 89 
5 Influência Local em Modelos de Volatilidade Estocástica 91

5.1 Influência Local . . . . . . . . . . . . . . . . . . . . . . 92

5.1 .1 Superfície de Influência . . . . . . . . . . . . . . . . . . 94

5.1 .2 Análise da Superfície de Influência . . . . . . . . . . 97

5.2 Procedimento para Determinação de Valores Influentes . . . . . . . 105

5.3 Influência Local em Modelos de VE . . . . . . . . . . . . . . . . . 107

5.3.1 Cálculo da Verossimilhança . . . . . . . . . . . . . 108

5.3 .2 Perturbação das Observações . . . . . . . . . . . . . . . 112

5.3 .3 Perturbação do Modelo . . . . . . . . . . . . . . . . . . . . . 115

5.4 Aplicação da Análise de Influência Local . . . . . . . . . . . . . 118

5.4.1 Estimativas dos Valores Limiares . . . . . . . . . . . . . . 118

5.4 .2 Análise da Série Ibovespa $\ldots \ldots$. . . . . . . . . . . 123

5.4 .3 Análise da Série NYSE . . . . . . . . . . . . . . . . . . 127

5.5 Conclusões . . . . . . . . . . . . . . . . . . . . . 131

6 Conclusão 133

$\begin{array}{ll}\text { Referências } & 141\end{array}$ 



\section{LISTA DE TABELAS}

2.1 Conjunto de parâmetros para o processo gerador de dados. . . . . . . 13

2.2 Estatísticas descritivas das séries simuladas. . . . . . . . . . . . . 14

2.3 Estimativas dos parâmetros para série simulada com conjunto de parâmetros

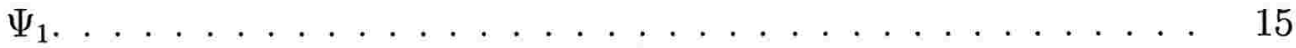

2.4 Estimativas dos parâmetros para série simulada com conjunto de parâmetros

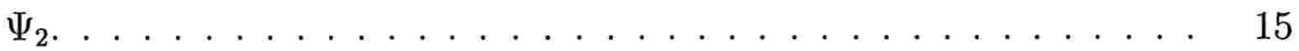

2.5 Conjunto de parâmetros para o processo gerador de dados: $\Delta_{(t, \kappa)}$ é o tamanho dos outliers do tempo $t$ até o tempo $t+\kappa \ldots \ldots \ldots$

2.6 Estatísticas descritivas das séries simuladas com outliers. . . . . . . . 17

2.7 Estimativas dos parâmetros para série simulada com conjunto de parâmetros

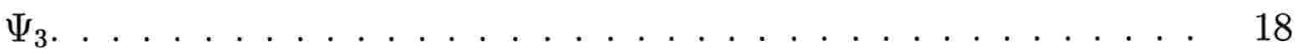

2.8 Estimativas dos parâmetros para série simulada com conjunto de parâmetros

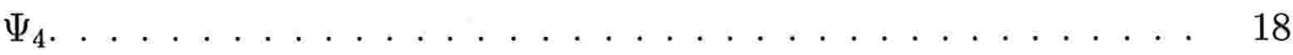

2.9 Estatísticas descritivas dos retornos do Ibovespa. . . . . . . . . . . . . 20

2.10 Estimativas dos parâmetros para Ibovespa. . . . . . . . . . . . . . . . 21 
2.11 Estatísticas descritivas dos retornos da NYSE. . . . . . . . . . . . . 22

2.12 Estimativas dos parâmetros para NYSE. . . . . . . . . . . . . . 23

3.1 Média das distribuições a posteriori dos parâmetros para $\Psi_{1}$ e $\Psi_{3}$ e desvios padrões entre parênteses. . . . . . . . . . . . . . 46

3.2 Médias das distribuições a posteriori dos tamanhos dos outliers e desvios, tamanho real dos outliers inseridos e média da distribuição a posteriori da probabilidade de uma observação ser outlier. São apresentados os resultados para as observações detectadas com outliers identificados (Prob. a posteriori i 0.5.) . . . . . . . . . . . . . 47

3.3 Média e desvios das distribuições a posteriori dos parâmetros do modelo para Ibovespa. . . . . . . . . . . . . . . . . . . . . . . . . . . 50

3.4 Outliers identificados no Ibovespa. . . . . . . . . . . . . . . . . . . . . 52

3.5 Média e desvios das distribuições a posteriori dos parâmetros do modelo para NYSE. . . . . . . . . . . . . . . . . . . . 55

3.6 Outliers identificados na NYSE e suas respectivas média posteriori da probabilidade de ser um outlier para observações com probabilidade a posteriori maior que $0.5 \ldots \ldots \ldots \ldots \ldots$. . . . . . . . . . 57

4.1 Valores para o conjunto de parâmetros das séries. . . . . . . . . . . . 70

4.2 Valores críticos para os modelos para $n=1000$ e nível de significância $5 \%$ baseados em 3000 replicações. . . . . . . . . . . . . . . . . . 72

4.3 Tamanho real do teste em porcentagem, quando o valor crítico é estimado utilizando parâmetros diferentes dos verdadeiros. . . . . . . . . 73

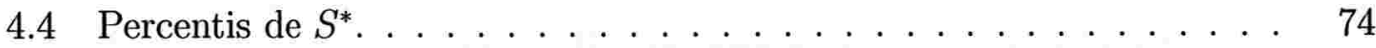


4.5 Valores influentes identificados no Ibovespa: Data de ocorrência, posição, retorno e valor da estatística. Valor crítico ao n.s. 5\% igual a 0.064. . 83

4.6 Valores ifluentes identificados na NYSE. . . . . . . . . . . . . 88

5.1 Estimativas dos percentis da máxima curvatura e inclinação. . . . . . 119

5.2 Propriedades distribucionais do maior componente do vetor diagnóstico significante classificados na Tabela 5.1 . . . . . . . . . . . . . . 120

5.3 Comparação das propriedades distribucionais para os modelos estimados para as séries do Ibovespa e NYSE dos maiores componentes do vetor diagnóstico significante classificados na Tabela 5.1. . . . . . . . 121

5.4 Percentis dos maiores componentes dos vetores diagnóstico da máxima curvatura e inclinação. . . . . . . . . . . . . . . . . . . . . 122

5.5 Diagnóstico baseado na curvatura e inclinação para Ibovespa. . . . . . 125

5.6 Diagnóstico baseado na curvatura e inclinação para NYSE. . . . . . 130

6.1 Comparação de métodos para série do Ibovespa. . . . . . . . . . . . 136

6.2 Comparação de métodos para série do NYSE. . . . . . . . . . . . . 137

6.3 Comparação da quantidade de valores detectados para série do NYSE. 138

6.4 Comparação da quantidade de valores detectados para série do Ibovespa.138 



\section{LISTA DE FIGURAS}

2.1 Série exemplo do conjunto de parâmetro $\Psi_{1}$ : Gráfico dos retornos e histograma. . . . . . . . . . . . . . . 13

2.2 Série exemplo do conjunto de parâmetro $\Psi_{2}$ : Gráfico dos retornos e histograma. . . . . . . . . . . . . . . . . . 14

2.3 Série exemplo para conjunto de parâmetro $\Psi_{3} \ldots \ldots \ldots$. . . . . . . 17

2.4 Gráfico dos retornos e histograma do Ibovespa. . . . . . . . . . . . . . 20

2.5 Gráfico dos retornos e histograma da NYSE. . . . . . . . . . . . . . . 22

3.1 Gráficos dos valores amostrados, histogramas das densidades posterioris dos parâmetros e correlograma para amostragem da série simulada sem outliers, $\Psi_{1} . \ldots \ldots \ldots \ldots \ldots$. . . . . . . . . . . . . 41

3.2 a) Retornos. b) Média da distribuição posteriori marginal do passo 1 para detecção de outliers da série simulada $\Psi_{1} \ldots \ldots \ldots \ldots$. . . . . . 42

3.3 Gráficos dos valores amostrados, histogramas das densidades posterioris dos parâmetros e correlograma para amostragem dos parâmetros da série simulada, com outliers, $\Psi_{3} \ldots \ldots \ldots \ldots \ldots$ 
3.4 Detecção de blocos de outliers da série simulada $\Psi_{3} 500$ primeiras observações. a) Retornos. b) Média da distribuição posteriori marginal

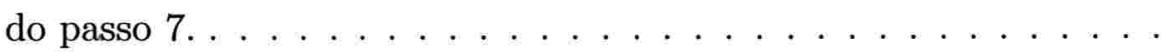

3.5 Detecção de blocos de outliers da série simulada $\Psi_{3} 500$ observações finais. a) Retornos. b) Média da distribuição posteriori marginal do

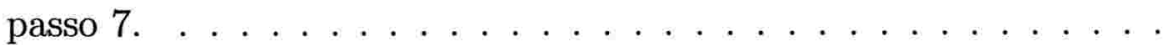

3.6 Gráfico do tamanho dos outliers detectados na série simulada $\Psi_{2}$ : a,b) Amostra da distribuição a posteriori. c,d) Histograma das distribuições a posteriori dos tamanhos dos outliers. . . . . . . . . . . . . . 48

3.7 Gráfico do tamanho dos outliers detectados na série simulada $\Psi_{2}$ : a,b) Amostra da distribuição a posteriori. c,d) Histograma das distribuições a posteriori dos tamanhos dos outliers. . . . . . . . . .

3.8 Gráficos dos valores amostrados, histogramas das densidades posterioris dos parâmetros e correlograma para amostragem da série simulada da série Ibovespa. . . . . . . . . . . . . . . . . .

3.9 Outliers detectados na série Ibovespa: a) Retornos. b) Média da distribuição a posteriori do passo 7 (dentro dos blocos) e ppasso 1 (fora dos blocos) para detecção de outliers. . . . . . . . . . . . . . . . . . 50

3.10 Outliers detectados na série Ibovespa: a) Retornos. b) Média da distribuição a posteriori do passo 7 (dentro dos blocos) e passo 1 (fora dos blocos) para detecção de outliers. . . . . . . . . . . . . . . . . 5

3.11 Outliers detectados na série Ibovespa: a) Retornos. b) Média da distribuição a posteriori do passo 7 (dentro dos blocos) e passo 1 (fora dos blocos) para deteç̧ão de outliers. . . . . . . . . . . . . 
3.12 Gráficos dos valores amostrados, histogramas das densidades posterioris dos parâmentros e correlograma para amostragem da série da NYSE. 54

3.13 Outliers detectados na série NYSE primeiras 500 observações: a) Retornos. b) Média da distribuição a posteriori do passo 7 (dentro dos blocos) e passo 1 (fora dos blocos) para deteç̧ão de outliers. . . . . . 55

3.14 Outliers detectados na série NYSE entre observação 501 e 1000: a) Retornos. b) Média da distribuição a posteriori do passo 7 (dentro dos blocos) e passo 1 (fora dos blocos) para detecção de outliers. . . . . . 56

3.15 Outliers detectados na série NYSE entre observação 1000 e 1255: a) Retornos. b) Média da distribuição a posteriori do passo 7 (dentro dos blocos) e passo 1 (fora dos blocos) para detecção de outliers. . . . . . 5

4.1 Histograma da distribuição dos máximos, $S^{*}$, baseada em 3000 replicações, tamanho da série igual a 1000 e conjunto de parâmetros

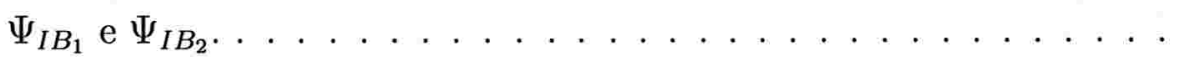

4.2 Histograma da distribuição dos máximos baseada em 3000 replicações, tamanho da série igual a 1000 e conjunto de parâmetros $\Psi_{N Y_{1}}$ e $\Psi_{N Y_{2}}$.

4.3 Distribuição empírica dos máximos para os modelos $\Psi_{I B_{1}}$ e $\Psi_{I B_{2}}$ para

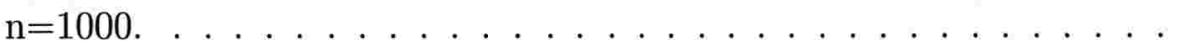

4.4 Distribuição empírica dos máximos para os modelos $\Psi_{N Y_{1}}$ e $\Psi_{N Y_{2}}$ para

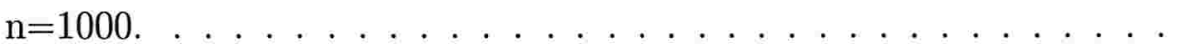

4.5 Série exemplo para conjunto de parâmetro $\Psi_{1}$ com valor crítico ao n.s. $5 \%$ igual a $0.0647 \ldots \ldots \ldots \ldots \ldots \ldots \ldots$

4.6 Série exemplo para conjunto de parâmetro $\Psi_{2}$ com valor crítico ao n.s. $5 \%$ igual a 0.072 . 
xviii

4.7 Série exemplo para conjunto de parâmetro $\Psi_{3}$ com valor crítico de $5 \%$. 78

4.8 Gráfico para análise de influência do Ibovespa: a)Retornos. b) Estatística do teste com valor de corte para n.s. 5\% da Tabela 4.2. c) Observações detectadas como influentes. . . . . . . . . . . . 79

4.9 Gráfico das 750 observações iniciais para análise de influência do Ibovespa: a)Retornos. b) Estatística do teste com valor de corte para n.s. $5 \%$ da Tabela 4.2. c) Observações detectadas como influentes. . . . . 80

4.10 Gráfico das 750 observações finais dos retornos e influência do Ibovespa: a)Retornos. b) Estatística do teste com valor de corte para n.s. 5\% da Tabela 4.2. c) Observações detectadas como influentes. . . . . . . . . 8

4.11 Gráfico das observações entre a posição 620 e 720 para análise de influência do Ibovespa: a)Retornos. b) Estatística do teste com valor de corte para n.s. $5 \%$ da Tabela 4.2. c) Observações como influentes. . .

4.12 Gráfico das observações entre a posição 900 e 950 para análise de influência do Ibovespa: a)Retornos. b) Estatística do teste com valor de corte para n.s. $5 \%$ da Tabela 4.2. c) Observações detectadas como influentes. . . . . . . . . . . . . .

4.13 Gráfico para análise de influência do NYSE: a)Retornos. b) Estatística do teste com valor de corte para n.s. $5 \%$ da Tabela 4.2. c) Observações detectadas como influentes. . . . . . . . . . . . . . . 8

4.14 Gráfico das 650 observações iniciais para Análise de Influência do NYSE: a)Retornos. b) Estatística do teste com valor de corte para n.s. 5\% interpolado dos valores da Tabela 4.2. c) Observações detectadas como influentes. . . . . . . . . . . . . . . 
4.15 Gráfico das 605 observações finais para análise de influência do NYSE: a)Retornos. b) Estatística do teste com valor de corte para n.s. 5\% interpolado dos valores da Tabela 4.2. c)Observações detectadas como influentes. . . . . . . . . . . . . . . . . . 86

4.16 Gráfico das observações entre a posição 150 e 250 para análise de influência do NYSE: a)Retornos. b) Estatística do teste com valor de corte para n.s. $5 \%$ interpolado dos valores da Tabela 4.2. c) Observações detectadas como influentes. . . . . . . . . . . . . . 86

4.17 Gráfico das observações entre a posição 400 e 500 para análise de influência do NYSE: a)Retornos. b) Estatística do teste com valor de corte para n.s. $5 \%$ interpolado dos valores da Tabela 4.2 . c) Observações detectadas como influentes. . . . . . . . . . . . . . . .

4.18 Gráfico das observações entre a posição 800 e 900 para análise de influência do NYSE: a)Retornos. b) Estatística do teste com valor de corte para n.s. $5 \%$ interpolado dos valores da Tabela 4.2 . c) Observações detectadas como influentes. . . . . . . . . . . . . . . . . 87

5.1 Ilustração do desvio de verossimilhança. . . . . . . . . . . . . . . . 95

5.2 Gráfico dos retornos e histograma do Ibovespa. . . . . . . . . . . . . . 123

5.3 Gráfico dos vetores diagnóstico para curvatura do Ibovespa . . . . . . 124

5.4 Gráfico dos vetores diagnóstico para inclinação do Ibovespa . . . . . . 124

5.5 Gráfico da série e retornos da NYSE. . . . . . . . . . . . . . . . . . . 127

5.6 Gráfico dos vetores diagnóstico para curvatura da NYSE . . . . . . 128

5.7 Gráfico dos vetores diagnóstico para inclinação do NYSE . . . . . . . 128 



\section{CAPÍTULO 1}

\section{Introdução}

A medida de volatilidade desempenha um papel fundamental em finanças e em economia de finanças. Por exemplo, ela é crucial no modelo média-variância de otimização de carteiras de investimento, como instrumento na análise de risco e na precificação de ativos e derivativos. Em todos estes casos são necessárias estimativas e previsões adequadas da volatilidade. Tais problemas são dificultados pelo fato das séries normalmente apresentarem observações extremas, bem distantes da distribuição da grande maioria das observações. Constatada empiricamente que as séries financeiras possuem cauda pesada alguns autores propuseram inicialmente a utilização de distribuições incondicionais com estas características. Algumas das distribuições incondicionais propostas foram as distribuições de Pareto estáveis, distribuição t-Student, distribuição de erros generalizados e misturas de distribuições. Outra alternativa foi incorporar ao processo uma dinâmica que explicasse esta característica. Nesse caso, dois modelos que obtiveram muito sucesso foram o modelo ARCH (ver por exemplo Engle, 1982) e de volatilidade estocástica (ver por exemplo Taylor, 1986 ). O primeiro modelo foi modificado de várias formas dando origem à família dos modelos XARCH (GARCH, 
EGARCH, QGARCH, SWGARCH, etc.). O segundo modelo também tem sido extensivamente estudado, mas é menos utilizado pelo fato da verossimilhança ser mais facilmente calculada na família dos modelos XARCH. Este problema foi diminuído um pouco com a proposta da utilização do método de quase-verossimilhança feita por Harvey et al. (1994). Posteriormente Jacquier et al. (1994) propuseram a utilização de técnicas Bayesianas onde não é necessário utilizar a verossimilhança aproximada. Nos últimos anos há um grande desenvolvimento na aplicação destes modelos com a utilização de métodos numéricos aproveitando o fato de que o modelo pode ser colocado na forma de espaço de estados com perturbações não Gaussianas na abordagem frequentista, ou na forma de modelos dinâmicos generalizados na abordagem Bayesiana (ver Shephard e Pitt, 1997 e Kim et al., 1998). Ao se colocar o modelo na forma dinâmica podemos também estudar o problema do ponto de vista Bayesiano com a utilização de uma estrutura probabilística para a ocorrência dos valores aberrantes.

Como estas observações chamadas de valores aberrantes ou outliers podem ter um efeito bastante negativo na especificação do modelo e na estimação dos parâmetros e das volatilidades, algumas abordagens têm sido propostas na literatura. Considerando a abordagem frequentista, Hotta e Tsay (1998) analisaram valores espúrios nos modelos GARCH e apresentaram um procedimento sequencial onde adicionaram parâmetros ao modelo e, através do teste de Multiplicador de Lagrange (LM), verificaram as significâncias dos parâmetros adicionados. Van Dijk et al. (1999) verificaram as propriedades do teste LM para os processos ARCH e GARCH na presença de outliers aditivos e observaram que eles têm influência no tamanho e poder do teste.

Considerando ainda a abordagem clássica, pesquisadores têm desenvolvido diversos trabalhos considerando a análise de influência como uma técnica para auxiliar na 
deteç̧ão de valores espúrios em modelos estatísticos. O artigo de Cook (1986) foi precursor da análise de influência utilizando o conceito de curvatura da geometria diferencial. Em artigos recentes Zhang (2004) e Zhang e King (2005) propuseram análise de influência em processos heteroscedásticos. Os autores estenderam o conceito inicialmente aplicado a modelos de regressão e analisaram modelos da família $\mathrm{ARCH}$, primeiro sob o aspecto de influência local em estatísticas de teste (Zhang, 2004) e posteriormente em diagnóstico para modelos GARCH (Zhang e King, 2005).

Em trabalho recente, Peña (2005) apresentou uma nova estatística para a deteç̧ão de valores influentes em modelo de regressão linear. Este resultado é ligeiramente simples de ser implementado e mostrou-se eficaz na detecção de valores espúrios que aparecem isolados ou conjuntamente. Além da eficácia do procedimento proposto verificada no artigo, Peña apresenta três propriedades teóricas da estatística proposta.

Sob a abordagem bayesiana McColloch e Tsay (1994) e Barnett et al. $(1996,1997)$ usaram MCMC para detectar outliers e obter distribuições posterioris dos parâmetros no modelo ARIMA. McColloch e Tsay (1994) verificaram que o amostrador de Gibbs é eficiente em estimar os parâmetros e detectar valores espúrios em processos Autoregressivo (AR), quando os outliers não ocorrem em conjunto. Justel et al. (2001) aperfeiçoaram o método proposto por McCulloch e Tsay (1994), adicionando um passo no algoritmo proposto inicialmente por eles. A evolução se deu através do acréscimo da amostragem das distribuições conjunta dos outliers.

Nesta tese serão apresentadas três propostas para a caracterização de valores influentes. A primeira proposta estende o tratamento de blocos de outliers em modelos Autoregressivos ao modelo de volatilidade estocástica, a segunda apresenta a análise de influência em modelos de volatilidade. Por último, a metodologia para análise de 
influência local é estendida ao modelo em estudo.

Este trabalho é organizado como segue: No capítulo 2 é apresentado o modelo de volatilidade estocástica, as extensões necessárias para acomodar a presença de valores espúrios e as séries simuladas e reais utilizadas nas análises no decorrer do trabalho. No capítulo 3 é considerada a abordagem Bayesiana para detecção de valores aberrantes em séries financeiras quando esses aparecem isolados e conjuntamente. No capítulo 4, é descrito o tratamento de análise de influência em modelos de volatilidades estocástica através de uma estatística de influência. O capítulo 5 apresenta um método de análise das séries financeiras através do conceito de influência local. Finalmente no capítulo 6 apresentamos as conclusões do trabalho desenvolvido. 


\section{CAPÍTULO 2}

\section{O Modelo de VE com Outliers}

Neste capítulo será apresentado o modelo de Volatilidade Estocástica considerado neste trabalho. Como trataremos as abordagens clássica e bayesiana para a análise de valores atípicos, o modelo é apresentado considerando-se os dois enfoques. A seção 2.1 apresenta a forma canônica do modelo de volatilidade estocástica e as modificações necessárias para a inclusão de outliers quando consideramos a análise com abordagem clássica. $\mathrm{Na}$ abordagem bayesiana, apresentamos as representações e as distribuições à priori para os parâmetros envolvidos. A seção 2.2 apresenta as séries simuladas que serão objetos de análise no decorrer no trabalho. Finalmente na seção 2.3 apresentamos as séries do Ibovespa e NYSE que serão analisadas através dos métodos propostos. 


\subsection{Modelo de Volatilidade Estocástica}

Os modelos mais utilizados para modelar a volatilidade são os modelos $\operatorname{GARCH}(\mathrm{p}, \mathrm{q})$ (ver Bollerslev et al., 1992) e o modelo de volatilidade estocástica (ver Ghysels et al., 1996 e Shephard, 1995). A série de retornos, $y_{t}$, é modelada como sendo um nível, $\mu_{t}$, mais uma perturbação, $\epsilon_{t}$, modelada pelo modelo de volatilidade. Tanto o modelo $\operatorname{GARCH}(\mathrm{p}, \mathrm{q})$ como o modelo de volatilidade condicional consideram que a perturbação é o resultante do produto de dois processos. De maneira formal temos:

$$
\begin{aligned}
y_{t} & =\mu_{t}+\epsilon_{t} \\
\epsilon_{t} & =\eta_{t} \sigma_{t},
\end{aligned}
$$

com as suposições de que:

- as perturbações $\left(\eta_{t}, \epsilon_{t}\right)$ e o nível $\left(\mu_{t}\right)$ são processos independentes;

- $\eta_{t}$ é um processo ruído branco com média zero e variância 1.

Com estas suposições temos que, condicionada a $\sigma_{t}$, a variância de $\epsilon_{t}$ é dada por $\sigma_{t}^{2}$.

O modelo de volatilidade estocástica é uma alternativa aos modelos ARCH propostos por Engle (1982) e suas várias extensões. A diferença básica entre esses modelos e o modelo de volatilidade estocástica, é que os modelos $\mathrm{ARCH}$ consideram a volatilidade como uma variável observável pois, sob certas condições, ela é conhecida quando os parâmetros do modelo e a série de retornos são conhecidos.

O modelo geral de volatilidade estocástica formulado inicialmente por Taylor (1986) considera, $\mathbf{y}=\left(y_{1}, \ldots, y_{T}\right)^{\prime}$ como um vetor gerado por um modelo de probabilidade $p(\mathbf{y} \mid \mathbf{h})$, onde $\mathbf{h}$ é um vetor de volatilidades e a variância $h_{t}$ evolui temporalmente, independente dos valores passados de $y_{t}$. As volatilidades são não observáveis 
e assume-se que são geradas por um mecanismo de probabilidade, $p(\mathbf{h} \mid \theta)$. A definição do modelo é dada a seguir.

Definição 2.1.1. Um processo y $y_{t}$ é chamado processo de volatilidade estocástica se

$$
y_{t}=\sigma_{t} \varepsilon_{t}
$$

onde $\varepsilon_{t}$ é um processo estacionário, e $\sigma_{t}$, a volatilidade condicional de $y_{t}$ no tempo $t$, é escrita como

$$
\sigma_{t}=e^{\frac{1}{2} h_{t}}
$$

onde $h_{t}$ segue um processo que pode ou não ser estacionário ${ }^{1}$. O termo $h_{t}$ é dito ser a log-volatilidade de $y_{t}$ e independe dos valores passados de $y_{t}$.

Na formulação mais simples $h_{t}$ é modelado como um processo autoregressivo de primeira ordem

$$
h_{t}=\alpha+\phi h_{t-1}+\eta_{t},
$$

onde $\alpha$ e $\phi$ são parâmetros invariantes no tempo com $|\phi|<1$ e $\eta_{t}$ é um processo estacionário com média zero e variância $\sigma_{\eta}^{2}$.

$\mathrm{Na}$ literatura pode-se encontrar o modelo de volatilidade estocástica representado de muitas maneiras. Serão apresentadas as formas que utilizaremos neste trabalho, ou seja, uma primeira forma de representação do modelo quando considerarmos a abordagem clássica e uma segunda forma ao utilizarmos o enfoque bayesiano.

\footnotetext{
${ }^{1}$ Em nosso caso trabalharemos com processo estacionário.
} 


\subsubsection{Modelo Clássico}

No capítulo 4 e capítulo 5 estaremos tratando da análise de influência e influência local sob o modelo de volatilidade. Nesses capítulos estaremos considerando a abordagem clássica do modelo, onde na estimação dos parâmetros e volatilidades a verossimilhança e as volatilidades são obtidas através do filtro de Kalman e/ou por métodos núméricos baseados em amostragem por importância. Inicialmente, apresentamos o modelo na forma canônica e a linearização do modelo necessária para a utilização do filtro de Kalman na obtenção da verossimilhança. Em seguida, a extensão para acomodar outliers é descrita.

O modelo de volatilidade estocástica na forma canônica, ou seja, o modelo das equações (2.1.3), (2.1.4) e (2.1.5) é dado por

$$
\begin{aligned}
y_{t} & =\beta e^{\frac{h_{t}}{2}} \varepsilon_{t}, \quad t \geqq 1, \\
h_{t+1} & =\mu+\phi\left(h_{t}-\mu\right)+\sigma_{\eta} \eta_{t}, \\
h_{t} & \sim N\left(\mu, \frac{\sigma_{\eta}^{2}}{1-\phi^{2}}\right),
\end{aligned}
$$

onde, fixa-se $\beta=1$ ou $\mu=0$ por problemas de identificabilidade e as outras suposições seguem como anteriormente. Em alguns capítulos será utilizada a formulação com $\beta=1$ e outros com $\mu=0$. Neste capítulo utilizaremos $\beta=1$. A fim de utilizar o filtro de Kalman devemos obter a linearidade da equação acima. Para tal tomamos uma transformação logaritmo do quadrado:

$$
\log \left(y_{t}^{2}\right)=h_{t}+\log \left(\varepsilon_{t}^{2}\right)
$$

Considerando que $\varepsilon_{t}$ é normalmente distribuído com $E\left[\varepsilon_{t}\right]=0$ e $\operatorname{Var}\left[\varepsilon_{t}\right]=1$ então 
$\log \left(\varepsilon_{t}^{2}\right)$ segue a distribuição log-qui-quadrado com média -1.27 e variância $\pi^{2} / 2$.

Considerando $\xi_{t}=\log \left(\varepsilon_{t}^{2}\right)-E\left[\log \left(\varepsilon_{t}^{2}\right)\right]=\log \left(\varepsilon_{t}^{2}\right)+1.27$, as equações em (5.3.1) serão modificadas para:

$$
\begin{array}{ll}
\log \left(y_{t}^{2}\right)=-1.27+h_{t}+\xi_{t}, & \xi_{t} \sim \operatorname{ID}\left(0, \frac{\pi^{2}}{2}\right), \\
h_{t}=\mu+\phi\left(h_{t-1}-\mu\right)+\eta_{t}, & \eta_{t} \sim \operatorname{NID}\left(0, \sigma_{\eta}^{2}\right)
\end{array}
$$

que está na forma de espaço de estados. Considerando as perturbações da equação de observação gaussiana podemos utilizar o filtro de Kalman para calcular a quasiverossimilhança e obter as estimativas de máxima quasi-verossimulhança (ver Harvey, et al. , 1994).

A fim de modelar uma série financeira sob a presença de valores atípicos, são apresentadas as extensões do modelo de volatilidade estocástica para dois tipos de outliers na série observada. Considera-se a ocorrência de valores aberrantes de nível ou aditivo (AO) que afeta o retorno apenas no tempo $t$ de ocorrência e outlier de volatilidade ou inovação (IO) cujo efeito é propagado na série.

Blocos(patches) de outliers têm sido objeto de estudo de muitos pesquisadores, onde podemos citar dentre outros os trabalhos de Chang e Liu (1993), Sánchez e Peña (1997) e Justel et al. (2001). Portanto, além de avaliarmos os métodos propostos quando os AOs ocorrem isoladamente, também estaremos avaliando quando eles ocorrem em blocos, ou seja, próximos.

Algumas representações das extensões do modelo, incluindo efeitos calendários e outliers podem ser encontrados em Fukui (2000). As ocorrências de um AO na $t_{\mathrm{AO}^{-}}$ ésima observação e de um IO na $t_{\mathrm{IO}}$-ésima observação, são incorporadas ao modelo 
da seguinte forma:

$$
\begin{aligned}
& \log \left(y_{t}^{2}\right)=-1.27+h_{t}+\xi_{t}+\Delta_{t}, \\
& \text { onde } \Delta_{t}=0 \mathrm{p} / t \neq t_{\mathrm{AO}} \text { e } \Delta_{t} \neq 0 \mathrm{p} / t=t_{\mathrm{AO}} \\
& h_{t}=\alpha+\phi h_{t-1}+\eta_{t}+\Lambda_{t}, \\
& \quad \text { onde } \Lambda_{t}=0 \mathrm{p} / t \neq t_{\mathrm{IO}} \text { e } \Lambda_{t} \neq 0 \mathrm{p} / t=t_{\mathrm{IO}} .
\end{aligned}
$$

Pode-se notar que a representação é similar à representação dos modelos dinâmicos apresentados por West e Harrison (1997). Entretanto, no enfoque bayesiano do modelo como um modelo hierárquico, teriam que ser consideradas as distribuições à priori que são apresentadas na próxima subseção.

\subsubsection{Modelo Bayesiano}

A representação do modelo de volatilidade com enfoque bayesiano pode ser descrita de forma análoga ao enfoque clássico, mas com as devidas alterações nas suposições. Dessa forma, podemos representar o modelo sem a acomodação de outlier da seguinte forma:

$$
\begin{aligned}
& y_{t}=\beta e^{\frac{h_{t}}{2}} \epsilon_{t}, \\
& h_{t}=\phi h_{t-1}+\sigma_{\eta} \eta_{t},
\end{aligned}
$$

onde, $\epsilon_{t}$ e $\eta_{t}$ são considerados como anteriormente e devemos completar o modelo considerando as densidades a priori para os modelos a serem estimados. Considerando todas as prioris independentes, para o parâmetro $\mu$ atribuiremos uma priori difusa $\mu \sim N(0,10)$, para $\sigma_{\eta}^{2} \mid \phi, \mu \sim \mathcal{I} G\left(\sigma_{r} / 2, S_{\sigma} / 2\right)$ (Gama inversa) onde $\sigma_{r}=5$ e $S_{\sigma}=$ $0.01 \times \sigma_{r}$. Para o parâmetro autoregressivo foi considerada a priori dada por:

$$
\pi(\phi) \propto\left\{\frac{(1+\phi)}{2}\right\}^{\phi^{(1)}-1}\left\{\frac{(1-\phi)}{2}\right\}^{\phi^{(2)}-1}, \quad \phi^{(1)}, \phi^{(2)}>\frac{1}{2} .
$$


Através dessa priori Kim et al. (1998) obtiveram suporte em $(-1,1)$. Foi fixado $\phi^{(1)}=20$ e $\phi^{(2)}=1.5$ obtendo-se uma média da priori de 0.86 .

Considerando o enfoque bayesiano, o efeito do AO será incorporado acrescentando-se algumas variáveis ao modelo. Teremos a localização $\delta_{t}$, e o tamanho $\beta_{t}$ do valor atípico, i.e., $\delta_{t}$ nos fornecerá a probabilidade de ocorrência de um outlier no tempo $t$. O modelo considerando outlier do tipo aditivo será dado por:

$$
\begin{aligned}
& y_{t}=\delta_{t} \beta_{t}+e^{\frac{h_{t}}{2}} \epsilon_{t}, \\
& h_{t}=\mu+\phi\left(h_{t-1}-\mu\right)+\sigma_{\eta} \eta_{t},
\end{aligned}
$$

onde $y_{t}$ são os retornos e os erros $\epsilon_{t}$ e $\eta_{t}$ possuem distribuição normal padrão. Neste modelo é considerado que $\delta_{t}$ tem distribuição a priori $\operatorname{Ber}(k)$, onde $k$ tem distribuição $\operatorname{Beta}\left(u_{0}, n_{0}\right)$ com $u_{0}$ e $n_{0}$ sendo os hiperparâmetros. O parâmetro, $\log \left(1+\beta_{t}\right)$, onde $\beta_{t}$ é o tamanho do outlier, possui distribuição a priori normal com média $-0.5 \nu^{2} \mathrm{e}$ variância $\nu^{2}$ e para $\nu$ é suposta uma distribuição log-normal com parâmetros $\left(\nu_{0}, V_{0}\right)$. Finalmente, a priori para $\sigma_{\eta}$ tem distribuição log-normal com parâmetros $\left(\sigma_{0}, \Sigma_{0}\right)$.

O modelo onde consideram-se valores atípicos do tipo IO será dado por:

$$
\begin{aligned}
& y_{t}=e^{\frac{h_{t}}{2}} \epsilon_{t}, \\
& h_{t}=\delta_{t} \beta_{t}+\mu+\phi\left(h_{t-1}-\mu\right)+\sigma_{\eta} \eta_{t},
\end{aligned}
$$

com as mesmas suposições do modelo anterior. 


\subsection{Séries Simuladas}

Apresentaremos nesta seção as séries simuladas utilizadas nas análises dos próximos capítulos. Inicialmente serão apresentadas algumas análises descritivas das séries simuladas sem outliers e mudanças estruturais. Posteriormente são introduzidos os dois tipos de outliers presentes na literatura, outliers aditivo e de inovação. Vale ressaltar que também estaremos considerando a presença de blocos de outliers aditivos.

\subsubsection{Série Sem Outliers e Mudanças Estruturais}

Nesta subseção serão apresentadas as séries simuladas sem a presença de outliers. Os parâmetros do modelo de volatilidade da equação (2.1.6), $\Psi=\left(\beta, \phi, \sigma_{\eta}^{2}\right)$, serão estimados através da quasi-verosimilhança a fim de diminuir o tempo computacional. Esse tempo seria bem maior se considerássemos as estimativas dos parâmetros pela verossimilhança exata. Os parâmetros $\beta$ e $\mu$ foram simulados com valores 1 e zero, respectivamente.

As estimativas dos parâmetros foram calculadas a partir da quasi-verossimilhança obtida através do pacote SSFPACK proposto por Koopman et al. (1999) e foi utilizado o método numérico BFGS (Broyden-Fletcher-Goldfarb-Shanno) para a maximização desta função.

Além disso, vale lembrar que para o valor inicial de $h_{0}$ foi tomada como a média dos valores estimados dos primeiros 100 valores de $h_{t}$. Foram consideradas as estimativas suavizadas calculadas através do filtro de Kalman. 
Foram geradas séries de tamanho 1000 considerando os seguintes conjuntos de parâmetros para o processo gerador de dados:

\begin{tabular}{||c||ccc||}
\hline \hline \multicolumn{1}{|c||}{} & \multicolumn{3}{c||}{ Parâmetros } \\
\hline Modelos & $\phi$ & $\sigma_{\eta}$ & $\beta$ \\
\hline$\Psi_{1}$ & 0.98 & 0.12 & 1.0 \\
$\Psi_{2}$ & 0.95 & 0.20 & 1.0 \\
\hline \hline
\end{tabular}

Tabela 2.1: Conjunto de parâmetros para o processo gerador de dados.

Os valores dos parâmetros foram escolhidos baseados em valores presentes na literatura dos modelos de VE. Os resultados das simulações serão analisados através de algumas estatísticas descritivas e as densidades serão estimadas por um método não-paramétrico considerado na linguagem $O X$.

A Figura (2.1) apresenta a simulação considerando o conjunto de parâmetros $\Psi_{1}$ e a Figura (2.2) os parâmetros $\Psi_{2}$. Na parte superior da figura podemos verificar a série simulada. Na parte inferior da figura, podemos avaliar algumas características da distribuição de uma série financeira a partir do histograma apresentado.

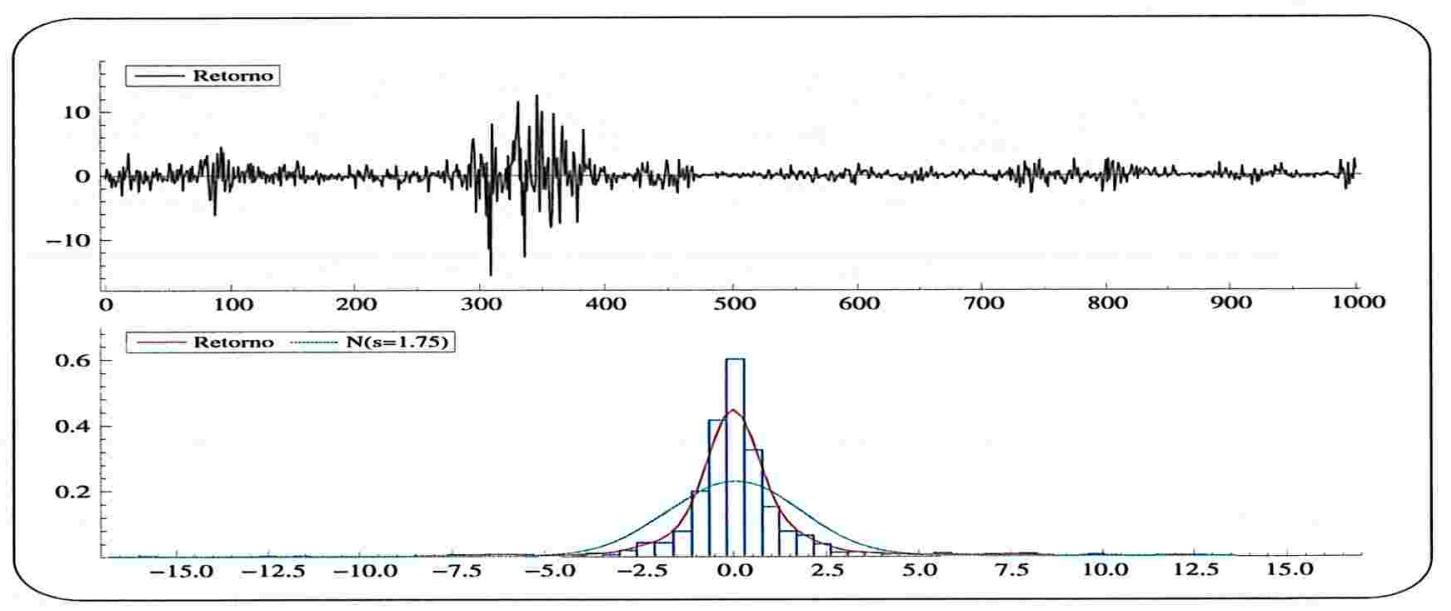

Figura 2.1: Série exemplo do conjunto de parâmetro $\Psi_{1}$ : Gráfico dos retornos e histograma. 


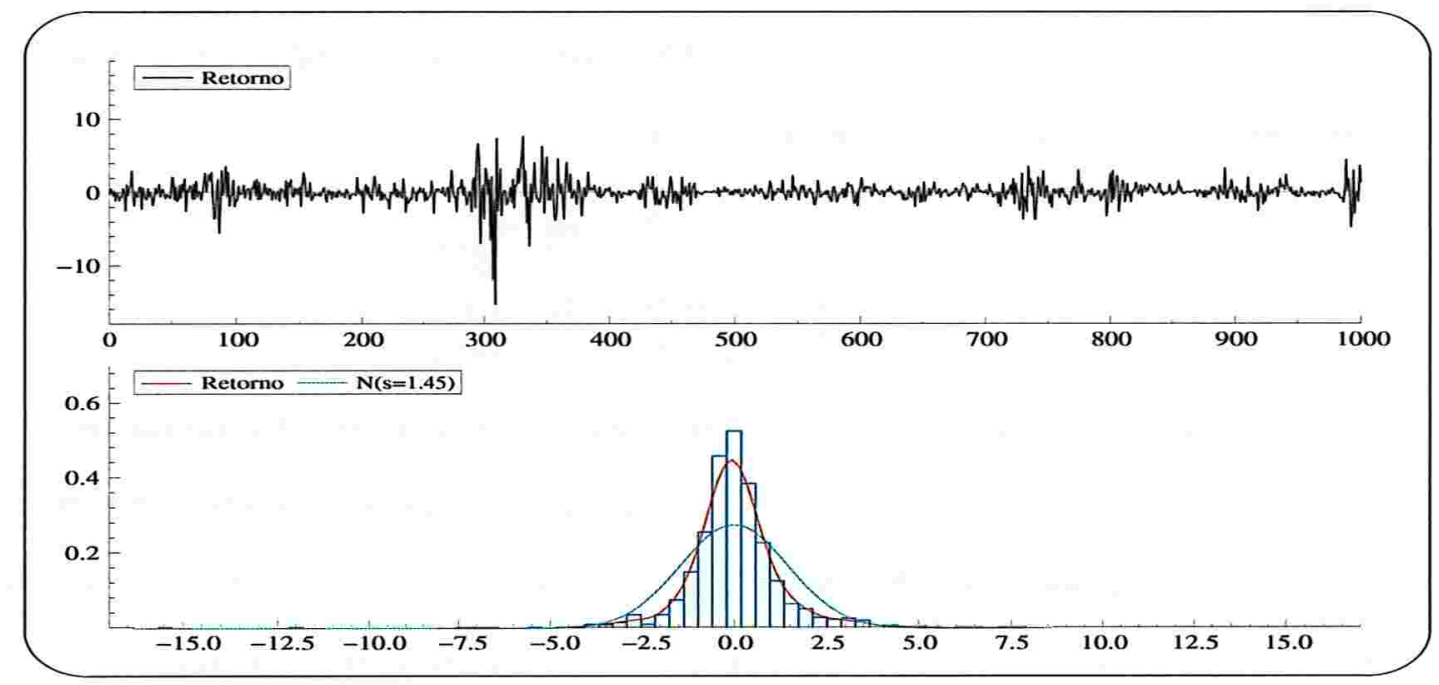

Figura 2.2: Série exemplo do conjunto de parâmetro $\Psi_{2}$ : Gráfico dos retornos e histograma.

A Tabela 2.2 apresenta as estatísticas descritivas para as séries simuladas. Podemos verificar as características distribucionais das séries financeiras através dos resultados obtidos.

\begin{tabular}{||c||c|c|c|c|c||}
\hline \hline Modelos & D. Padrão & Assimetria & Excesso Curtose & Mínimo & Máximo \\
\hline \hline$\Psi_{1}$ & 1.748300 & -0.213750 & 20.42800 & -15.62600 & 12.54700 \\
$\Psi_{2}$ & 1.453100 & -1.447500 & 20.60400 & -15.32200 & 7.675500 \\
\hline \hline
\end{tabular}

Tabela 2.2: Estatísticas descritivas das séries simuladas.

As Tabelas 2.3 e 2.3 apresentam as estimativas dos parâmetros e seus respectivos desvios para as séries simuladas com os conjuntos de parâmetros $\Psi_{1}$ e $\Psi_{2}$, respectivamente. Nas tabelas ilustramos as estimativas obtidas pelos três métodos utilizados nos capítulos posteriores. A primeira estimativa foi obtida pelo método bayesiano utilizado no Capítulo 3, a segunda por quasi-máxima verossimilhança utilizada no Capítulo 4 e a última utilizando a verossimilhança exata aplicada no Capítulo 5. 


\begin{tabular}{|c|c|c|c|c|}
\hline Parâmetros & $\begin{array}{c}\text { Estimativa } \\
\text { Bayesiana }\end{array}$ & $\begin{array}{c}\text { Quasi } \\
\text { Verossimilhança }\end{array}$ & $\begin{array}{c}\text { Verossimilhança } \\
\text { Exata }\end{array}$ & $\begin{array}{l}\text { Valor } \\
\text { Real }\end{array}$ \\
\hline$\phi$ & $\begin{array}{c}0.9746 \\
(\mathbf{0 . 0 0 8 1 )}\end{array}$ & $\begin{array}{c}0.9836 \\
(0.0105)\end{array}$ & $\begin{array}{c}0.9788 \\
(0.0089)\end{array}$ & 0.9800 \\
\hline$\sigma_{\eta}^{2}$ & $\begin{array}{c}0.0152 \\
(0.0093)\end{array}$ & $0_{(0.0103)}^{0.0161}$ & $\underbrace{0.0149}_{(0.0099)}$ & 0.0144 \\
\hline$\beta$ & $\begin{array}{l}1.0128 \\
(0.1339)\end{array}$ & $\begin{array}{r}0.9836 \\
(0.1527) \\
\end{array}$ & $\begin{array}{l}1.03255 \\
(0.1421)\end{array}$ & 1.0000 \\
\hline
\end{tabular}

Tabela 2.3: Estimativas dos parâmetros para série simulada com conjunto de parâmetros $\Psi_{1}$.

\begin{tabular}{|c|c|c|c|c|}
\hline Parâmetros & $\begin{array}{l}\text { Estimativa } \\
\text { Bayesiana }\end{array}$ & $\begin{array}{c}\text { Quasi } \\
\text { Verossimilhança }\end{array}$ & $\begin{array}{l}\text { Verossimilhança } \\
\text { Exata }\end{array}$ & $\begin{array}{l}\text { Valor } \\
\text { Real }\end{array}$ \\
\hline$\phi$ & 0.9513 & 0.9328 & 0.9528 & 0.9500 \\
\hline$\sigma_{\eta}^{2}$ & 0.0483 & 0.0365 & 0.0493 & 0.0400 \\
\hline$\beta$ & $\begin{array}{r}(0.0231) \\
(0.1372)\end{array}$ & $\begin{array}{r}0.9622 \\
(0.1451)\end{array}$ & $1_{(0.1629)}^{(0.018)}$ & 1.0000 \\
\hline
\end{tabular}

Tabela 2.4: Estimativas dos parâmetros para série simulada com conjunto de parâmetros $\Psi_{2}$.

Podemos verificar através das Tabelas 2.3 e 2.3 que os valores foram estimados próximos dos valores dos parâmetros considerados reais. 


\subsubsection{Série Com Outlier e Mudanças Estruturais}

As séries com outliers consideradas neste trabalho serão apresentadas nessa subseção. Foram consideradas a presença de dois outliers isolados do tipo aditivo, um bloco de 5 outliers consecutivos do tipo $\mathrm{AO}$ e outro IO isolado.

O tamanho dos AOs foi tomado como sendo igual a $\Delta$ desvios padrões da série de retornos simulada sem outlier e o IO foi tomado como sendo igual a $\Lambda$ vezes o desvio padrão do processo autoregressivo, i.e., $\Lambda\left[\sigma_{\eta}^{2}\left(1-\phi^{2}\right)^{-1}\right]^{0.5}$. Em todas as séries foram mantidos os mesmos conjuntos de perturbações nas equações de observação e estado. As séries analisadas foram geradas com os conjuntos de parâmetros dados na Tabela 2.5.

\begin{tabular}{||c|ccccccc||}
\hline \hline Modelos & $\phi$ & $\sigma_{\eta}$ & $\beta$ & $\Delta_{(50,0)}$ & $\Delta_{(250,5)}$ & $\Delta_{(500,0)}$ & $\Lambda_{700}$ \\
\hline$\Psi_{3}$ & 0.98 & 0.12 & 1.0 & 8 & 4 & 5 & 5 \\
$\Psi_{4}$ & 0.95 & 0.20 & 1.0 & 8 & 4 & 5 & 5 \\
\hline \hline
\end{tabular}

Tabela 2.5: Conjunto de parâmetros para o processo gerador de dados: $\Delta_{(t, \kappa)}$ é o tamanho dos outliers do tempo $t$ até o tempo $t+\kappa$.

Na Tabela 2.5 temos que $\Delta_{(t, \kappa)}$ é o tamanho dos outliers do tempo $t$ até o tempo $t+\kappa$, ou seja, $\kappa$ é o tamanho do bloco de valores aberrantes. O tamanho do outlier de inovação é dado por $\Lambda$.

Como pode ser verificado na Tabela 2.5 foram colocados um AO isolado em $t=50$ e $t=500$, um bloco de outliers de tamanho 5 em $t=250$ e um IO em $t=700$ nas séries em estudo.

A Figura 2.3 apresenta a série simulada com o conjunto de parâmetros $\Psi_{3}$ onde consideramos todas as configurações de outliers analisadas neste trabalho. 


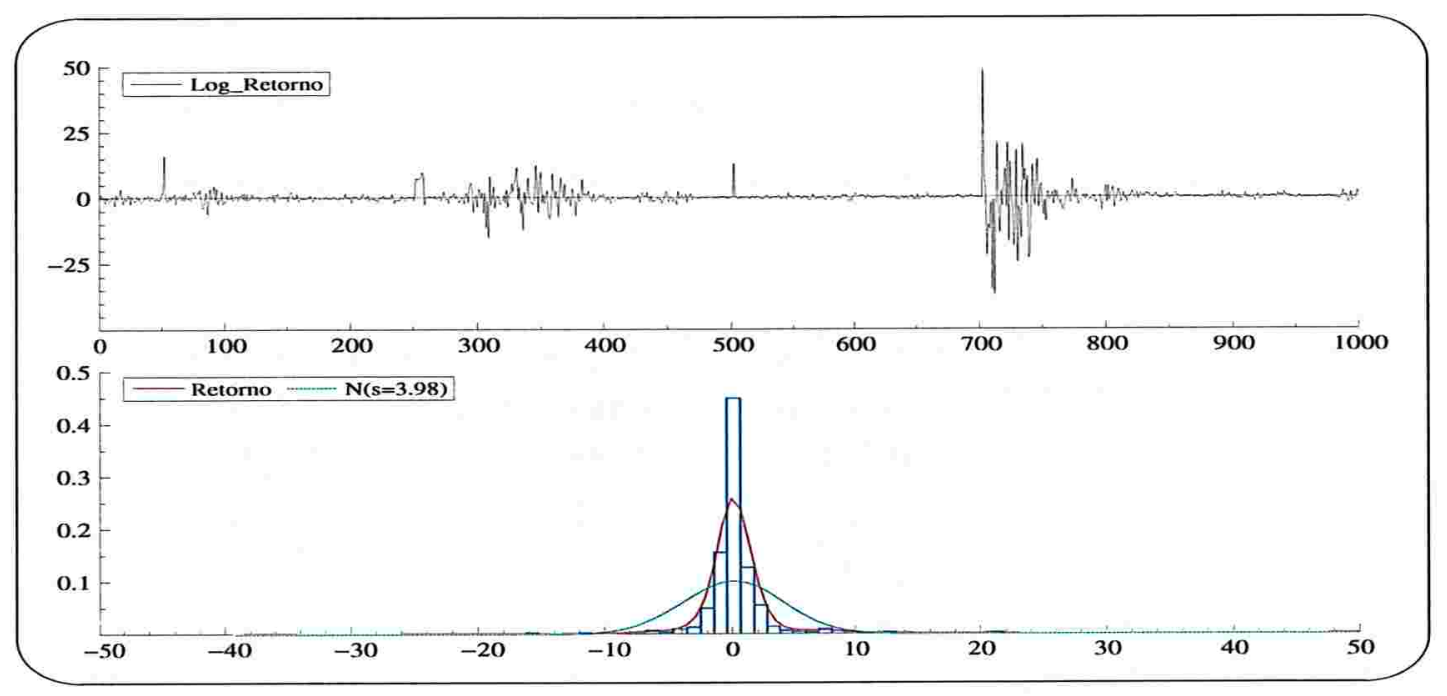

Figura 2.3: Série exemplo para conjunto de parâmetro $\Psi_{3}$.

A Tabela 2.6 apresenta algumas estatísticas descritivas dos casos onde consideramos a ocorrência conjunta dos tipos de valores espúrios e a ocorrência isolada. Pode-se notar que a presença de um outlier do tipo AO na série tem influência maior na média dos retornos, enquanto que a presença de um IO aumenta sensivelmente o desvio padrão da série e altera o sinal do coeficiente de assimetria.

\begin{tabular}{||c||c|c|c|c|c|c||}
\hline \hline Tipo Outlier & Média & D. Padrão & Assimetria & Excesso Curtose & Mínimo & Máximo \\
\hline \hline AO IO BL & 0.019504 & 3.9694 & -0.01334 & 45.043 & -37.336 & 48.888 \\
AO & 0.022543 & 1.8616 & 0.70650 & 22.654 & -15.652 & 16.086 \\
IO & 0.012430 & 3.8709 & -0.11228 & 49.640 & -37.265 & 48.958 \\
BL & 0.023706 & 1.8268 & 0.07358 & 17.969 & -15.664 & 12.509 \\
\hline \hline
\end{tabular}

Tabela 2.6: Estatísticas descritivas das séries simuladas com outliers. 
As Tabelas 2.7 e 2.8 apresentam as estimativas dos parâmetros e seus respectivos desvios padrões para as séries simuladas com os conjuntos de parâmetros $\Psi_{3}$ e $\Psi_{4}$, respectivemente. Nas tabelas ilustramos as estimativas obtidas pelos três métodos utilizados nos capítulos posteriores.

\begin{tabular}{c|ccc}
\hline \hline Parâmetros & $\begin{array}{c}\text { Estimativa } \\
\text { Bayesiana }\end{array}$ & $\begin{array}{c}\text { Quasi } \\
\text { Verossimilhança }\end{array}$ & $\begin{array}{c}\text { Verossimilhança } \\
\text { Exata }\end{array}$ \\
\hline$\phi$ & 0.9731 & 0.9668 & 0.9740 \\
$(0.0154)$ & $0.0178)$ & $0.0143)$ \\
$\sigma_{\eta}^{2}$ & 0.0183 & 0.0201 & 0.0191 \\
$\beta$ & $1_{(0.0315)}$ & $(0.01243$ & $1_{(0.0329)}$ \\
$\beta$ & $(0.0425$ & 1.0918 \\
\hline \hline
\end{tabular}

Tabela 2.7: Estimativas dos parâmetros para série simulada com conjunto de parâmetros $\Psi_{3}$.

\begin{tabular}{c|ccc}
\hline \hline Parâmetros & $\begin{array}{c}\text { Estimativa } \\
\text { Bayesiana }\end{array}$ & $\begin{array}{c}\text { Quasi } \\
\text { Verossimilhança }\end{array}$ & $\begin{array}{c}\text { Verossimilhança } \\
\text { Exata }\end{array}$ \\
\hline$\phi$ & 0.9305 & 0.9281 & 0.9329 \\
$(0.0098)$ & $0.0105)$ & $0.016)$ \\
$\sigma_{\eta}^{2}$ & 0.0273 & 0.0146 & 0.0198 \\
$\beta$ & $(0.0317)$ & $(0.0103)$ & $(0.0321)$ \\
$\beta$ & 1.0329 & 0.9529 & 1.0436 \\
$(0.2242)$ & $(0.1923)$ & $(0.2176)$ \\
\hline \hline
\end{tabular}

Tabela 2.8: Estimativas dos parâmetros para série simulada com conjunto de parâmetros $\Psi_{4}$. 


\subsection{Séries Empíricas}

Neste trabalho utilizamos duas séries de dados reais para aplicação das metodologias propostas. A primeira é a série do índice Bovespa analisada para um período de aproximadamente 5 anos, o segundo conjunto de dados é a série da NYSE, também analisada por Zhang e King (2005), em um período de 4 anos.

\subsubsection{Série do IBOVESPA}

Os retornos são do período de 03 de Janeiro de 1995 a 27 de Dezembro de 2000, num total de 1500 observações. Durante este período ocorreram várias crises em outros países que influenciaram o mercado financeiro brasileiro. As principais crises foram: crise do México em Fevereiro e Março de 1995, crise na Ásia em Outubro de 1997, moratória da Rússia em Agosto de 1998, crise do Brasil (desvalorização do real) em Janeiro de 1999 e Nasdaq em Abril de 2000. Os retornos corrigidos da série foram calculados da seguinte forma ${ }^{2}$ :

$$
y_{t}=100 \times\left\{\left(\log r_{t}-\log r_{t-1}\right)-n^{-1} \sum_{i=1}^{n}\left(\log r_{i}-\log r_{i-1}\right)\right\},
$$

onde $n$ é o número de observações. A Tabela 2.9 apresenta os valores das estatísticas descritivas dos retornos e o gráfico da Figura 5.2 permite observar alguns dos fatos estilizados existentes em séries financeiras.

\footnotetext{
${ }^{2}$ Seguindo sugestão dada por Kim et al. (1998).
} 


\begin{tabular}{||c|c||}
\hline Medidas & Ibovespa \\
\hline \hline D. Padrão & 2.859335 \\
Assimetria & 0.743485 \\
Excesso Curtose & 12.944355 \\
Mínimo & -17.316949 \\
Máximo & 28.729927 \\
\hline
\end{tabular}

Tabela 2.9: Estatísticas descritivas dos retornos do Ibovespa.

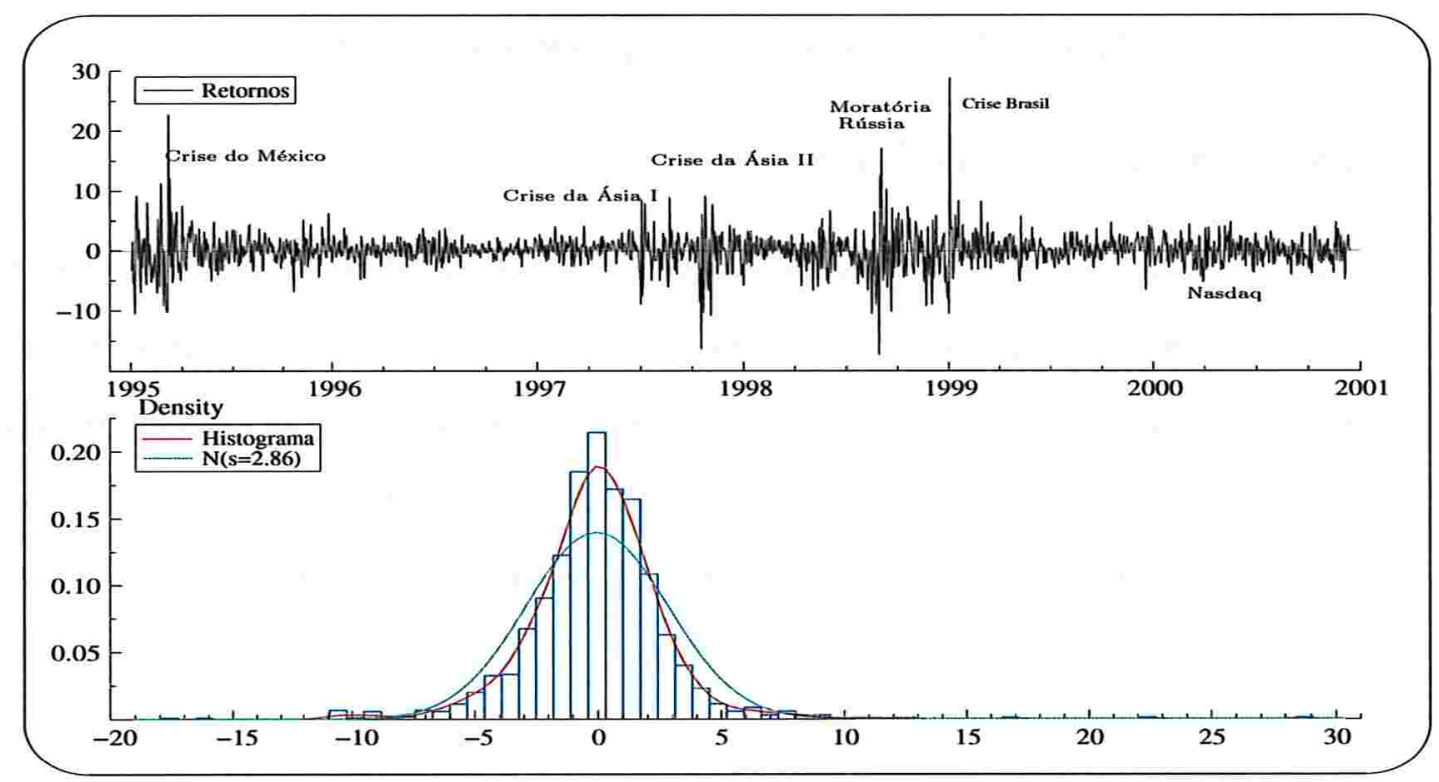

Figura 2.4: Gráfico dos retornos e histograma do Ibovespa.

Através do histograma da Figura 2.4, pode-se verificar que a da distibuição dos retornos possuem caudas mais pesadas do que a distribuição normal e alguns valores se encontram vários desvios padrões distantes da média. Os conglomerados de volatilidade podem ser verificados através do gráfico de retornos, onde os períodos de alta volatilidade coincidem com os períodos onde ocorreram as crises citadas no início da subseção. 
A Tabela 2.10 apresenta as estimativas dos parâmetros e seus respectivos desvios para a série do Ibovespa. Na tabela ilustramos as estimativas obtidas pelos três métodos utilizados nos capítulos posteriores.

\begin{tabular}{c|ccc}
\hline \hline Parâmetros & $\begin{array}{c}\text { Estimativa } \\
\text { Bayesiana }\end{array}$ & $\begin{array}{c}\text { Quasi } \\
\text { Verossimilhança }\end{array}$ & $\begin{array}{c}\text { Verossimilhança } \\
\text { Exata }\end{array}$ \\
\hline$\phi$ & 0.9719 & 0.9811 & 0.9733 \\
$\sigma_{\eta}^{2}$ & $0.0098)$ & $0.0112)$ & 0.01010 \\
$\beta$ & 0.0518 & 0.0417 & 0.0532 \\
$\beta$ & $2.0437)$ & $(0.0553)$ & $(0.0419)$ \\
$(0.2519)$ & 2.2336 & $\underset{(0.3147)}{(0.27414)}$ \\
\hline \hline
\end{tabular}

Tabela 2.10: Estimativas dos parâmetros para Ibovespa. 


\subsubsection{Série da NYSE}

A série de retornos da NYSE é considerada no período de 02 de Janeiro de 1997 a 31 de Dezembro de 2001 num total de 1255 observações. A Tabela 2.11 apresenta os valores das estatísticas descritivas dos retornos e o gráfico da Figura 2.5, permite observar o comportamento da série e alguns dos fatos estilizados.

\begin{tabular}{||c|c||}
\hline Medidas & NYSE \\
\hline \hline D. Padrão & 1.2446 \\
Assimetria & -0.55291 \\
Excesso Curtose & 3.8130 \\
Mínimo & -7.4864 \\
Máximo & 4.8193 \\
\hline
\end{tabular}

Tabela 2.11: Estatísticas descritivas dos retornos da NYSE.

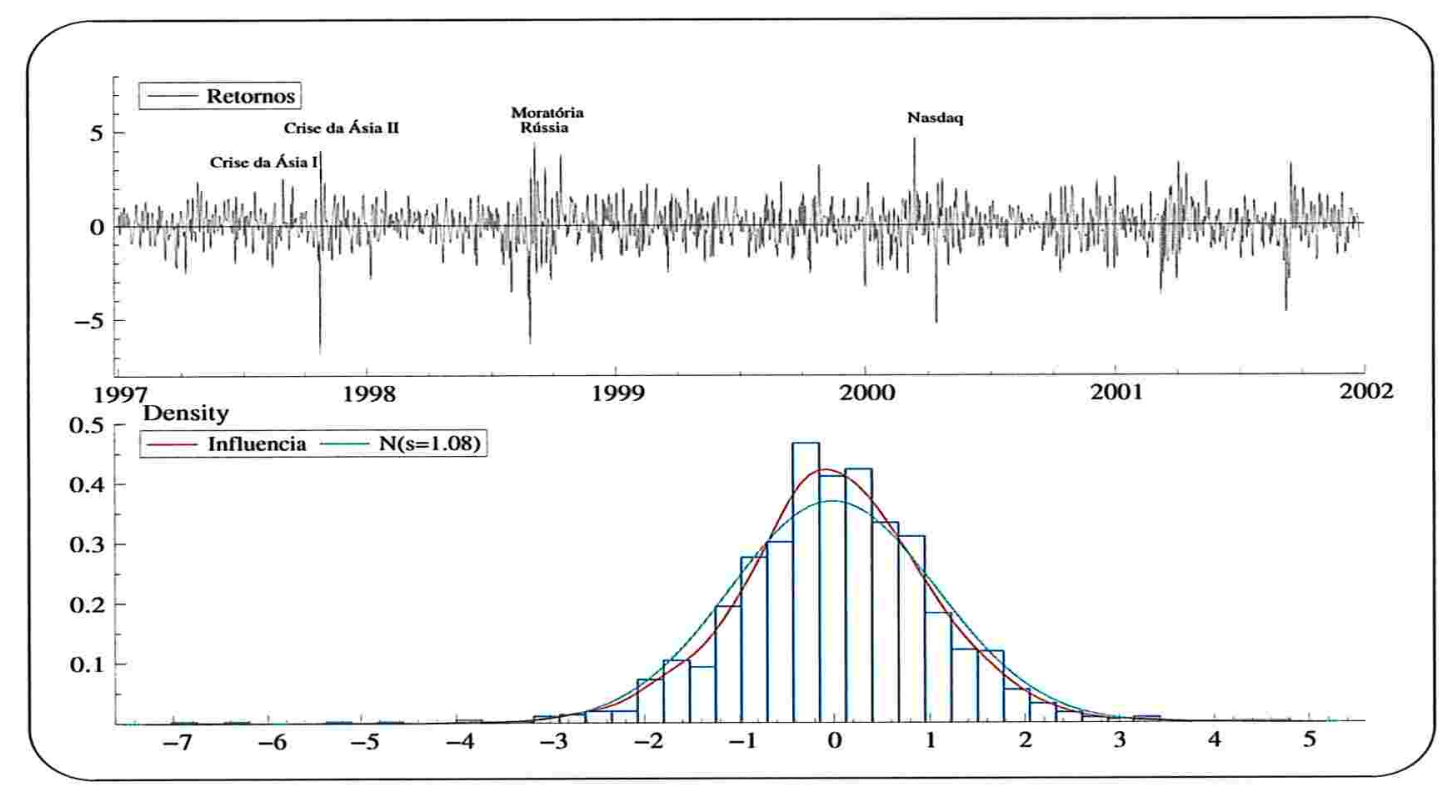

Figura 2.5: Gráfico dos retornos e histograma da NYSE.

Podemos verificar pela Figura 2.5 que durante este período ocorreram várias crises que influenciaram o mercado financeiro nos EUA. As principais crises foram: Crise 
na Ásia em Outubro de 1997, moratória da Rússia em Agosto de 1998 e Nasdaq em Abril de 2000 .

A Tabela 2.12 apresenta as estimativas dos parâmetros e seus respectivos desvios para a série do NYSE. Na tabela ilustramos as estimativas obtidas pelo três métodos utilizados nos capítulos posteriores.

\begin{tabular}{|c|c|c|c|}
\hline Parâmetros & $\begin{array}{l}\text { Estimativa } \\
\text { Bayesiana }\end{array}$ & $\begin{array}{c}\text { Quasi } \\
\text { Verossimilhança }\end{array}$ & $\begin{array}{l}\text { Verossimilhança } \\
\text { Exata }\end{array}$ \\
\hline$\phi$ & $\begin{array}{l}0.9398 \\
(0.0105)\end{array}$ & $\begin{array}{c}0.9310 \\
(0.0135)\end{array}$ & $\begin{array}{l}0.9352 \\
(0.0098)\end{array}$ \\
\hline$\sigma_{\eta}^{2}$ & $\begin{array}{l}0.0463 \\
(0.0101)\end{array}$ & 0.0328 & 0.0369 \\
\hline$\beta$ & 1.2052 & $\begin{array}{l}1.2236 \\
(0.1452)\end{array}$ & 1.2056 \\
\hline
\end{tabular}

Tabela 2.12: Estimativas dos parâmetros para NYSE.

As séries empíricas consideradas são objetos de estudos na literatura, Zhang e King (2005) analisam influência local na série da NYSE considerando os modelos GARCH. A série do Ibovespa foi analisada por vários pesquisadores, por exemplo, Motta (2001) e Fukui (2000), este último apresenta um procedimento de varredura para a deteç̧ão de outliers.

\subsection{Conclusões}

Neste capítulo apresentamos o modelo de volatilidade estocástica sob o enfoque bayesiano e freqüentista. Algumas formas de representação do modelo foram exploradas de forma a considerarmos valores atípicos. As séries de retornos simuladas que, serão analisadas durante este trabalho, são apresentadas e também algumas de suas propriedades. Apresentamos as séries de dados reais do Ibovespa e NYSE que serão objetos de estudos após o desenvolvimento da metodologia de cada capítulo. Em todos os 
modelos estimados as estimativas encontradas pelos três métodos foram bastante próximas 


\section{CAPÍTULO 3}

\section{Detecção de Blocos de Outliers em Modelos de Volatilidade Estocástica}

Entre os fatos estilizados das séries financeiras está a existência de outliers e sua freqüente ocorrência em blocos, isto é, outliers próximos uns dos outros. Isto é perfeitamento explicado pelo fato de que choques econômicos/políticos não necessariamente afetam o mercado em apenas um dia e podendo ter suas influências observadas ao longo de um curto período de tempo. Por outro lado, sabemos que nestes casos muitas das técnicas estatísticas desenvolvidas para deteção de outliers tem sua eficácia diminuída. Com o objetivo de minorar este problema Justel et al. (2001), baseados na abordagem bayesiana de McCulloch e Tsay (1994) apresentaram um procedimento para deteç̧ão e estimação de conjuntos de outliers para processos autoregressivos. Eles propuseram utilizar as distribuições posterioris de $\beta_{t}$ (tamanho do outlier) e de $\delta_{t}$ (função indicadora da presença de outlier na t-ésima observação) para detectar e estimar o tamanho do outlier. A metodologia é desenvolvida de forma a ser eficaz 
para detectar blocos de outliers.

Neste capítulo será estendido o algoritmo proposto por Justel et al. (2001) ao modelo de volatilidade estocástica. Serão considerados dois tipos de outliers e obtidas as densidades posteriores necessárias para estimativas dos parâmetros do modelo e o algoritmo de deteç̧ão de blocos de outliers. A primeira seção apresenta uma pequena introdução sobre a metodologia desenvolvida para deteção de outliers em modelos de séries temporais. O procedimento para deteção dos blocos de outliers é apresentado na seção 2 , enquanto a seção 3 apresenta suscintamente os algoritmos utilizados para estimação do modelo de volatilidade estocástica. Vale ressaltar que no decorrer do texto as médias das distribuições a posteriori em muitos casos são denominadas (com abuso de linguagem) de estimativas. Finalmente, será apresentada a análise de séries simuladas e a aplicação da metodologia às séries de retornos do Ibovespa e NYSE.

\subsection{Outliers em Modelos de Volatilidade}

Desde o artigo de Fox (1972), que introduziu alguns tipos de outliers em modelos de séries temporais, apareceram na literatura muitos procedimentos para tratar estes valores espúrios em séries temporais. Por exemplo, entre os métodos iterativos desenvolvidos para detectar perturbações em modelos ARIMA, podemos citar Chang, Tiao e Chen (1988) e Tsay $(1986,1988)$. No entanto, a maioria desses procedimentos não são eficazes quando considera-se blocos de outliers. Se por um lado alguns outliers não são detectados devido ao efeito de mascaramento, por outro lado, podemos classificar "boas" observações como outliers, efeito este chamado de swamping. Chen e Liu (1993) apresentaram um procedimento iterativo para reduzir o efeito de mascaramento, estimando conjuntamente os parâmetros e as magnitudes do efeito do 
outlier. Como tal procedimento começa com a estimativa do modelo desconsiderando a presença de valores espúrios nos dados, Sánchez e Peña (1997) verificaram que para o modelos ARIMA o procedimento pode não ser eficaz em análises de múltiplos outliers.

Os primeiros procedimentos para deteç̧ão de outliers utilizaram a abordagem clássica. Uma abordagem bayesiana para detectar e estimar valores aberrantes em modelos autoregressivos foi apresentada por McCulloch e Tsay (1994). Neste artigo, utilizando amostrador de Gibbs, eles demonstram a eficácia da metodologia quando os outliers ocorrem isoladamente.

Os procedimentos iterativos para detectar a presença de vários outliers em uma série são mais ineficientes quando estes outliers ocorrem próximos uns dos outros. Neste caso vamos chamá-los de blocos de outliers como tradução do termo patches utilizado por Justel et al. (2001). Blocos de outliers aditivos podem aparecer em um série temporal por vários motivos como comentado anteriormente. Tsay, Peña e Pankratz (1998) mostraram também que um valor aberrante de inovação multivariado pode gerar um bloco de outliers aditivos em uma série temporal univariada.

Justel et al. (2001) desenvolveram um procedimento baseado no artigo de McCulloch e Tsay (1994) que torna o amostrador de Gibbs mais eficiente no tratamento de conjuntos de outliers. No trabalho foi verificado que, para processos autoregressivos, o procedimento é bastante favorável à detecção e estimação de valores aberrantes mesmo quando esses ocorrem conjuntamente. 
Todos os trabalhos mencionados tratam de modelos lineares ARIMA. Após a popularização dos modelos de volatilidade condicional, especialmente os modelos GARCH (Bollerslev, 1986) e de volatilidade estocástica (Taylor, 1986) vários trabalhos têm sido publicados direcionados à detecção de outliers nestes modelos . Como exemplo para modelos GARCH, podemos citar os trabalhos de Hotta e Tsay (1998), van Dijk et al. (1999), Doornik (2000), Zhang (2002) e Zhang e King (2005). Para modelos de volatilidade estocástica podemos citar Motta e Hotta (2003), Chib et al. (2002), Jacquier et al. (2004) e Omori et al. (2005).

O número de trabalhos tratando de modelos GARCH é maior devido a facilidade de se trabalhar com a verossimilhança nestes modelos em comparação com os modelos de volatilidade estocástica. Entretanto, todos eles adotam uma abordagem mais ou menos iterativa para tratar da presença de múltiplos outliers e não os tratam como blocos. Com o objetivo de minorar este problema nas próximas seções o procedimento proposto por Justel et al. (2001) será estendido ao modelo de volatilidade estocástica. 


\subsection{Deteç̧ão e Estimação de Blocos de Outliers}

Nesta seção iremos apresentar inicialmente a versão bayesiana do modelo de volatilidade estocástica com outliers e algumas abordagens existentes na literatura para estimar e detectar os outliers. Depois apresentaremos o procedimento para deteção dos possíveis blocos de outliers e os algoritmos para calcular a distribuição a posteriori para os possíveis blocos de outliers selecionados anteriormente.

\subsubsection{Modelo de Volatilidade Estocástica Bayesiano com Ou- tliers}

Considere o modelo SV dado como na equação (2.1.13), i.e., da forma

$$
\begin{aligned}
& y_{t}=\delta_{t} \beta_{t}+e^{\frac{h_{t}}{2}} \epsilon_{t} \\
& h_{t}=\mu+\phi\left(h_{t-1}-\mu\right)+\sigma_{\eta} \eta_{t},
\end{aligned}
$$

$t=1, \ldots, n$ e denote por $\mathbf{y}=\left(y_{1}, \ldots, y_{n}\right)^{\prime}$ o vetor $n$-dimensional de retornos, $\mathbf{h}=$ $\left(h_{1}, \ldots, h_{n}\right)$, o vetor de volatilidades e $\Psi=\left(\mu, \phi, \sigma_{\eta}^{2}\right)^{\prime}$ o vetor de parâmetros da volatilidade.

A variável aleatória $\delta_{t}$ é uma variável indicadora de posição do valor aberrante, ou seja, $\delta_{t} \sim \operatorname{Ber}(\kappa)$, e adotamos como distribuição a priori $\kappa \sim \operatorname{Beta}\left(u_{0}, n_{0}\right)$. A variável aleatória $\beta_{t}$ é o tamanho do valor espúrio detectado pela variável dicotômica $\delta_{t}$ e sua distribuição a priori será normal com:

$$
\log \left(1+\beta_{t}\right) \sim N\left(-0.5 \nu^{2}, \nu^{2}\right)
$$

$$
\nu \sim L N\left(\nu_{0}, N_{0}\right) .
$$


Os valores dos hiperparâmetros $u_{0}, n_{0}, \nu_{0}$ e $N_{0}$ serão discutidos quando o método for aplicado. As distribuições a priori para complementar a especificação dos modelos serão dadas na seção 3.3 que apresenta os algoritmos para estimação do modelo.

Jacquier et al. (1994) propuseram a análise do modelo de VE com enfoque bayesiano, explorando esta estrutura hierárquica do modelo. A partir deste trabalho, muitas modificações foram propostas. Dentre estes Shephard e Pitt (1997) consideraram amostragem por blocos na tentativa de diminuir os efeitos de dependência nos passos da simulação Monte Carlo Markov Chain (MCMC). Esses pesquisadores também estimaram os parâmetros considerando um conjunto de distribuições a priori diferente do proposto por Jacquier et al. (1994).

Kim et al. (1998) utilizaram mistura de distribuições normais e, condicionada a distribuição da mistura, utilizaram o filtro de Kalman para amostrar todo o vetor de volatilidades de uma única vez. Além desse aperfeiçoamento eles propuseram o uso de amostragem conjunta dos parâmetros para reduzir problemas de dependência.

Chib et al. (2002) generalizaram o método proposto por Kim et al. (1998) de forma a considerar extensões tais como a apresentada na Equação (2.1.13). Eles incluiram covariáveis e modificaram as distribuições das perturbações do modelo de forma a capturar efeitos de valores espúrios em séries financeiras.

Existem algumas diferenças entre os trabalhos mencionados anteriormente. Uma comparação entre algumas destas abordagens é apresentada em Motta e Hotta (2003) e em Liesenfeld e Richard (2003).

Como os procedimentos de deteç̧ão e estimação de conjunto de valores espúrios em modelos de volatilidades, assim como para modelos AR, são baseados na distribuição a posteriori de $\delta_{t}$ e de $\beta_{t}$ precisamos estimá-los. A distribuição a posteriori marginal 
dos elementos de $\delta$ é dada por:

$$
\begin{aligned}
p_{t} & =\mathbb{P}\left(\delta_{t}=1 \mid \mathbf{y}\right)=\sum \mathbb{P}\left(\delta_{r_{t}} \mid \mathbf{y}\right) \quad t=2, \ldots, n \\
& =\sum \int \mathbb{P}\left(\delta_{r_{t}} \mid \mathbf{y}, \Psi\right) \mathbb{P}(\Psi \mid \mathbf{y}) d \Psi \\
& \propto \sum \int \mathbb{P}\left(\mathbf{y} \mid \delta_{r_{t}}, \Psi\right) \mathbb{P}\left(\delta_{r_{t}}\right) \mathbb{P}(\Psi \mid \mathbf{y}) d \Psi,
\end{aligned}
$$

onde a soma é sobre todas as $2^{n-1}$ configurações de outliers. A mesma notação será utilizada em todo o capítulo. A posteriori do tamanho do valor espúrio é dada por:

$$
\mathbb{P}\left(\beta_{t} \mid \mathbf{y}\right)=\sum \mathbb{P}\left(\delta_{r} \mid \mathbf{y}\right) \mathbb{P}\left(\beta_{t} \mid \mathbf{y}, \delta_{r}\right) \quad t=2, \ldots, n
$$

Como temos que determinar uma amostra da distribuição obtida através de uma mistura de densidades, McCulloch e Tsay (1994), no caso de modelos autoregressivos, utilizaram o amostrador de Gibbs para amostrar das posterioris de $\delta_{t}$ e $\beta_{t}$.

Também para o caso de modelos autoregressivos Justel et al. (2001) verificaram que o procedimento de amostrar da distribuição marginal proposto por McCulloch e Tsay (1994) é eficiente no caso em que os valores aberrantes ocorrem isoladamente. Por outro lado, quando os outliers ocorrem em blocos o algoritmo é muito ineficiente e para contornar o problema Justel et al. (2001) propuseram algoritmo composto por duas etapas. Primeiro são identificados os possíveis blocos de outliers e posteriormente realizada a amostragem conjunta dos valores espúrios dentro dos blocos selecionados anteriormente.

Na subseção 3.2.2 será apresentado o procedimento para identificar os possíveis blocos de outliers e posteriormente, na subseção 3.2.3, os resultados necessários para amostragem conjunta dentro dos blocos selecionados anteriormente. 


\subsubsection{Procedimento para Detecção de Blocos}

Para aplicar o método é necessário inicialmente definir os blocos onde serão procurados os outliers, isto é, os locais e duração dos possíveis blocos de outliers. Entretanto, como eles são desconhecidos, primeiro temos que procurar um procedimento para definir os locais e tamanhos dos possíveis blocos. Depois de definidos os possíveis locais dos blocos de outliers podemos aplicar um procedimento para testar se estamos ou não na presença de um bloco de outliers e quais as observações afetadas por outliers. Nesta subseção apresentaremos um procedimento para definir os locais e tamanhos dos possíveis blocos. O procedimento consiste na aplicação de um teste preliminar onde inicialmente são necessários definir dois valores críticos $c_{1}$ e $c_{2} \operatorname{com} c_{2}<c_{1} \mathrm{e}$ $h$ para definir uma largura preliminar dos possíveis blocos. O procedimento consiste dos seguintes passos:

1. Estime o modelo e denote por $\hat{p}_{t}$ a média da distribuição a posteriori de $\delta_{t} \mid \mathbf{y}$ que é a probabilidade de existir um outlier na $t$-ésima observação;

2. A $t_{i}$-observação, $y_{t_{i}}$ é considerada suspeita de ser outlier se $\hat{p}_{t}>c_{1}$. Seja $T^{*}=$ $\left\{t_{1}, \cdots, t_{m}\right\}$ o conjunto detectado inicialmente como possíveis outliers. Se o número de outliers encontrados for maior que $n / 2$ devemos aumentar o valor de $c_{1}$ e voltar ao primeiro passo;

3. Considere agora o segundo ponto crítico $c_{2} \leq c_{1}$ para testar as $2 \mathrm{~h}$ observações em torno de cada uma das $m$ observações encontradas anteriormente. Para todas as observações dentro das janelas teste se $\hat{p}_{t}>c_{2}$;

4. Dentro de cada janela, por exemplo, da janela relacionada a $y_{t_{i}}$, seleciona-se a observação mais distante e anterior a $y_{t_{i}} \operatorname{com} \hat{p}_{t}>c_{2}$ como sendo o ponto inicial 
do conjunto de outliers e denotamos por $y_{t_{i}-k_{i}}$. Analogamente, o ponto mais distante e posterior a $y_{t_{i}} \operatorname{com} \hat{p}_{t}>c_{2}$ é considerado o ponto final do conjunto de valores aberrantes e será denotado por $y_{t_{i}+v_{i}}$. Portanto, o possível bloco de outliers relacionado à observação $y_{t_{i}}$ é dado por $\left(y_{t_{i}-k_{i}}, \cdots, y_{t_{i}+v_{i}}\right)$. Observe que, por enquanto, $\max \left(k_{i}, v_{i}\right) \leq h$. Observe, também, que podemos ter blocos de tamanho 1.

5. Quaisquer conjuntos consecutivos ou sobrepostos são concatenados em um único conjunto de valores espúrios. Desta forma, podemos ao final ter menos do que $m$ blocos e a largura dos blocos é variável;

6. Caso o total de observações dentro dos blocos seja maior que $n / 2$ devemos aumentar o valor crítico $c_{2}$ e reespecificar os possíveis conjuntos de valores. Se isso não for suficiente tomamos um valor para $h$ menor do que especificado inicialmente.

7. Com os provávies blocos identificados é necessário estimar as distribuições a posteriori dentro de cada bloco (com largura maior ou igual a 2). A próxima subseção descreve como isto pode ser realizado utilizando o Amostrador de Gibbs.

\subsubsection{Distribuições a Posteriori para Blocos de Outliers}

Após a identificação dos conjuntos de outliers, é mais razoável retirarmos amostras através do Amostrador de Gibbs dentro dos blocos obtidos. Para tanto, devemos encontrar as densidades a posteriori dos blocos com início no tempo $j$ e de tamanho $k$, i.e., $\delta_{j, k}=\left(\delta_{j}, \ldots, \delta_{j+k-1}\right)^{\prime}$ e $\beta_{j, k}=\left(\beta_{j}, \ldots, \beta_{j+k-1}\right)^{\prime}$ vetores de indicadores e magnitudes dos outliers, respectivamente. 
Inicialmente encontramos as distribuições posterioris marginais completas a fim de identificarmos os conjunto de valores aberrantes. Assim, a partir de nossas informações a priori, o objetivo é analisar a densidade posteriori $\pi\left(h,\left\{\delta_{t}\right\},\left\{\psi_{t}\right\}, \psi, k, \nu \mid y\right)$, que é proporcional ao produto das prioris dos parâmetros $\pi(\psi) \pi(k) \pi(\nu) \mathrm{e}$

$$
\prod_{t=1}^{n} \mathbb{P}\left(y_{t} \mid\left\{h_{t}\right\},\left\{\delta_{t}\right\},\left\{\psi_{t}\right\}, \psi, k, \nu\right) \mathbb{P}\left(h_{t} \mid\left\{\delta_{t}\right\},\left\{\psi_{t}\right\}, \psi, k, \nu\right) \mathbb{P}\left(\delta_{t} \mid\left\{\psi_{t}\right\}, \psi, k, \nu\right) \mathbb{P}\left(\psi_{t} \mid \psi, k, \nu\right)
$$

Como não temos uma forma fechada para essa densidade, será utilizado a técnica de MCMC para amostrar de tal distribuição e o método proposto por Kim et al. (1998). Reescreva o modelo como:

$$
\begin{aligned}
& y_{t}^{*}=h_{t}+z_{t} \\
& h_{t}=\mu+\phi\left(h_{t-1}-\mu\right)+\sigma_{\eta} \eta_{t},
\end{aligned}
$$

onde temos

$$
y_{t}^{*}=\log \left[y_{t}-\left(e^{\psi_{t}}-1\right) \delta_{t}\right]^{2}
$$

e a distribuição de $z_{t}=\log \epsilon_{t}^{2}$ é aproximada por mistura de normais. As marginais completas procuradas são dadas por:

$$
\mathbb{P}\left(\delta_{j}=1 \mid \mathbf{y},\left\{h_{t}\right\},\left\{\psi_{t}\right\}, \psi, k, \delta_{(j)}\right)=\frac{k f_{N}\left(y_{t} \mid \beta_{t}, e^{h_{t}}\right)}{k f_{N}\left(y_{t} \mid \beta_{t}, e^{h_{t}}\right)+(1-k) f_{N}\left(y_{t} \mid 0, e^{h_{t}}\right)}
$$


onde $\delta_{(j)}$ é obtido de $\delta$ eliminando o componente $\delta_{j}$ e $f_{N}\left(y_{t} \mid \mu, \sigma^{2}\right)$ denota que $y_{t}$ tem distribuição normal com média $\mu$ e variância $\sigma^{2}$. Para a magnitude do outlier temos que $\psi_{t} \mid \mathbf{y}, \psi, \delta, \psi_{(j)}, h_{t} \sim N\left(\psi_{j}^{*}, \Psi^{-1}\right)$ onde

$$
\Psi=\frac{\sigma_{\eta}^{2}+\delta_{t}^{2}}{\nu^{2} \sigma_{\eta}^{2}} \quad \text { e } \quad \psi_{t}^{*}=\frac{-0.5 \sigma_{\eta}^{2}+\delta_{t} y_{t}}{\Psi \sigma_{\eta}^{2}} .
$$

Precisamos da distribuição conjunta para amostrarmos o conjunto de outliers e suas respectivas magnitudes. Considere a partição do vetor de parâmetros do modelo como

$$
\theta_{B}=\left(\psi,\left\{h_{t}\right\}, \delta_{2}, \ldots, \delta_{j, k}^{\prime}, \delta_{j+k}, \ldots, \delta_{n}, \beta_{2}, \ldots, \beta_{j, k}^{\prime}, \beta_{j+k}, \ldots, \beta_{n},\right)^{\prime}
$$

Portanto, precisamos da distribuição condicional posteriori de $\delta_{j, k}$ e $\beta_{j, k}$, onde temos que

$$
\mathbb{P}\left(\delta_{j, k} \mid \mathbf{y}, \theta_{\delta_{j, k}}\right) \propto f\left(\mathbf{y} \mid \theta_{\delta_{j, k}} ; \delta_{j, k}\right) \cdot \kappa^{s_{j, k}}(1-\kappa)^{k-s_{j, k}}
$$

onde $s_{j, k}=\sum_{t=j}^{j+k-1} \delta_{t}$. A verossimilhança pode ser fatorada como:

$$
f\left(\mathbf{y} \mid \theta_{\delta_{j, k}} ; \delta_{j, k}\right)=f\left(\mathbf{y}_{2}^{j-1} \mid \theta_{\delta_{j, k}}\right) \cdot f\left(\mathbf{y}_{j}^{T_{j, k}} \mid \mathbf{y}_{2}^{j-1} ; \theta_{\delta_{j, k}} ; \delta_{j, k}\right) \cdot f\left(\mathbf{y}_{T_{j, k+1}}^{n} \mid \mathbf{y}_{2}^{T_{j, k}} ; \theta_{\delta_{j, k}}\right),
$$

onde $\mathbf{y}_{j}^{k}=\left(y_{j}, \ldots, y_{k}\right)^{\prime}$ e $T_{j, k}=\min \{n, j+k\}$.

Para o modelo da Equação (2.1.13) temos que a densidade será

$$
f\left(\mathbf{y}_{j}^{T_{j, k}} \mid \mathbf{y}_{2}^{j-1} ; \theta_{\delta_{j, k}} ; \delta_{j, k}\right) \propto \prod_{t=j}^{T_{j, k}} f_{N}\left(y_{t}\left|h_{t}, \psi\right| \delta_{t} \psi_{t} ; e^{h_{t}}\right) f_{N}\left(h_{t}\left|h_{(t)}, \psi\right| \mu+\phi\left(h_{t-1}-\mu\right) ; \sigma_{\eta}\right)
$$

Para o modelo da Equação (2.1.14) a densidade será proporcional a

$$
f\left(\mathbf{y}_{j}^{T_{j, k}} \mid \mathbf{y}_{2}^{j-1} ; \theta_{\delta_{j, k}} ; \delta_{j, k}\right) \propto \prod_{t=j}^{T_{j, k}} f_{N}\left(y_{t}\left|h_{t}, \psi\right| 0 ; e^{h_{t}}\right) f_{N}\left(h_{t}\left|h_{(t)}, \psi\right| \delta_{t} \psi_{t}+\mu+\phi\left(h_{t-1}-\mu\right) ; \sigma_{\eta}\right) .
$$


Obtida a verossimilhança anteriormente, encontra-se a posteriori condicional de $\beta_{j, k}$ que é dada por

$$
\mathbb{P}\left(\beta_{j, k} \mid \mathbf{y}, \theta_{\delta_{j, k}}\right) \propto f\left(\mathbf{y} \mid \theta_{\beta_{j, k}} ; \beta_{j, k}\right) \cdot \mathbb{P}\left(\beta_{j, k}\right)
$$

O segundo amostrador de Gibbs é implementado a partir desses resultados. Após detectarmos os conjuntos de valores espúrios, utilizamos as posterioris condicionais encontradas para obtermos amostras de $\delta_{j, k}$ e $\beta_{j, k}$.

\subsection{Estimação dos Parâmetros do Modelo}

Para a análise Bayesiana do modelo são formuladas as distribuições a priori para os parâmetros. Jacquier et al. (1994), Shephard e Pitt (1997) e Kim et al. (1998), visando facilitar os cálculos e aumentar a eficiência dos resultados, propuseram prioris conjugadas para todos os coeficientes do modelo.

Neste trabalho serão consideradas as estimativas dos parâmetros obtidas através do algoritmo proposto em Kim et al. (1998), onde é considerado a amostragem conjunta das volatilidades e amostragem em pares dos parâmetros de forma a contornar alguns problemas na eficiência computacional.

\subsubsection{Prioris para os Parâmetros do Modelo}

Considere o vetor $\psi=\left(\mu, \phi, \sigma_{\eta}^{2}\right)$ denotando os parâmetros do modelo e assumindo que os parâmetros possuem distribuições a priori independentes. Inicialmente o parâmetro $\phi$ é amostrado da densidade condicional completa através do método da Rejeição. Assim, considerando a reparametrização, $\phi=2 \phi^{*}-1$, onde a distribuição de $\phi$ é uma beta com parâmetros $\left(\phi^{(1)}, \phi^{(2)}\right)$ e a priori de $\phi$ é dada por

$$
\pi(\phi) \propto\left\{\frac{(1+\phi)}{2}\right\}^{\phi^{(1)}-1}\left\{\frac{(1-\phi)}{2}\right\}^{\phi^{(2)}-1}, \quad \phi^{(1)}, \phi^{(2)}>\frac{1}{2},
$$


A distribuição a priori para $\mu$ é dada ser Normal com parâmetros $\left(\mu_{0}, M_{0}\right)$.

\subsubsection{Distribuições Posterioris dos Parâmetros}

Amostrando $\sigma_{\eta}^{2}, \phi$. A amostragem de $\sigma_{\eta}^{2}$ e $\phi$ feita por Kim et al. (1998) é direta. Eles assumem, como Shephard e Pitt (1997), uma priori conjugada $\sigma_{\eta}^{2} \mid \phi, \mu \sim$ $\mathcal{I} G\left(\sigma_{r} / 2, S_{\sigma} / 2\right)$, Assim, amostra-se de uma Gama-inversa:

$$
\sigma_{\eta}^{2} \mid \mathbf{y}, \mathbf{h}, \phi, \mu \sim \mathcal{I} G\left\{\frac{n+\sigma_{r}}{2}, \frac{S_{\sigma}+\left(h_{1}-\mu\right)^{2}\left(1-\phi^{2}\right)+\sum_{t=1}^{n-1}\left(\left(h_{t+1}-\mu\right)-\phi\left(h_{t}-\mu\right)\right)^{2}}{2}\right\},
$$

fixando-se $\sigma_{r}=5$ e $S_{\sigma}=0.01 \times \sigma_{r}$.

Dada a priori (2.1.12), a densidade condicional completa de $\phi$ é proporcional a

$$
\pi(\phi) f\left(h \mid \mu, \phi, \sigma_{\eta}^{2}\right)
$$

onde

$$
\log f\left(h \mid \mu, \phi, \sigma_{\eta}^{2}\right) \propto-\frac{\left(h_{t}-\mu\right)^{2}(1-\phi)}{2 \sigma_{\eta}^{2}}+\frac{1}{2} \log \left(1-\phi^{2}\right)-\frac{\sum_{t=1}^{n-1}\left\{\left(h_{t+1}-\mu\right)-\phi\left(h_{t}-\mu\right)\right\}^{2}}{2 \sigma_{\eta}^{2}} .
$$

Esta função é côncava em $\phi$ para quaisquer valores de $\phi^{(1)}$ e $\phi^{(2)}$. Portanto, pode-se amostrar $\phi$ usando algoritmo de rejeição. Kim et al. (1998) tomaram a expansão por série de Taylor da priori em torno de

$$
\hat{\phi}=\frac{\sum_{t=1}^{n-1}\left(h_{t+1}-\mu\right)\left(h_{t}-\mu\right)}{\sum_{t=1}^{n-1}\left(h_{t}-\mu\right)^{2}} .
$$

Logo, dado um valor proposto $\phi^{*}$ de $N\left(\hat{\phi}, V_{\phi}\right)$ onde $V_{\phi}=\sigma_{\eta}^{2}\left\{\sum_{t=1}^{n-1}\left(h_{t}-\mu\right)^{2}\right\}^{-1}$ este valor é aceito com probabilidade $\exp \left\{g\left(\phi^{*}\right)-g\left(\phi^{(i-1)}\right)\right\}$, onde

$$
g(\phi)=\log \pi(\phi)-\frac{\left(h_{t}-\mu\right)^{2}\left(1-\phi^{2}\right)}{2 \sigma_{\eta}^{2}}+\frac{1}{2} \log \left(1-\phi^{2}\right) .
$$

Se o valor proposto é rejeitado, tomamos $\phi^{(i)}=\phi^{(i-1)}$. 
Amostrando $\mu$. Supondo uma priori difusa para $\mu$, ele é amostrado de uma distribuição condicional completa dada por

$$
\mu \mid \mathbf{h}, \phi, \sigma_{\eta}^{2} \sim N\left(\hat{\mu}, \sigma_{\mu}^{2}\right),
$$

onde

$$
\hat{\mu}=\sigma_{\mu}^{2}\left\{\frac{\left(1-\phi^{2}\right)}{\sigma_{\eta}^{2}} h_{1}+\frac{(1-\phi)}{\sigma_{\eta}^{2}} \sum_{t=1}^{n-1}\left(h_{t+1}-\phi h_{t}\right)\right\}
$$

e

$$
\sigma_{\mu}^{2}=\sigma_{\eta}^{2}\left\{(n-1)\left(1-\phi^{2}\right)+\left(1-\phi^{2}\right)\right\}^{-1} .
$$




\subsection{Aplicações}

Nesta seção apresentaremos a aplicação do método à séries simuladas e reais. Os resultados obtidos para as séries simuladas $\Psi_{1}$ e $\Psi_{2}$ (sem outlier) e para $\Psi_{3}$ e $\Psi_{4}$ (com outliers) apresentaram comportamentos análogos e para simplificação serão apresentados apenas os resultados relativos ao conjunto de parâmetro $\Psi_{1}$ e $\Psi_{3}$. O conjunto de dados reais utilizados são as séries de retornos do Ibovespa e NYSE apresentadas no Capítulo 2.

\subsubsection{Séries Simuladas}

Para verificar o desempenho do procedimento proposto, utilizamos dados simulados com valores aberrantes e sem a presença desses. Foram consideradas as séries de retornos simuladas com os conjunto de parâmetros dados no Capítulo 2 e o procedimento mostrou-se estável em relação aos diferentes valores de parâmetros utilizados.

O conjunto $\Psi_{1}$ gera uma série com alta persistência e sem a presença de outliers no processo gerador de dados. Por outro lado, o conjunto de parâmetros $\Psi_{3}$ reflete além de alta persistência, a presença de outliers do tipo AO isolados e em blocos, assim como a presença de outliers do tipo IO.

Para iniciar o procedimento devemos definir os hiperparâmetros das distribuições a priori. Na fase inicial, é utilizado o amostrador de Gibbs padrão, ou seja, a amostragem é feita marginalmente e não em conjunto. Nessa primeira etapa consideramos as distribuições a priori, dadas como segue: $\mu \sim N(0,100), \phi^{*} \sim \operatorname{Beta}(20,15)$, $\kappa \sim \operatorname{Beta}(2,100), \sigma_{\eta} \sim I G(2.5,0.05 / 2)$. Para $\delta$ teremos que $\log (\delta) \sim N(-3,0.15)$.

A priori normal para o parâmetro $\log (\delta)$ implica distribuição a priori Log-normal para os parâmetros com média 0.05 e desvio igual a 0.02 . Os hiperparâmetros da 
priori de $\kappa$ implica uma média de 0.0196 e desvio padrão de 0.0136 .

Para evitar problemas de convergência foi tomado um total de 150000 iterações para o primeiro passo do procedimento, onde obtemos estimativas baseados no Amostrador de Gibbs padrão, e o mesmo número de iterações para o Amostrador de Gibbs adaptado.

As estimativas dos parâmetros foram obtidas pela média amostral das últimas 1000 iterações. O uso do Amostrador de Gibbs padrão no primeiro passo do procedimento nos fornece estimativas para os parâmetros das distribuições utilizadas no Amostrador de Gibbs adaptado.

Para a localização de um bloco de outlier, foi tomado $c_{1}=0.5$ para o primeiro passo do algoritmo e $c_{2}=0.3$ para definirmos os limites extremos dos blocos. Para verificarmos quantas observações seriam consideradas num bloco utilizamos largura da janela igual a 5 . O critério foi determinado baseado em uma estrutura autoregressiva, como em nosso caso temos uma estrutura $\operatorname{ARMA}(1,1)$, foi verificado que coerentes modificações no tamanho dessa janela não causam distorções dos resultados.

\subsubsection{Série Sem Outlier e Mudança Estrutural}

As distribuições a posteriori para o modelo simulado com o conjunto de parâmetros $\Psi_{1}$ estão ilustradas na Figura 3.1. A figura apresenta as amostras, histogramas e correlograma para as distribuições dos parâmetros num total de 20000 iterações. A primeira coluna da figura apresenta informações sobre a distribuição a posteriori do parâmetro de persistência $\phi \mid y$ a segunda coluna apresenta as características distribucionais de $\sigma_{\eta} \mid y$ e finalmente a terceira coluna apresenta a posteriori de $\beta \mid y$. Vale lembrar que na série simulada com o conjunto de parâmetro $\Psi_{1}$ não são inseridos valores atípicos no processo gerador de dados. 


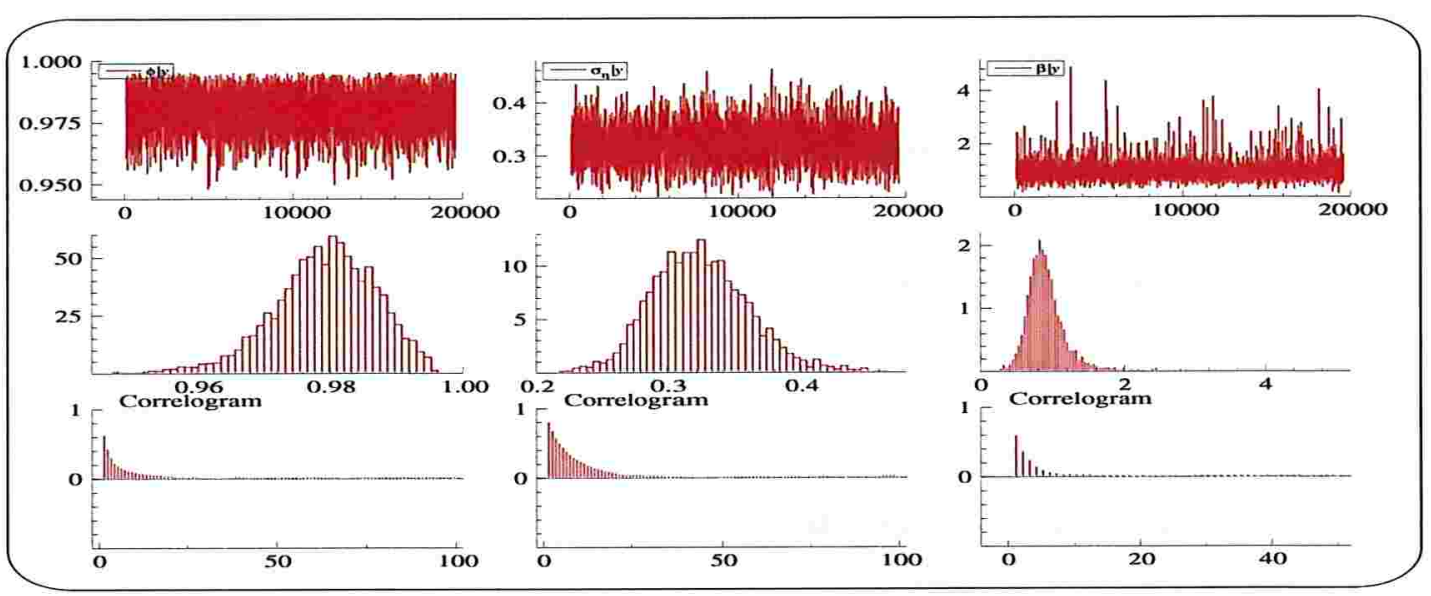

Figura 3.1: Gráficos dos valores amostrados, histogramas das densidades posterioris dos parâmetros e correlograma para amostragem da série simulada sem outliers, $\Psi_{1}$.

A Figura 3.2 apresenta a série de retornos simulada a partir do conjunto de parâmetros $\Psi_{1}$ na parte superior. Na parte inferior é apresentado o resultado do Passo 1, i.e., a média da distribuição posteriori marginal, $\left(\delta_{t} \mid \mathbf{y}\right)$, para a probabilidade de uma determinada observação ser um outlier. Por questão de simplicidade, embora com abuso de linguagem, muitas vezes chamaremos esta média apenas de estimativa da probabilidade a posteriori na presença de outlier. Pode-se notar que para a série simulada sem outlier o primeiro passo do algoritmo foi suficiente para verificarmos a inexistência de valores atípicos. Através da Figura 3.2 verificamos que todos os valores da probabilidade de uma observação ser um outlier foram menores que $c_{1}$, tomando como sendo igual a 0.5 . 


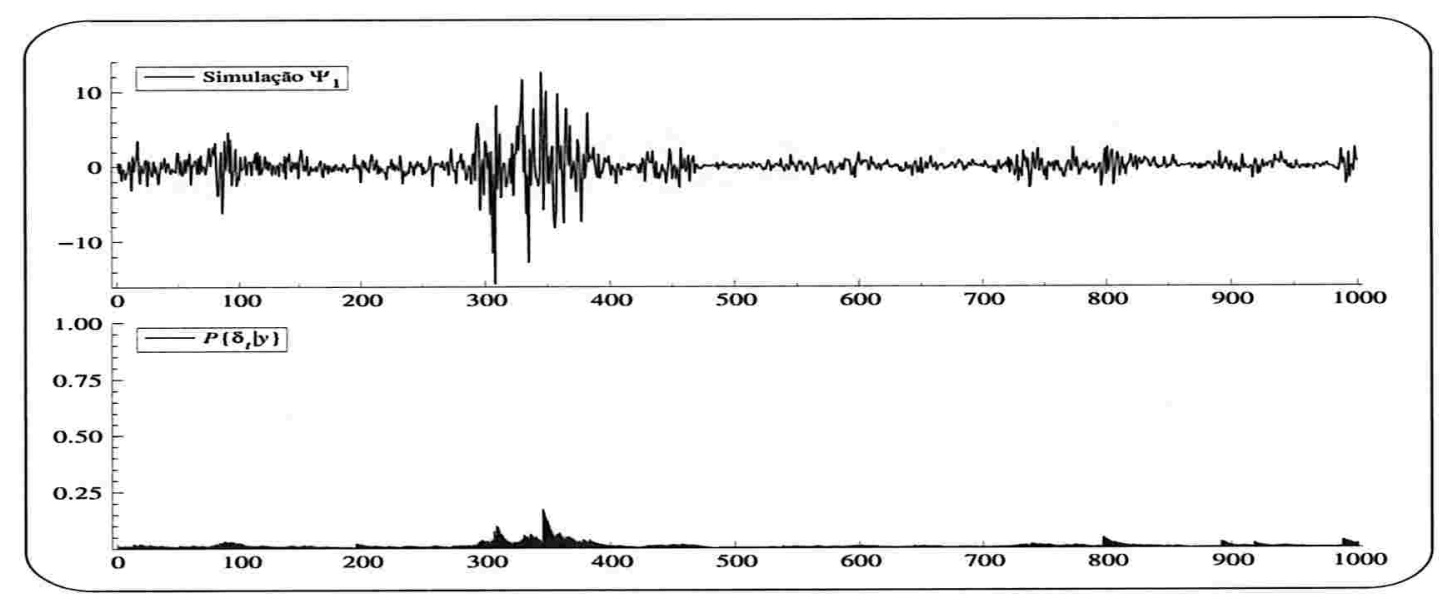

Figura 3.2: a) Retornos. b) Média da distribuição posteriori marginal do passo 1 para deteç̧ão de outliers da série simulada $\Psi_{1}$.

\subsubsection{Série Com Outliers e Mudanças Estruturais}

Agora, devemos verificar o comportamento do método quando tratamos séries com valores espúrios. Nesse caso foram simuladas séries de tamanhos 1000 e conjuntos de parâmetros $\Psi_{3}$.

No conjunto $\Psi_{3}$ temos dois outliers do tipo AO, o primeiro na posição $t=50$ e o segundo em $t=500$, além de um bloco com 6 outliers consecutivos do tipo AO em $t=250$ e um IO na posição $t=700$.

Na Figura 3.3 podemos verificar o comportamento da distribuição a posteriori dos parâmetros obtidas ao estimarmos o modelo quando o processo gerador de dados foi baseado no conjunto de parâmetros $\Psi_{3}$. 


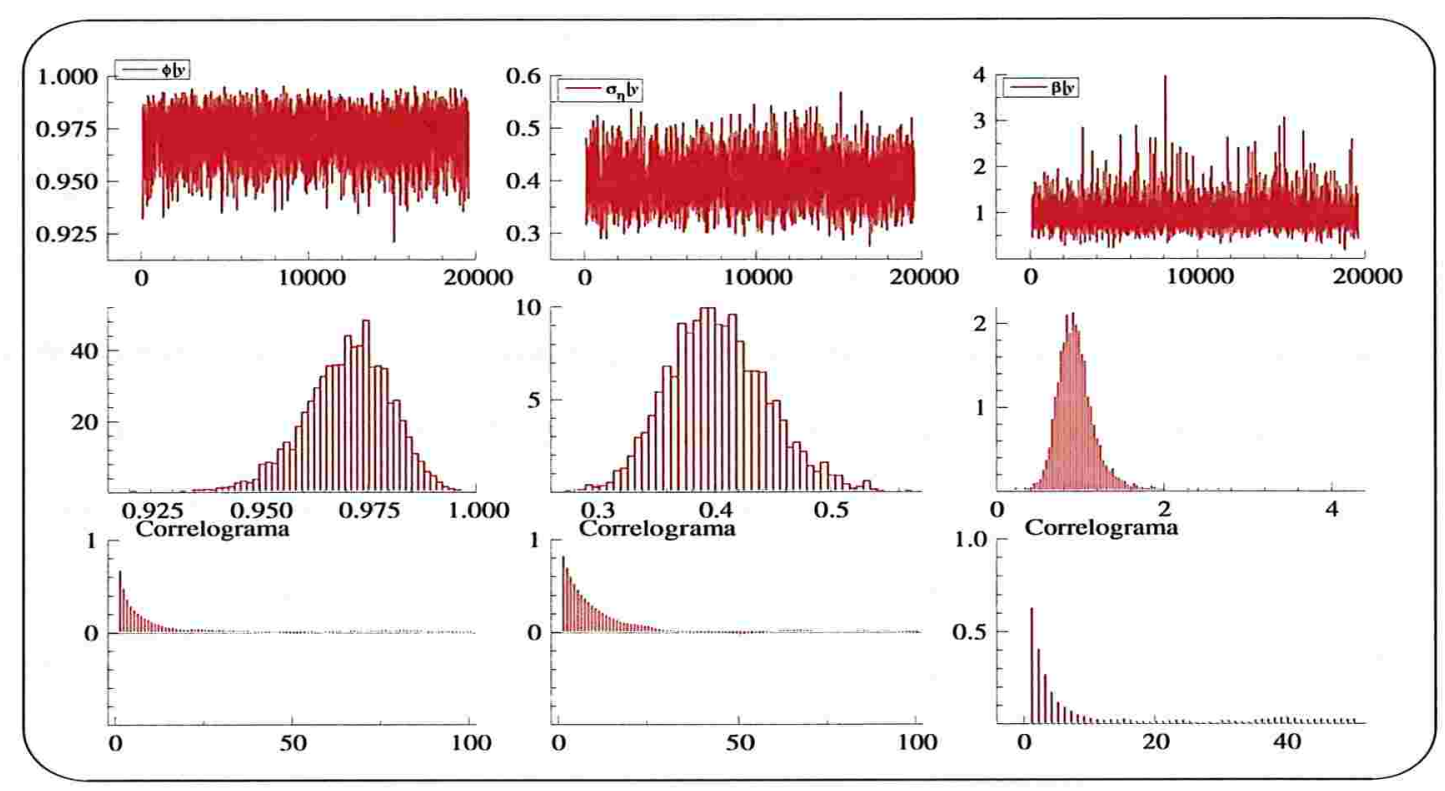

Figura 3.3: Gráficos dos valores amostrados, histogramas das densidades posterioris dos parâmetros e correlograma para amostragem dos parâmetros da série simulada, com outliers, $\Psi_{3}$.

As Figuras 3.4 e 3.5 apresentam a série simulada com o conjunto de parâmetros $\Psi_{3}$, ou seja, com todas as configurações de outliers que consideramos neste trabalho. Como pode ser verificado na parte superior da figura, a série simulada apresenta 2 outliers do tipo AO isolados, um bloco de outliers consecutivos de tamanho 5 e um outlier de inovação, em $t=700$, refletindo a presença destas estruturas no processo gerador de dados. 
No Passo 1 foram detectados apenas dois outliers isolados (blocos de tamanho um) na posição 50 e 500, um bloco de tamanho 6 (entre 250 e 255) e outro de tamanho 6 (entre 700 e 705). No primeiro bloco, que coincide com o bloco de AO's introduzidos nem todas as probabilidades estimadas foram altas. No segundo bloco, que corresponde a presença do IO ocorreu o mesmo. No Passo 7 são reestimadas as probabilidades de existência de outlier dentro dos 2 blocos detectados. Os resultados dos Passos 1 e 7 são apresentados nas Figuras 3.4 e 3.5. Os valores das probabilidades dentro dos blocos são dadas pelo Passo 7 enquanto as de fora dos blocos são as do Passo 1. Nas estimativas do Passo 7 todas as probabilidades dentro do bloco foram estimadas com valores altos.

O gráfico foi separado em duas séries de tamanho 500 para melhor visualização dos blocos de valores espúrios.

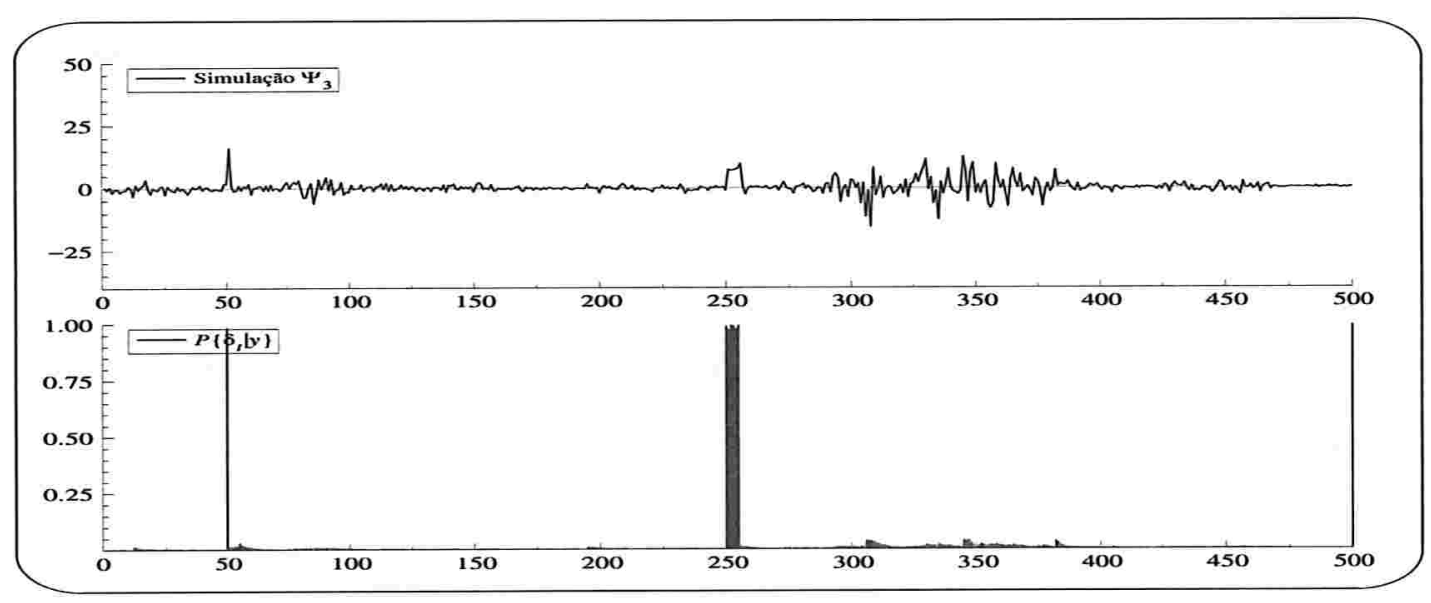

Figura 3.4: Deteç̧ão de blocos de outliers da série simulada $\Psi_{3} 500$ primeiras observações. a) Retornos. b) Média da distribuição posteriori marginal do passo 7 . 


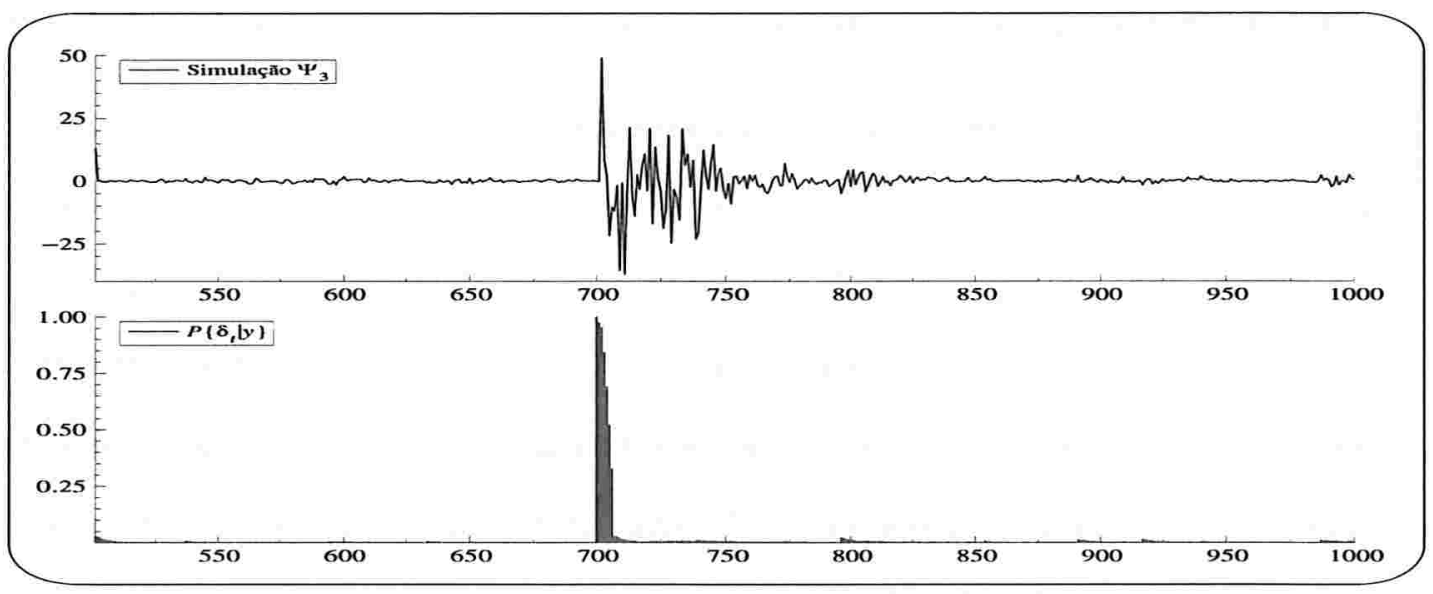

Figura 3.5: Detecção de blocos de outliers da série simulada $\Psi_{3} 500$ observações finais. a) Retornos. b) Média da distribuição posteriori marginal do passo 7 .

Podemos verificar que todos os outliers inseridos foram detectados pelo procedimento com alta probabilidade a posteriori. Os valores espúrios que foram considerados ocorrendo isoladamente são detectados perfeitamente, assim como o bloco que foi inserido no tempo $t=250$. Apesar de considerarmos o procedimento eficaz quando tratamos os outliers do tipo $\mathrm{AO}$, verificamos que o método destaca a ocorrência de um valor atípico no tempo $t=700$, onde foi inserido um IO. Entretanto, este foi detectado como um bloco de AO's com tamanhos decrescentes e média das probablidades posteriori de ser um AO também decrescente. 


\subsubsection{Estimativas dos Parâmetros, Posição e Tamanho dos Outliers}

Nesta subseção serão apresentadas as estimativas dos parâmetros, das localizações dos outliers e seus respectivos tamanhos. São apresentadas as médias e os desvios padrões das distribuições a posteriori de cada parâmetro. Os resultados são apresentados na Tabela 3.1, vale lembrar que estaremos nos referindo as médias e desvios das distribuições a posteriori apenas como estimativas. Excetos pelos valores detectados após o IO, nenhuma observação foi classificada falsamente como outlier, sendo que todas as outras probabilidades estimadas foram menor do que 0.05 .

\begin{tabular}{c|cc}
\hline \hline \multirow{2}{*}{ Modelos } & GS & GS \\
& Padrão & Adaptado \\
\hline \multirow{4}{*}{$\Psi_{1}$} & $\phi=0.98176$ & 0.98046 \\
& $(0.0082045)$ & $(0.00807)$ \\
& $\sigma_{\eta}=0.1108$ & 0.1143 \\
& $\beta \underset{(0.02945)}{=0.96142}$ & $(0.02217)$ \\
& 0.98567 \\
& $(0.08729)$ & $(0.11421)$ \\
& & \\
& $\phi=0.94601$ & 0.95259 \\
$\Psi_{3}$ & $(0.00635)$ & $(0.0135)$ \\
& $\sigma_{\eta}=0.21652$ & 0.25358 \\
& $\beta=1.03453)$ & $(0.034207)$ \\
& $\beta=1.05172$ & 1.03173 \\
$(0.06824)$ & $(0.11071)$ \\
\hline \hline
\end{tabular}

Tabela 3.1: Média das distribuições a posteriori dos parâmetros para $\Psi_{1}$ e $\Psi_{3}$ e desvios padrões entre parênteses. 
A Tabela 3.2 apresenta os valores estimados para os tamanhos dos outliers identificados. Eles indicam todos os outliers inseridos no processo gerador de dados. Pode-se notar que o Amostrador de Gibbs padrão (Passo 1), onde amostramos da posteriori marginal, consegue capturar os valores atípicos que ocorrem isoladamente. Por outro lado não é eficaz quando esses valores ocorrem em blocos. Ao analisarmos as estimativas dos valores obtidos pelo Amostrador de Gibbs adaptado (Passo 7), onde amostramos da posteriori conjunta, podemos ver que o método demonstra ser satisfatório na caracterização de valores atípicos isolados e em blocos.

\begin{tabular}{|c|c|c|c|c|}
\hline Outliers & $\begin{array}{c}\text { GS } \\
\text { Padrão }\end{array}$ & $\begin{array}{c}\text { GS } \\
\text { Adaptado }\end{array}$ & $\begin{array}{l}\text { Tamanho } \\
\text { Real }\end{array}$ & $\begin{array}{c}\text { Média da Distribuição } \\
\text { Posteriori }\end{array}$ \\
\hline$\Delta_{50}$ & $\begin{array}{l}8.0614 \\
(0.9431)\end{array}$ & 8.0322 & 8 & 1.00 \\
\hline$\Delta_{250}$ & 4.6131 & 4.0352 & 4 & 1.00 \\
\hline$\Delta_{251}$ & $\begin{aligned} 2.6140 \\
(0.3421)\end{aligned}$ & $\begin{array}{r}3.9816 \\
(0.2667)\end{array}$ & 4 & 0.98 \\
\hline$\Delta_{252}$ & 2.8376 & $\begin{array}{l}4.0213 \\
(0.3134)\end{array}$ & 4 & 1.00 \\
\hline$\Delta_{253}$ & $\begin{array}{r}4.9647 \\
(0.4301)\end{array}$ & $\begin{array}{r}4.0199 \\
(0.3353)\end{array}$ & 4 & 1.00 \\
\hline$\Delta_{254}$ & $\begin{array}{l}3.2369 \\
(0.7012)\end{array}$ & $\begin{array}{r}4.0325 \\
(0.6831)\end{array}$ & 4 & 0.99 \\
\hline$\Delta_{255}$ & 3.7932 & 4.3621 & 4 & 1.00 \\
\hline$\Delta_{500}$ & 5.1321 & 5.0862 & 5 & 1.00 \\
\hline$\Lambda_{700}$ & 8.2311 & 3.9631 & $\mathrm{IO}$ & 1.00 \\
\hline$\Lambda_{701}$ & 7.4581 & $\begin{array}{r}3.8345 \\
(0.1622)\end{array}$ & $\mathrm{IO}$ & 0.99 \\
\hline$\Lambda_{702}$ & 7.2683 & 3.6228 & IO & 0.97 \\
\hline$\Lambda_{703}$ & 5.7162 & 2.6883 & $\mathrm{IO}$ & 0.87 \\
\hline$\Lambda_{704}$ & 5.5363 & 2.6711 & $\mathrm{IO}$ & 0.63 \\
\hline$\Lambda_{705}$ & $\begin{array}{l}4.021 \\
(0.2251)\end{array}$ & $\begin{array}{l}2.125 \\
(0.1498)\end{array}$ & IO & 0.52 \\
\hline
\end{tabular}

Tabela 3.2: Médias das distribuições a posteriori dos tamanhos dos outliers e desvios, tamanho real dos outliers inseridos e média da distribuição a posteriori da probabilidade de uma observação ser outlier. São apresentados os resultados para as observações detectadas com outliers identificados (Prob. a posteriori ¿ 0.5.) 
As Figuras 3.6 e 3.7 apresentam os tamanhos dos outliers detectados. Apesar de estarmos considerando apenas as 1000 últimas iterações para as estimativas, as figuras apresentam o total de 50000 iterações, a fim de obtermos a distribuição a posteriori do tamanho do outlier.

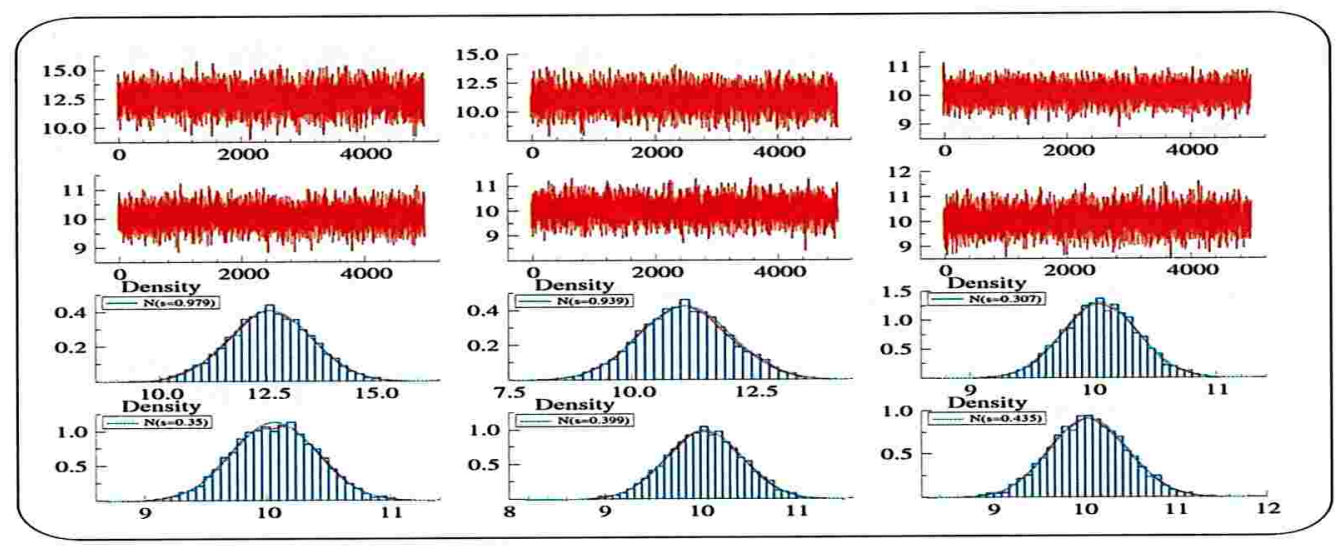

Figura 3.6: Gráfico do tamanho dos outliers detectados na série simulada $\Psi_{2}$ : a,b) Amostra da distribuição a posteriori. c,d) Histograma das distribuições a posteriori dos tamanhos dos outliers.

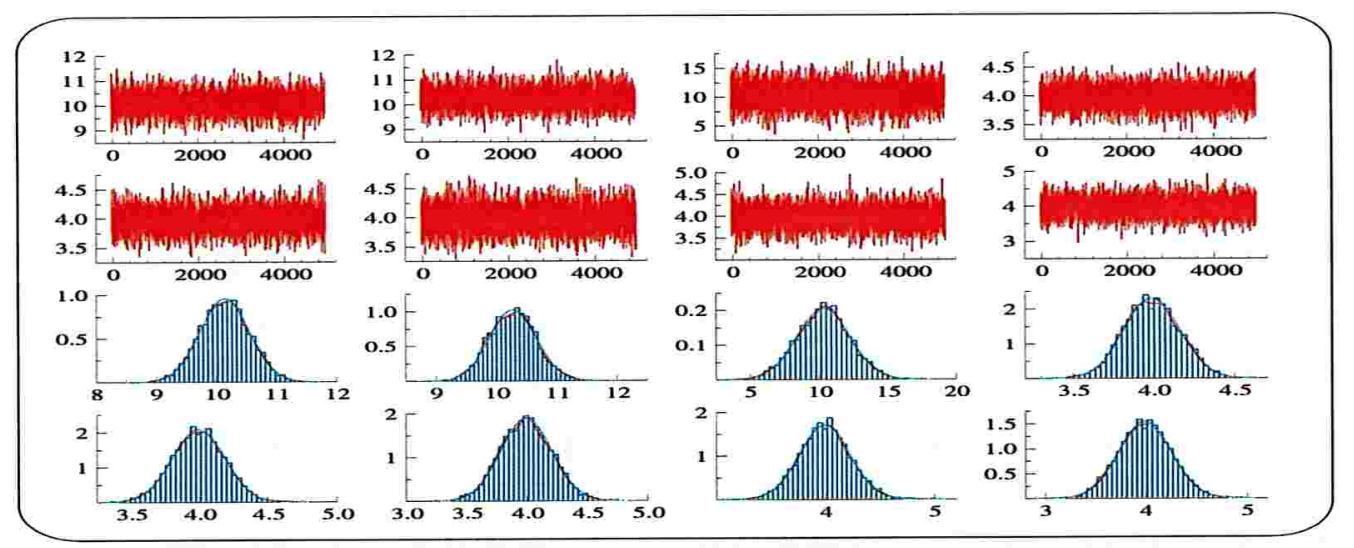

Figura 3.7: Gráfico do tamanho dos outliers detectados na série simulada $\Psi_{2}$ : a,b) Amostra da distribuição a posteriori. c,d) Histograma das distribuições a posteriori dos tamanhos dos outliers. 


\subsubsection{Análise da Série do Ibovespa}

Para a série de retornos do índice Bovespa, completamos o modelo especificando as distribuições a priori. Para $\phi$ foram considerados os valores de $\phi^{(1)}=20$ e $\phi^{(2)}=1.5$. Isso reflete um alto grau de persistência na volatilidade comum em séries financeiras.

A Figura 3.8 nos fornece as estimativas dos parâmetros para o modelo. Apresentamos as últimas 20000 iterações para visualizarmos a distribuição a posteriori dos parâmetros.

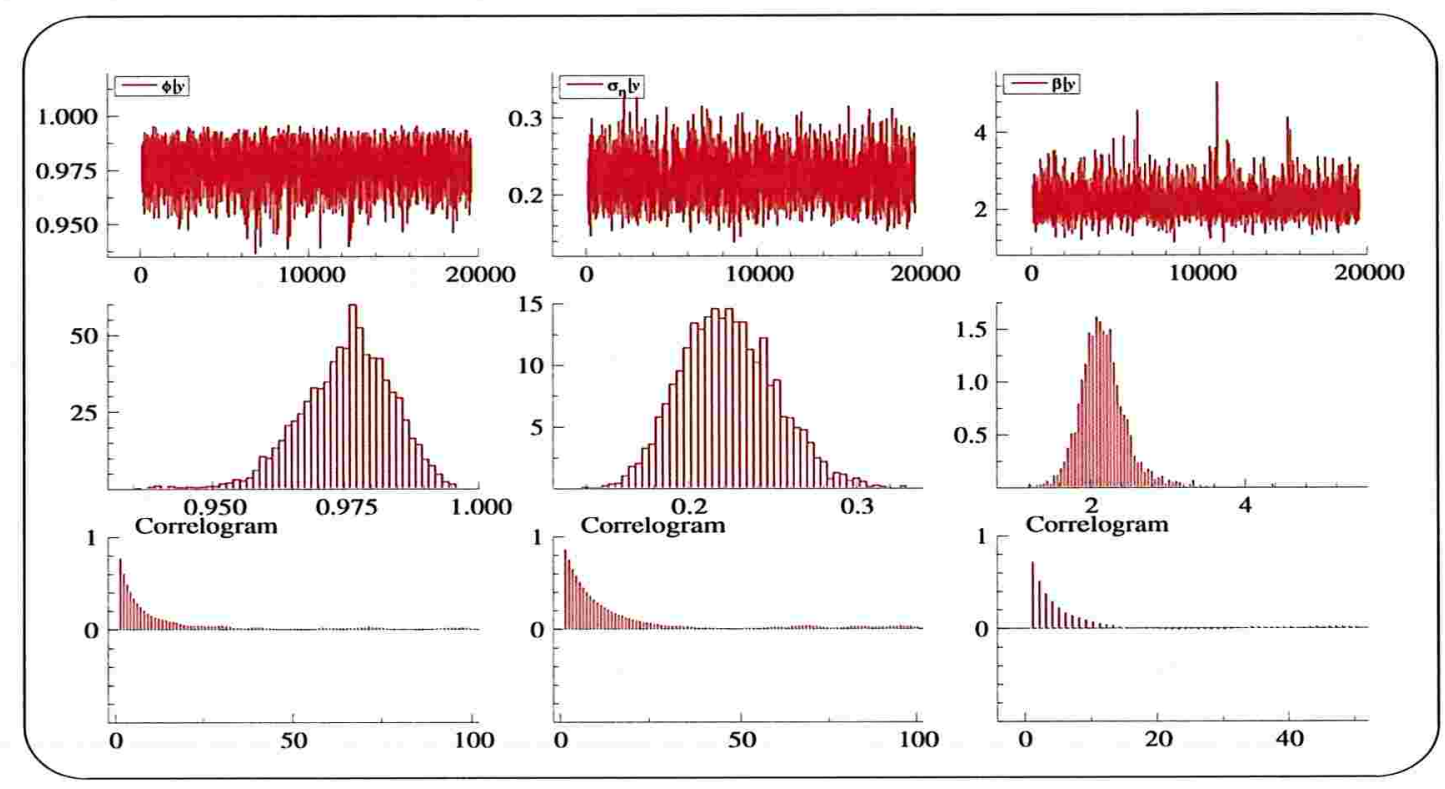

Figura 3.8: Gráficos dos valores amostrados, histogramas das densidades posterioris dos parâmetros e correlograma para amostragem da série simulada da série Ibovespa. 
A Tabela 3.3 apresenta as estimativas dos parâmetros e seus respectivos desvios padrões. Nota-se que as estimativas dos parâmetros são relativamente próximas quando consideramos o amostrador de Gibbs padrão e o adaptado.

\begin{tabular}{c|cc}
\hline \hline \multirow{2}{*}{ Parâmetros } & $\begin{array}{c}\text { GS } \\
\text { Padrão }\end{array}$ & $\begin{array}{c}\text { GS } \\
\text { Adaptado }\end{array}$ \\
\hline$\phi$ & $\underset{0.9719}{(\mathbf{0 . 0 0 7 6 )}}$ & 0.9786 \\
$(\mathbf{0 . 0 0 8 8 )}$ \\
$\sigma_{\eta}$ & 0.0518 & 0.0483 \\
$\beta$ & $\underset{(0.0264)}{(\mathbf{0 . 0 2 5 2 )}}$ \\
$\beta$ & 2.1214 & 2.1690 \\
$(0.2358)$ & $(0.2518)$ \\
\hline \hline
\end{tabular}

Tabela 3.3: Média e desvios das distribuições a posteriori dos parâmetros do modelo para Ibovespa.

Analogamente ao caso anterior, as Figuras 3.9, 3.10 e 3.11 apresentam as estimativas da probabilidade posteriori de um determinado valor ser um outlier. A distribuição a posteriori ilustrada é obtida através do Amostrador de Gibbs Adaptado, ou seja, a partir da amostragem conjunta dos blocos de outliers.

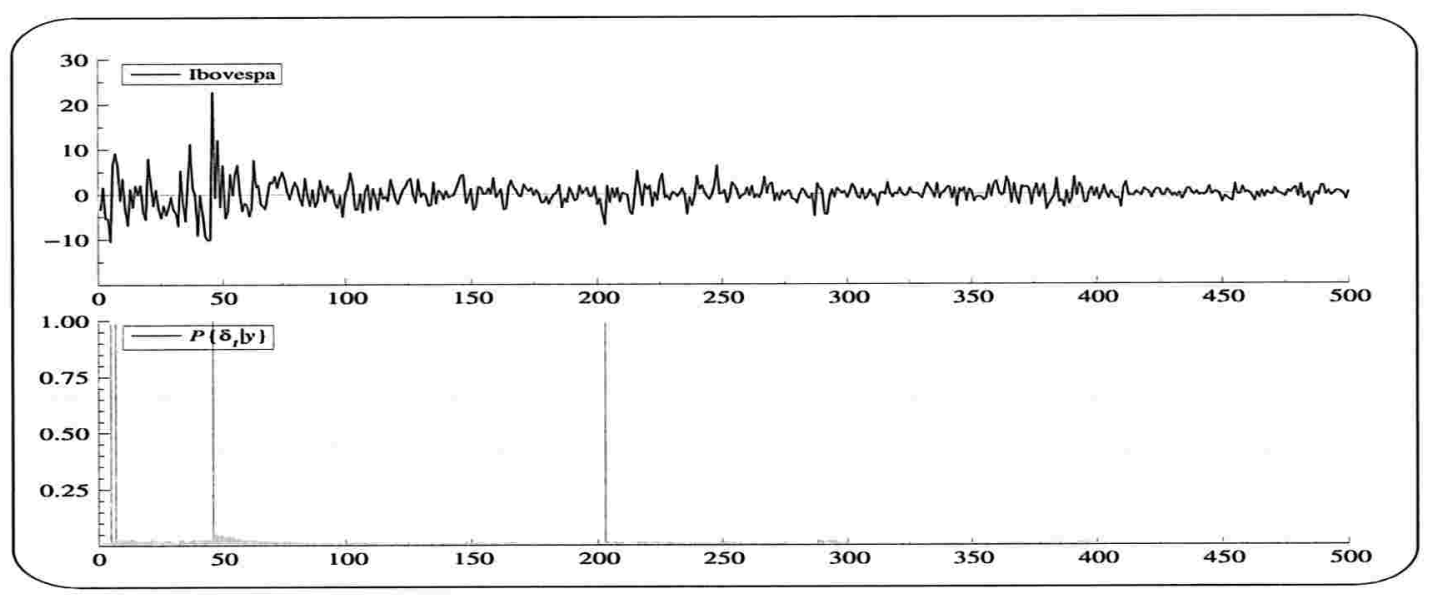

Figura 3.9: Outliers detectados na série Ibovespa: a) Retornos. b) Média da distribuição a posteriori do passo 7 (dentro dos blocos) e ppasso 1 (fora dos blocos) para deteç̧ão de outliers. 


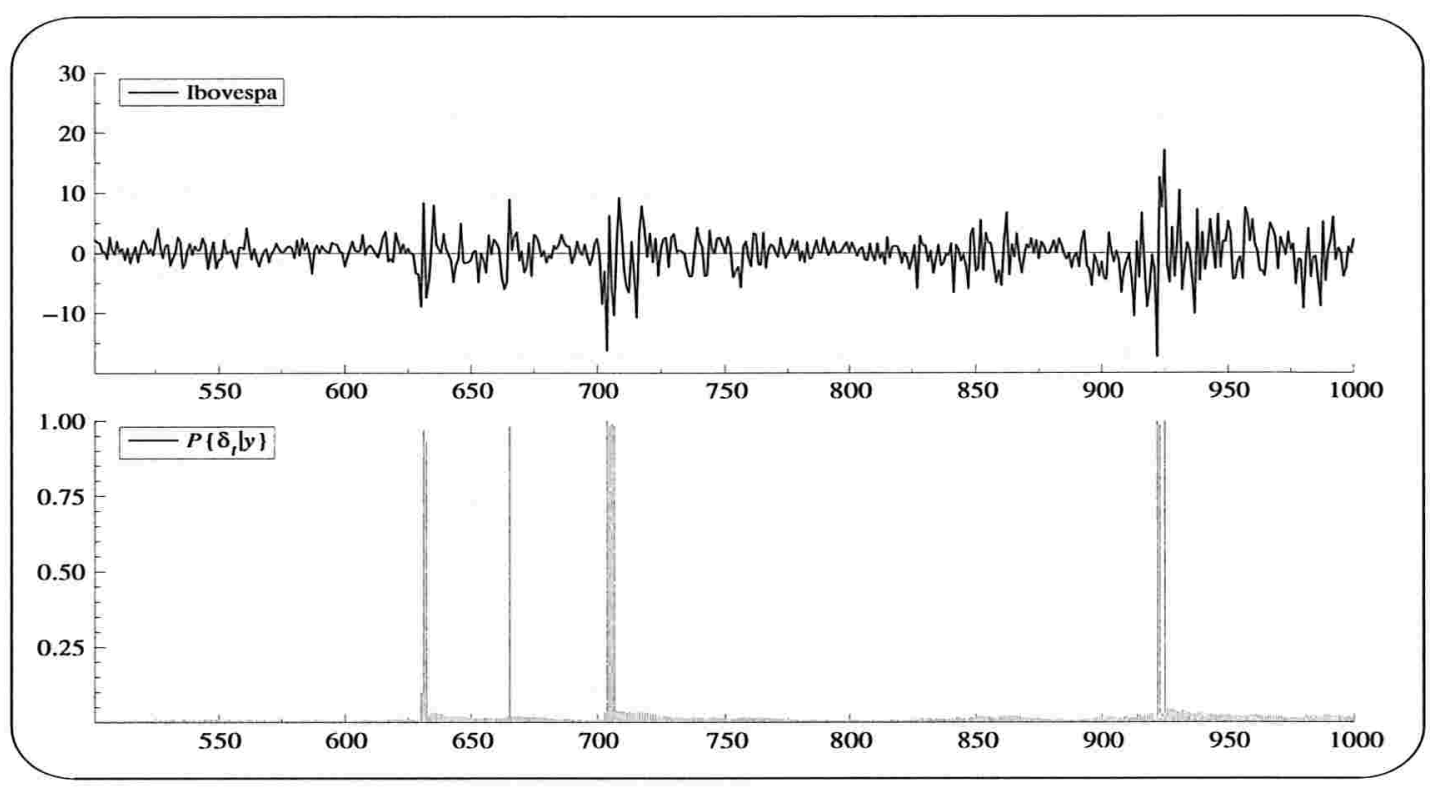

Figura 3.10: Outliers detectados na série Ibovespa: a) Retornos. b) Média da distribuição a posteriori do passo 7 (dentro dos blocos) e passo 1 (fora dos blocos) para detecção de outliers.

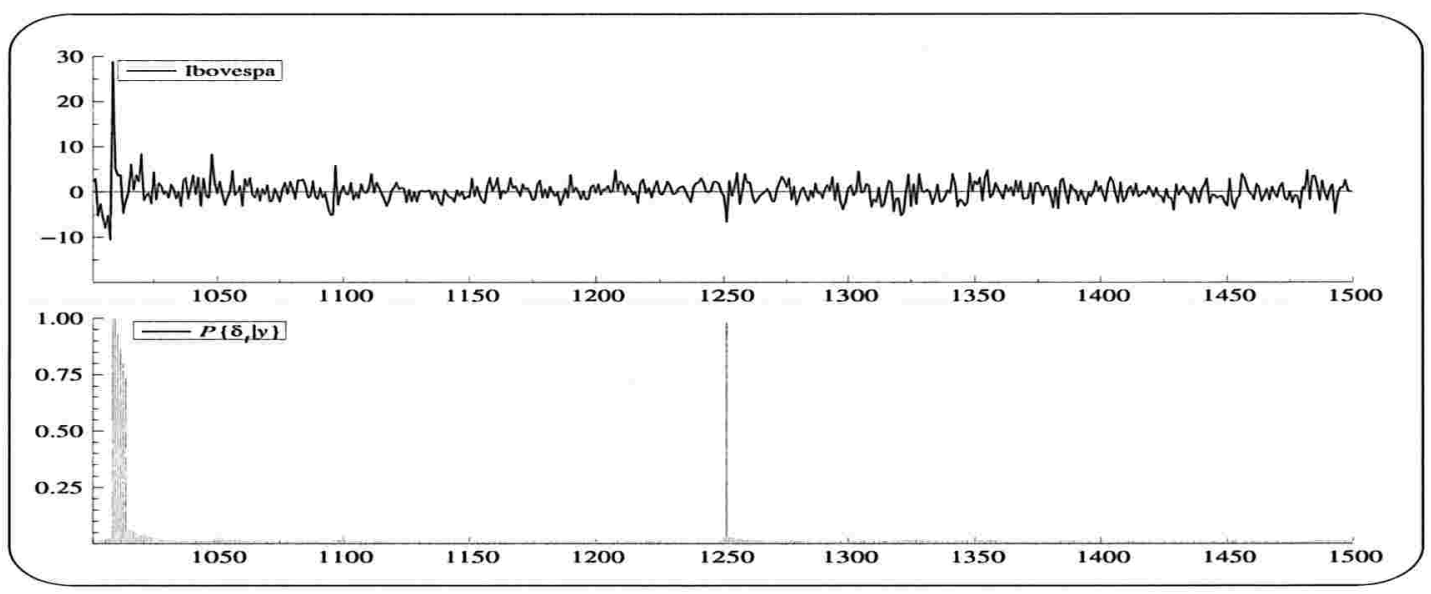

Figura 3.11: Outliers detectados na série Ibovespa: a) Retornos. b) Média da distribuição a posteriori do passo 7 (dentro dos blocos) e passo 1 (fora dos blocos) para detecção de outliers.

$\mathrm{dh}$ 
A Tabela 3.4 nos fornece os retornos detectados como outliers, sua data de ocorrência assim como sua localização nas figuras ilustrativas. A última coluna apresenta a probabilidade a posteriori do valor ser um outlier obtida através do amostrador de Gibbs Adaptado. São apresentadas apenas as observações que tiveram média das distribuição a posteriori da probabilidade de ser outlier maior do que 0.50 . Observe que as três menores probabilidades são iguais a $0.798,0.867$ e 0.933 , o que demonstra que as estimativas bayesianas das probabilidades ou são pequenas, menor do que 0.1 , ou grande.

\begin{tabular}{ccrc}
\hline \hline Data & Localização & Retorno & Probabilidade \\
\hline $10 /$ jan/1995 & 4 & $-10.47 \%$ & 0.9872 \\
$12 /$ jan/1995 & 6 & $9.22 \%$ & 0.9905 \\
$10 /$ mar/1995 & 45 & $22.72 \%$ & 1.0000 \\
$26 /$ out/1995 & 202 & $-6.84 \%$ & 0.9891 \\
$15 /$ jul/1997 & 629 & $-8.99 \%$ & 1.0000 \\
$16 /$ jul/1997 & 630 & $8.35 \%$ & 1.0000 \\
$17 /$ jul/1997 & 631 & $-7.58 \%$ & 0.9695 \\
$18 /$ jul/1997 & 632 & $-4.87 \%$ & 0.9334 \\
$27 /$ out/1997 & 703 & $-16.31 \%$ & 0.9840 \\
$28 /$ out/1997 & 704 & $6.14 \%$ & 0.9889 \\
$29 /$ out/1997 & 705 & $-6.30 \%$ & 0.9826 \\
$30 /$ out/1997 & 706 & $-10.42 \%$ & 0.9908 \\
$10 /$ set/1998 & 921 & $-17.32 \%$ & 1.0000 \\
$11 /$ set/1998 & 922 & $12.49 \%$ & 0.9861 \\
$15 /$ set/1998 & 924 & $17.04 \%$ & 1.0000 \\
$14 /$ jan/1999 & 1007 & $-10.59 \%$ & 1.0000 \\
$15 /$ jan/1999 & 1008 & $28.73 \%$ & 1.0000 \\
$18 /$ jan/1999 & 1009 & $5.21 \%$ & 0.9352 \\
$19 /$ jan/1999 & 1010 & $3.59 \%$ & 0.8667 \\
$20 /$ jan/1999 & 1011 & $3.81 \%$ & 0.7983 \\
$04 /$ jan/2000 & 1250 & $-6.67 \%$ & 0.9810 \\
\hline \hline
\end{tabular}

Tabela 3.4: Outliers identificados no Ibovespa.

Podemos verificar através da Tabela 3.4 que das 21 observações que o método detectou como outlier, 14 estão relacionadas com alguma crise. Por exemplo, no período de 10 de março de 1995 foi o período de introdução de intrabandas cambiais e entre 15 e 18 de julho de 1997 foi período da primeira crise da Ásia. Em 15 de setembro de 1998 tivemos uma crise mundial, quando as bolsas em todo o mundo 
despencaram e houve aumento generalizado das taxas de juros domésticas. Durante meados do mês de janeiro de 1999 tivemos a desvalorização do real. Neste período, de 14 de janeiro de 1999 a 20 de janeiro tivemos 5 observações coonsecutivas com probabilidade a posteriori decrescente. Estes valores são típicas influencia de um IO, indicando que a desvalorização do real levou a uma aumento de volatilidade.

Apesar da maioria das observações detectadas estarem relacionadas com crises, pode-se verificar que o método não detectou a moratória da Rússia em agosto de 1998. 


\subsubsection{Análise da Série da NYSE}

Na série de retornos do NYSE, completamos o modelo especificando as distribuições a posterioris análogo ao que fizemos para o caso do índice Bovespa.

A Figura 3.12 nos fornece as estimativas dos parâmetros para o modelo. Apresentamos as últimas 20000 iterações para visualizarmos a distribuição a posteriori do parâmetros.

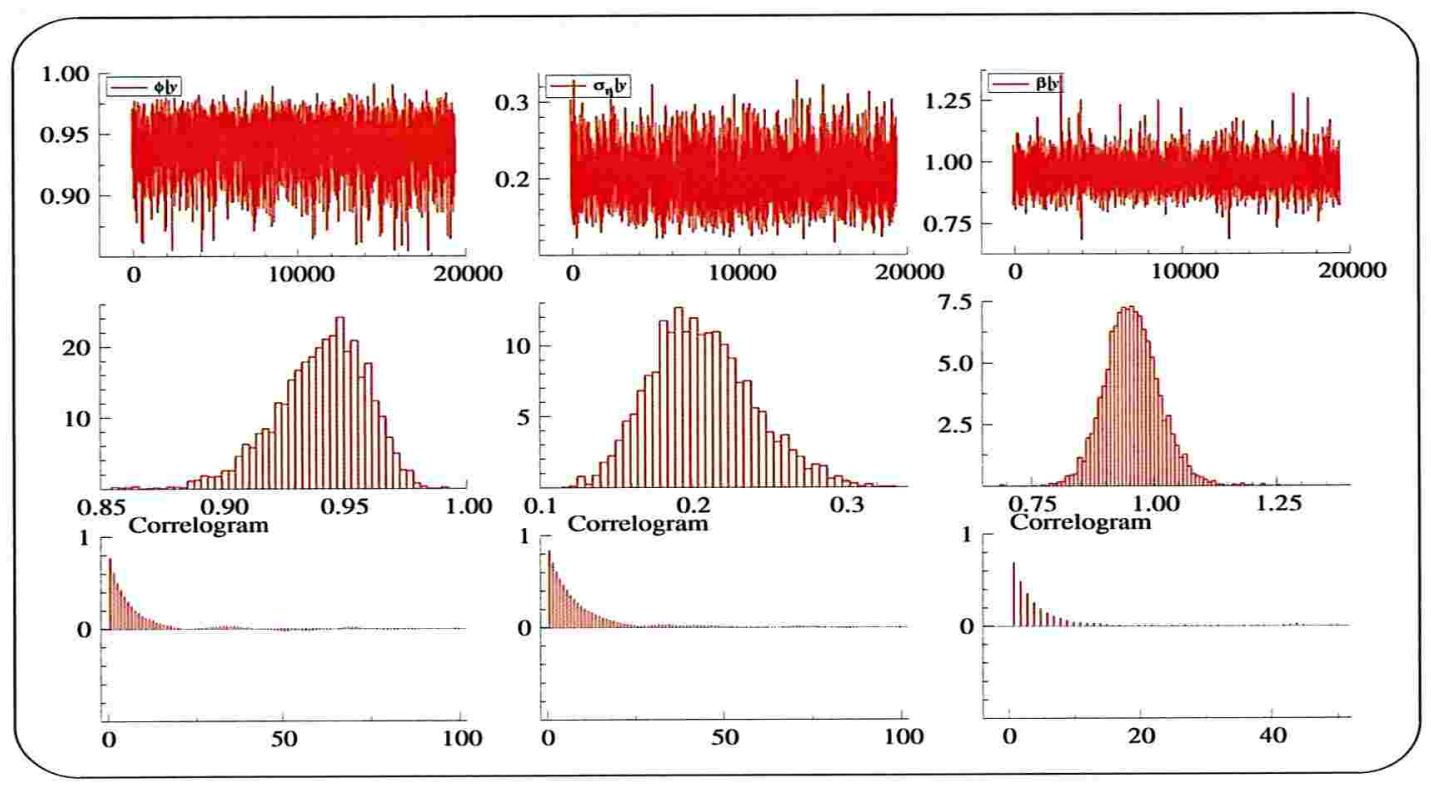

Figura 3.12: Gráficos dos valores amostrados, histogramas das densidades posterioris dos parâmentros e correlograma para amostragem da série da NYSE. 
A Tabela 3.5 apresenta as estimativas dos parâmetros e seus respectivos desvios. Nota-se que as estimativas dos parâmetros são relativamente próximas quando consideramos o amostrador de Gibbs padrão e o adaptado.

\begin{tabular}{c|cc}
\hline \hline \multirow{2}{*}{ Parâmetros } & $\begin{array}{c}\text { GS } \\
\text { Padrão }\end{array}$ & $\begin{array}{c}\text { GS } \\
\text { Adaptado }\end{array}$ \\
\hline$\phi$ & 0.9284 & 0.9398 \\
$(0.0047)$ & $(0.0156)$ \\
$\sigma_{\eta}$ & 0.2154 & 0.2131 \\
$\beta$ & $(0.0089)$ & $(0.0121)$ \\
$\beta$ & 1.2414 & 1.1503 \\
$(0.0913)$ & $(0.0857)$ \\
\hline \hline
\end{tabular}

Tabela 3.5: Média e desvios das distribuições a posteriori dos parâmetros do modelo para NYSE.

As Figuras 3.13, 3.14 e 3.15 apresentam as estimativas da probabilidade posteriori de um determinado valor ser um outlier dadas nos passos 1 e 7 . A distribuição a posteriori ilustrada é obtida através do Amostrador de Gibbs Adaptado, ou seja, a partir da amostragem conjunta dos blocos de outliers.

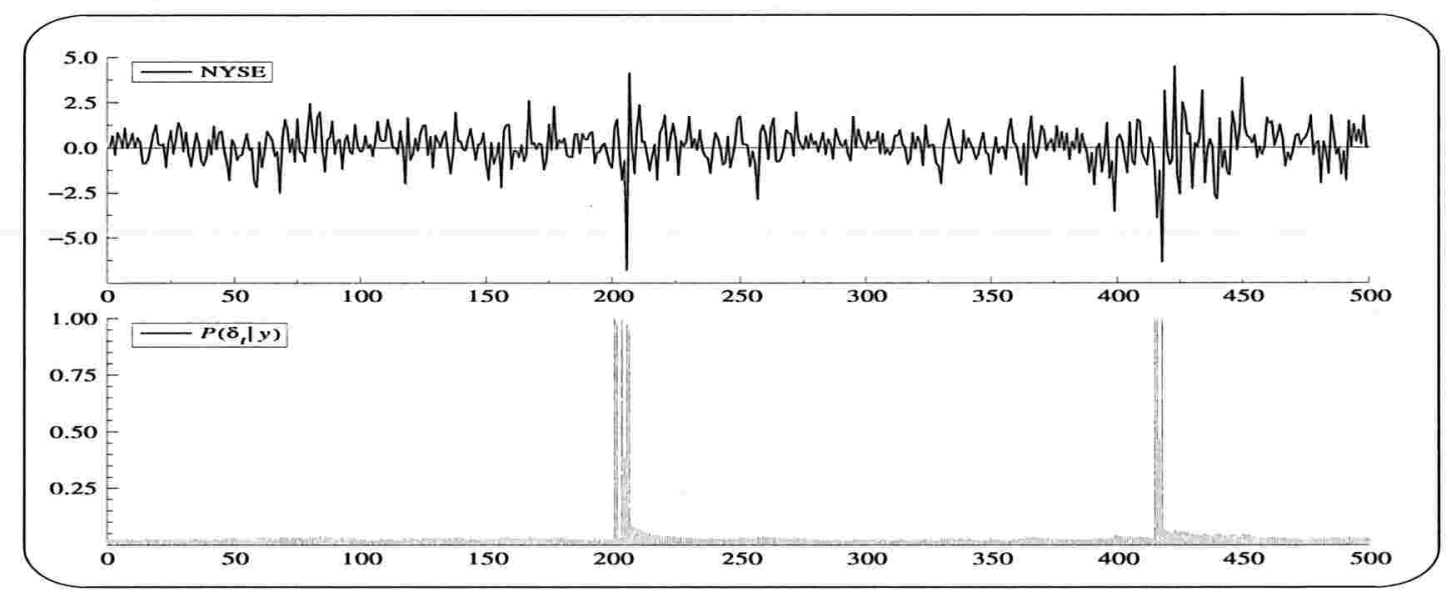

Figura 3.13: Outliers detectados na série NYSE primeiras 500 observações: a) Retornos. b) Média da distribuição a posteriori do passo 7 (dentro dos blocos) e passo 1 (fora dos blocos) para detecção de outliers. 


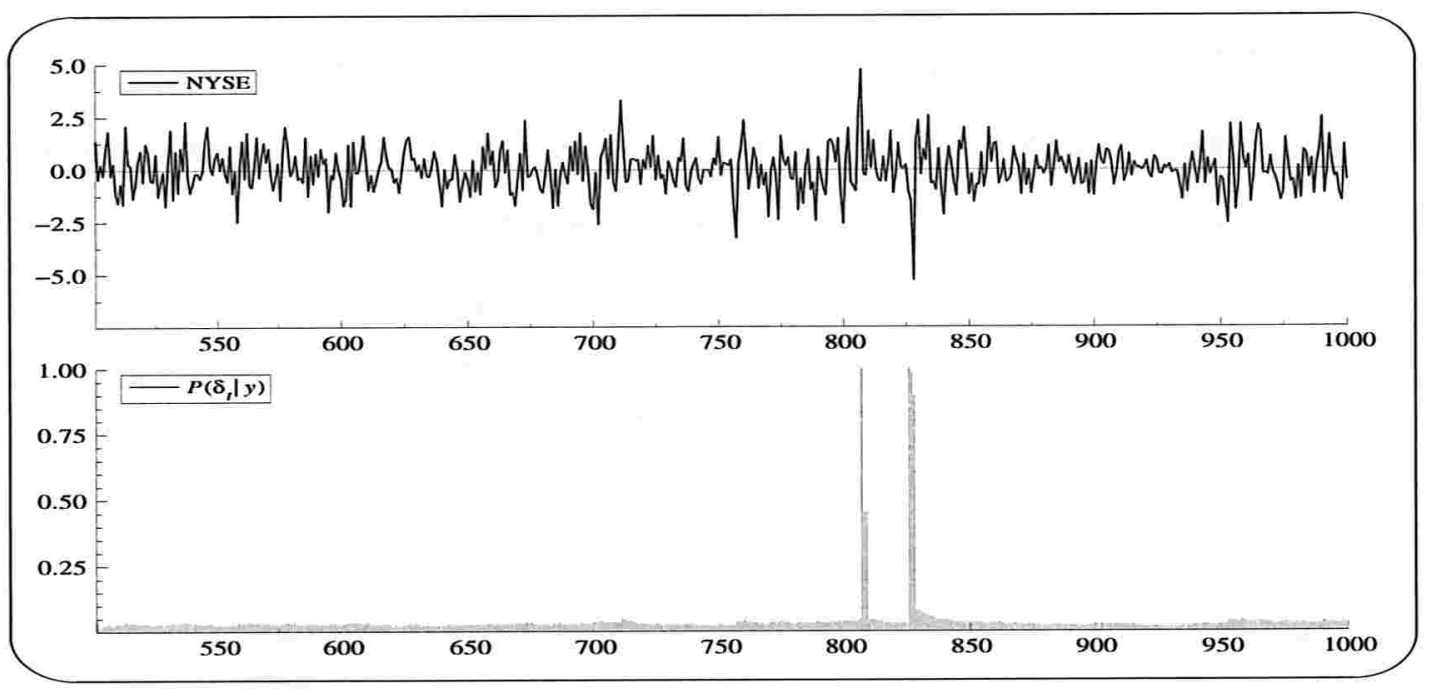

Figura 3.14: Outliers detectados na série NYSE entre observação 501 e 1000: a) Retornos. b) Média da distribuição a posteriori do passo 7 (dentro dos blocos) e passo 1 (fora dos blocos) para detecção de outliers.

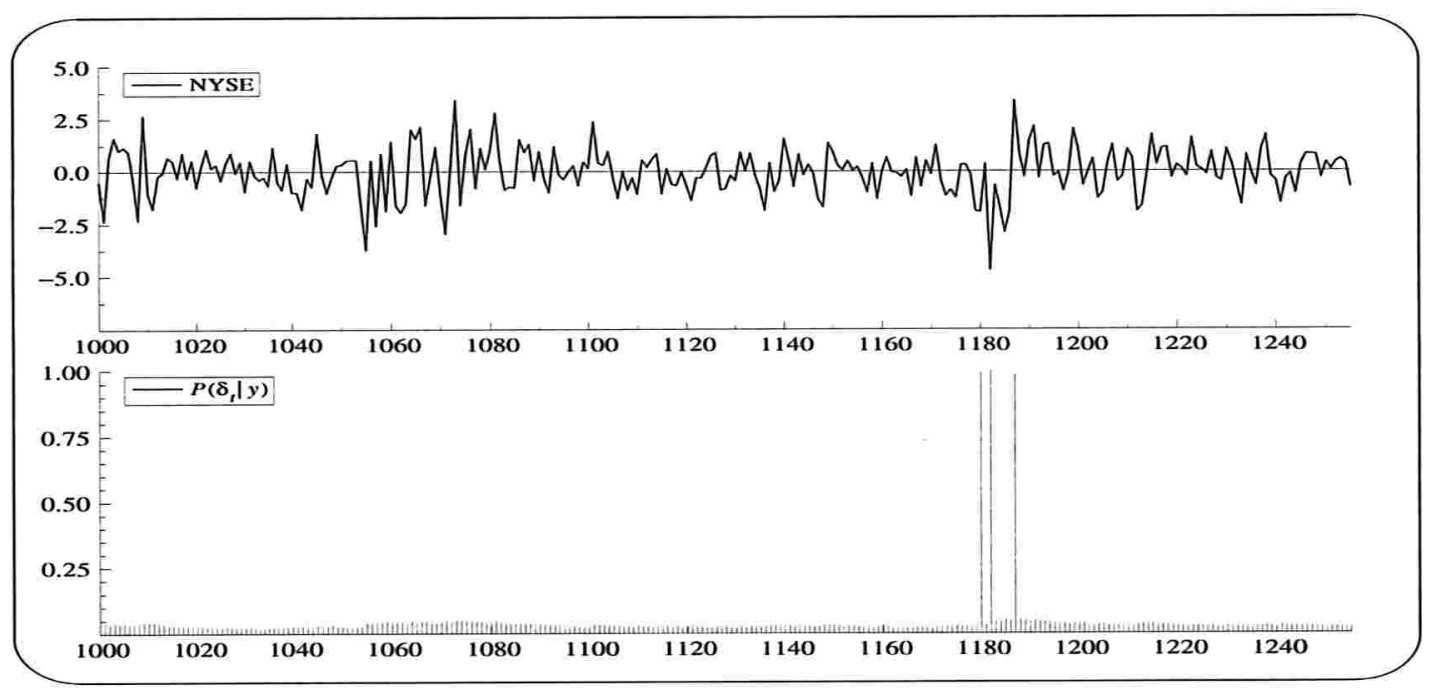

Figura 3.15: Outliers detectados na série NYSE entre observação 1000 e 1255: a) Retornos. b) Média da distribuição a posteriori do passo 7 (dentro dos blocos) e passo 1 (fora dos blocos) para detecção de outliers. 
A Tabela 3.6 nos fornece os retornos detectados como outliers (probabilidade $i$ 0.5), sua data de ocorrência assim como sua localização nas figuras ilustrativas. A última coluna apresenta a probabilidade a posteriori do valor ser um outlier obtida através do amostrador de Gibbs Adaptado. No entanto, as probabilidades ou são altas ou muito baixas. Exceto por três observações com probabilidade no intervalo $(0.3 ; 0.5)$, elas são grandes (ver Tabela 3.6 ) ou menores do que 0.1 , mostrando que o método detecta claramente a maioria das observações.

\begin{tabular}{ccrc}
\hline \hline Data & Localização & Retorno & Média Posteriori \\
\hline $20 / 10 / 1997$ & 201 & $1.139 \%$ & 1.0000 \\
$21 / 10 / 1997$ & 202 & $1.567 \%$ & 0.9811 \\
$23 / 10 / 1997$ & 204 & $-1.847 \%$ & 0.9939 \\
$27 / 10 / 1997$ & 206 & $-6.791 \%$ & 0.9745 \\
$28 / 10 / 1997$ & 207 & $4.113 \%$ & 0.9556 \\
$27 / 08 / 1998$ & 415 & $-1.005 \%$ & 1.0000 \\
$28 / 08 / 1998$ & 416 & $-3.920 \%$ & 1.0000 \\
$01 / 09 / 1998$ & 418 & $-6.352 \%$ & 1.0000 \\
$16 / 03 / 2000$ & 807 & $4.748 \%$ & 1.0000 \\
$12 / 04 / 2000$ & 826 & $-1.056 \%$ & 1.0000 \\
$13 / 04 / 2000$ & 827 & $-1.484 \%$ & 0.9852 \\
$14 / 04 / 2000$ & 828 & $-5.275 \%$ & 0.8976 \\
$07 / 09 / 2001$ & 1180 & $-1.948 \%$ & 0.9771 \\
$17 / 09 / 2001$ & 1182 & $-4.701 \%$ & 0.9984 \\
$24 / 09 / 2001$ & 1187 & $3.356 \%$ & 0.9642 \\
\hline \hline
\end{tabular}

Tabela 3.6: Outliers identificados na NYSE e suas respectivas média posteriori da probabilidade de ser um outlier para observações com probabilidade a posteriori maior que 0.5 .

A Tabela 3.6 apresenta as observações detectadas como outliers, podemos verificar que das 15 observações apenas 5 não estão relacionadas diretamente à crises conhecidas.

As observações do período entre 20 a 28 de outubro de 1997 estão relacionadas 
com a segunda crise da Ásia, no fim de agosto de 1998 detectamos a moratória da Rússia, lembre que o método não detectou essa crise na análise da série de retornos do Ibovespa. A crise da Nasdaq foi detectada pelo método e ocorreu em na segunda quinzena de abril de 2000 .

\subsection{Conclusões}

Neste capítulo verificamos a eficiência do tratamento bayesiano quando consideramos a detecção de outliers. Constatamos que a metodologia é robusta para a detecção de valores atípicos quando esses ocorrem isolados ou em blocos. As simulações mostraram que os valores considerados outliers são detectados com alta probabilidade a posteriori. As análises das séries reais mostraram-se consistentes com os resultados obtidos através de outras metodologias existentes na literatura. É interessante notar que durante as crises da Ásia (outubro de 1997), Rússia (agosto de 1998), Nasdaq (Abril de 2000) e o ataque terrorista de 11 de setembro de 2001, temos outliers detectados. Ao contrário do Ibovespa, a primeira crise da Ásia e a crise cambial do Brasil não tiveram os efeitos detectados. Uma comparação com outros métodos, e no caso da série do NYSE, uma comparação com os resultados de Zhang e King (2005) serão apresentadas no capítulo 6 . 


\section{CAPÍTULO 4}

\section{Estatística de Influência em Modelos de Volatilidade}

Neste capítulo apresentaremos uma estatística para diagnosticar influência em modelos de volatilidade estocástica baseada no trabalho de Peña (2005). Na seção 4.1 apresentaremos o conceito de análise de influência e na seção 4.2 é apresentada a estatística para diagnosticar influência em modelo de volatilidade estocástica. A seção 4.3 considera o estudo das propriedades da estatística proposta, e finalmente na seção 4.4 analisamos a eficiência do método utilizando as séries simuladas e aplicamos o algoritmo proposto às séries do Ibovespa e NYSE. 


\subsection{Análise de Influência}

Os modelos estatísticos são amplamente utilizados para extrair e entender as características importantes de um conjunto de dados. Por outro lado, sabemos que os modelos são aproximações de processos subjacentes e, portanto, nunca são perfeitos. Uma compreensão e modelagem adequadas dependem fortemente do entendimento da importância de uma observação ou de um conjunto de observações. O artigo de Cook (1977) relativo ao estudo de diagnóstico de modelos e outliers teve uma forte influência na literatura. Essa influência foi maior na aplicação a modelos lineares (ver, por exemplo, o livro de Cook e Weisberg, 1982), mas se estendeu para outros tipos de modelos.

A influência pode ser definida, informalmente, como sendo o efeito de modificações de grupos de observações, ou de uma única observação, em saídas de um modelo. A influência pode ser medida, por exemplo, através do efeito nas previsões, estimativas, verossimilhança. Como forma de modificações podemos ter desde a simples exclusão de observações, como proposta por Cook (1977), mudanças de pesos das modificações, como proposta por Belsley et al.(1980), ou perturbações em direções específicas como na análise de influência local proposta por Cook (1986). Várias abordagens similares à distância de Cook foram propostas na literatura (ver, Cook et al., 1988), mas muitas delas sofrem o problema de mascaramento na presença de conjunto de pontos influentes. Peña (2005) introduziu uma nova forma de medir influência em modelos de regressão linear que diminui este problema. Em vez de verificar como a deleção de uma observação influencia estimativas dos parâmetros, previsões ou verossimilhança, ele verifica como um ponto é influenciado pelos outros pontos da amostra.

Neste capítulo verificamos como este mesmo tipo de abordagem pode ser aplicada 
a modelos de volatilidade estocástica. Na subseção 4.2 apresentamos inicialmente a abordagem de Cook (1977), a nova proposta de Peña (2005), sua adaptação aos modelos de volatilidade estocástica e os algoritmos necessários para a sua aplicação. Nos modelos de regressão linear, Peña (2005) analisa algumas das propriedades da estatística proposta, propriedades estas que não são necessariamente verdadeiras para os modelos de volatilidade estocástica. As propriedades da estatística no modelo de volatilidade estocástica são derivadas através de simulação na seção 4.3. Como sua distribuição não depende fortemente dos parâmetros verdadeiros podemos utilizar os parâmetros estimados para estimar a distribuição da estatística proposta. Finalmente, aplicamos o método na subseção 4.4 para séries simuladas e reais apresentadas no capítulo 2.

Neste capítulo apresentaremos inicialmente a extensão do trabalho de Peña (2005) ao modelo de volatilidade estocástica. Posteriormente serão apresentadas algumas propriedades empíricas distribuicionais através de estudos de simulação de algumas séries financeiras. Finalmente o método será aplicado à série do Indice Bovespa e para os dados da NYSE. 


\subsection{Estatística para Diagnosticar Influência}

O artigo de Cook (1977) apresentou uma nova abordagem para as investigações de análise de valores espúrios e diagnóstico de modelos estatísticos. Antes da proposta de Cook as pesquisas relacionadas ao tratamento de observações discrepantes estavam voltadas à análise, principalmente gráfica, dos resíduos, $R_{i}$; em particular, os resíduos padronizados e resíduos Studentizados.

Cook (1977) propôs uma medida de simples interpretação de forma a combinar as informações fornecidas pela variância dos resíduos $\left(V\left(R_{i}\right)\right)$ e as informações fornecidas pelo resíduo Studentizado. O objetivo era caracterizar os valores considerados críticos, através da análise de influência de uma determinada observação na estimativa do conjunto de parâmetros, em um modelo de regressão linear.

Cook determinou uma medida da distância entre estimativa dos parâmetros através do conceito de elipsóide de confiança para os parâmetros do modelo. Foram consideradas todas as observações, $\hat{\beta}$ e a estimativa dos parâmetros quando excluimos a $i$-ésima observação, $\hat{\beta}_{(-i)}$.

Considere o modelo de regressão dado por

$$
\mathbf{Y}=\mathbf{X} \beta+\epsilon
$$

onde $\mathbf{Y}$ é um vetor de dimensão $n \times 1$ de observações, $\mathbf{X}$ é uma matriz de dimensão $n \times p$ e de posto $p$ completo, $\beta$ é um vetor $n \times p$ de parâmetros e $\epsilon$ é um vetor aleatório com média zero e variância $\sigma_{\epsilon}^{2} I_{n \times n}$, onde $I_{n \times n}$ é a matriz identidade de ordem $n$. Cook definiu uma medida de distância entre $\hat{\beta}$ e $\hat{\beta}_{(-i)}$ como 


$$
D_{i}=\frac{\left(\hat{\beta}_{(-i)}-\hat{\beta}\right)^{\prime} \mathbf{X}^{\prime} \mathbf{X}\left(\hat{\beta}_{(-i)}-\hat{\beta}\right)}{p s^{2}}=\frac{\left\|\hat{\mathbf{y}}-\hat{\mathbf{y}}_{(i)}\right\|^{2}}{p s^{2}}
$$

onde $\hat{\mathbf{y}}_{(i)}$ é o preditor de $y$ utilizando a estimativa $\hat{\beta}_{(-i)}$ e $s^{2}=\mathbf{R}^{\prime} \mathbf{R} /(n-p)$ e $i=$ $1, \ldots, n$. Tal medida pode ser reescrita como:

$$
D_{i}=\frac{t_{i}^{2}}{p} \frac{V\left(\hat{Y}_{i}\right)}{V\left(R_{i}\right)}
$$

onde $V\left(\hat{Y}_{i}\right)=\mathbf{x}_{i}^{\prime}\left(\mathbf{X}^{\prime} \mathbf{X}\right)^{-1} \mathbf{x}_{i} \sigma_{\epsilon}^{2}$ e $V\left(R_{i}\right)=\sigma_{\epsilon}^{2}\left[1-\mathbf{x}_{i}^{\prime}\left(\mathbf{X}^{\prime} \mathbf{X}\right)^{-1} \mathbf{x}_{i}\right]$ e $t_{i}$ são os resíduos Studentizados, dados por:

$$
t_{i}=\frac{Y_{i}-\mathbf{x}_{\mathbf{i}}^{\prime} \hat{\beta}}{s \sqrt{1-\mathbf{x}_{i}^{\prime}\left(\mathbf{X}^{\prime} \mathbf{X}\right)^{-1} \mathbf{x}_{i}}},
$$

onde $\mathbf{x}_{\mathbf{i}}{ }^{\prime}$ é a $i$-ésima linha de $\mathbf{X}$. Assim vemos, pela relação dada na Equação (4.2.1), que a quantidade $t_{i}$ captura a discrepância da $i$-ésima observação em relação ao modelo subjacente. Por outro lado, o quociente de variâncias nos fornece o quanto a $i$ ésima observação influencia na estimativa dos parâmetros. Assim, a Equação (4.2.1) produz uma medida do impacto de um determinado ponto na solução de mínimos quadrados.

Várias abordagens similares à esta distância foram propostas na literatura onde é analisada a influência de uma ou mais observações nas estimativas dos parâmetros, previsões ou da função de verossimilhança. Peña (2005) introduziu uma nova forma de analisar influência, considerando como uma determinada observação ou valor é influenciado pelas outras observações da amostra. O procedimento verifica como a predição da $i$-ésima observação é influenciada ao re-estimarmos esse ponto considerando a ausência de cada uma das demais observações. Portanto, Peña mede 
as variações na previsão ao considerar cada uma das observações como falta de informação. Vamos evitar o termo excluir, porque em séries temporais isto tem outro efeito. Verifica-se que tal procedimento é mais eficiente para indicar características nos dados tais como conjuntos (cluster) de outliers que são difíceis de serem detectados através de estatísticas de influência usuais.

A estatística baseia-se em medir como a exclusão de cada observação afeta a previsão de uma observação em particular. Desta forma considera-se como cada observação está sendo influenciada pelo resto dos dados. Peña definiu a estatística como a norma ao quadrado do vetor $\mathbf{s}_{\mathbf{i}}=\left(\hat{y}_{i}-\hat{y}_{i(1)}, \ldots, \hat{y}_{i}-\hat{y}_{i(n)}\right)^{\prime}$, isto é,

$$
S_{i}=\frac{\mathbf{s}_{\mathbf{i}} \mathbf{s}_{\mathbf{i}}}{p \hat{\operatorname{Var}}\left(\hat{y}_{i}\right)}
$$

e apresentou três propriedades da estatística proposta. A primeira propriedade é que sob algumas hipóteses, a estatística possui média igual ao inverso do posto da matrix $\mathbf{X}$ no modelo de regressão. A segunda propriedade é que assintóticamente a estatística $S_{i}$ é aproximadamente normal. Por último, Peña verificou que quando a amostra está contaminada por grupos de outliers, a estatística discrimina os outliers das demais observações. 


\subsubsection{Estatística para Modelo de Volatilidade Estocástica}

Considere sem perda de generalidade o modelo de volatilidade estocástica dado por;

$$
\begin{aligned}
& y_{t}=\beta e^{h_{t} / 2} \varepsilon_{t}, \quad t \geqq 1, \\
& h_{t}=\phi h_{t-1}+\sigma_{\eta} \eta_{t} .
\end{aligned}
$$

Denote por $\mathbf{y}=\left(y_{1}, \ldots, y_{n}\right)^{\prime}$ o vetor completo de observações e por $\hat{\Psi}=\left(\beta, \hat{\phi}^{\prime}, \hat{\sigma}_{\eta}\right)$ o vetor das estimativas dos parâmetros baseada em todas as observações. Para obtermos linearidade da equação acima, tomamos uma transformação logaritmo do quadrado:

$$
\log \left(y_{t}^{2}\right)=h_{t}+\log \left(\varepsilon_{t}^{2}\right)
$$

Dado que $\varepsilon_{t}$ é normalmente distribuído com $E\left[\varepsilon_{t}\right]=0$ e $\operatorname{Var}\left[\varepsilon_{t}\right]=1$ então $\log \left(\varepsilon_{t}^{2}\right)$ segue a distribuição log-qui-quadrado com média -1.27 e variância $\pi^{2} / 2$. Considerando $\xi_{t}=\log \left(\varepsilon_{t}^{2}\right)-E\left[\log \left(\varepsilon_{t}^{2}\right)\right]=\log \left(\varepsilon_{t}^{2}\right)+1.27$, as equações em (4.2.1) serão modificadas para:

$$
\begin{aligned}
\log \left(y_{t}^{2}\right) & =-1.27+h_{t}+\xi_{t} & \xi_{t} & \sim \operatorname{ID}\left(0, \frac{\pi^{2}}{2}\right), \\
h_{t} & =\phi h_{t-1}+\eta_{t} & \eta_{t} & \sim \operatorname{NID}\left(0, \sigma_{\eta}^{2}\right) .
\end{aligned}
$$

Considere $y_{t}^{*}=\log \left(y_{t}^{2}\right)-1.27$ para simplificarmos a notação. Uma vantagem desta transformação é que, em geral, para as séries financeiras empíricas a distribuição de $\log \left(y_{t}^{2}\right)$ é mais simétrica do que a distribuição de $y_{t}$. Porém o coeficiente de simetria é ligeiramente negativo.

Agora, consideremos o procedimento onde faremos a predição da $i$-ésima observação $n$ vezes. Esta será prevista considerando como faltante cada uma das $n$ 
observações e reestimamos os parâmetros. Dessa forma, considerando que $\mathbf{y}_{(i)}$ é o vetor de observações considerando o $i$-ésimo ponto como faltante, teremos que a estimativa do conjunto de parâmetros baseada em $\mathbf{y}_{(i)}$ será representada por $\hat{\Psi}_{(i)}=$ $\left(\hat{\beta}_{(i)}, \hat{\phi}_{(i)}, \hat{\sigma}_{\eta(i)}\right)^{\prime}$, com $i=(1, \ldots, n)$.

A estatística baseia-se em medir o efeito da consideração de cada observação como faltante na previsão de uma observação em particular. Assim, considera-se como cada observação está sendo influenciada pelo resto dos dados. Definimos a estatística como a norma ao quadrado do vetor $\mathbf{s}_{\mathbf{i}}=\left(\hat{y}_{i}-\hat{y}_{i(1)}, \ldots, \hat{y}_{i}-\hat{y}_{i(n)}\right)^{\prime}$, isto é,

$$
S_{i}=\frac{\mathbf{s}_{\mathbf{i}}{ }^{\prime} \mathbf{s}_{\mathbf{i}}}{\hat{\operatorname{Var}}\left(\hat{y}_{i}\right)} \quad i=1, \ldots, n \text {. }
$$

O procedimento pode ser resumido pelo seguinte algoritmo:

1. Estime os parâmetros, $\Psi$, do modelo de volatilidade.

2. Faça $i=1, \ldots, n$

i. Considere a estimativa, $\hat{h}_{i}$ considerando $\hat{\Psi}$.

ii. Faça $j=1, \ldots, n$

- Trate a $j$-ésima observação como valor faltante, obtendo $\mathbf{y}_{(j)}$.

- Reestime o modelo obtendo-se $\Psi_{(j)}$.

- Encontre $\hat{h}_{i(j)}$, a estimativa de $h_{i}$ baseada em $\mathbf{y}_{(j)}$.

3. Calcule a Estatística $S_{i}$.

4. Calcule a Estatística $S^{*} \underset{1 \leq i \leq T}{\max } S_{i}$. 
Temos, portanto, para cada série as estatísticas $S^{*}$ e a seqüência $\left\{S_{i}\right\}_{1 \leq i \leq T}$. A primeira estatística pode ser utilizada como um teste geral e as outras como testes para cada observação.

Os parâmetros do modelo de volatilidade da Equação (4.2.1), $\Psi=\left(\beta, \phi, \sigma_{\eta}\right)$ e as volatilidades, serão estimados através da quasi-verossimilhança a fim de diminuir o tempo computacional que seria bem superior se considerássemos as estimativas dos parâmetros pela verossimilhança exata. Isto torna-se mais importante quando utilizarmos simulação Monte Carlo para encontrar a distribuição de $S^{*}$.

As estimativas de quasi-verossimilhança de $\hat{h}_{i}$ e $\hat{h}_{(i)}$, assim como a variância de $\hat{y}_{t}$, foram obtidas através do algoritmo implementado no SsfPack proposto por Koopman et al. (1999). Para a maximização da quasi-verossimilhança será utilizado o método numérico BFGS implementado na linguagem Ox. 


\subsection{Propriedades da Estatística para o Modelo de Volatilidade}

Nesta seção estudaremos a distribuição da estatística proposta através de simulação Monte Carlo. Inicialmente serão verificadas algumas características distribucionais da estatística proposta para modelos de volatilidade estocástica sem a introdução intencional de valores influentes ou outliers. Caso estas distribuições sejam pouco sensíveis a valores dos parâmetros verdadeiros podemos estimar as distribuições de referência simulando através de modelos com parâmetros estimados para testar as hipóteses de interesse. Posteriormente, serão incluídos valores aberrantes isolados ou em conjuntos para verificar se a estatística tem poder de detectar estas observações.

\subsubsection{Distribuição de Referência e Poder}

Considere que estamos interessados em estimar a distribuição da estatística quando o processo segue um processo de volatilidade estocástica com parâmetros fixos e conhecidos, $\Psi_{0}$, para um determinado tamanho da amostra $T$. Esta distribuição pode ser estimada através de simulação Monte Carlo gerando-se um número suficientemente grande de séries e calculando-se as estatísticas. Na prática, entretanto, não conhecemos o valor verdadeiro dos parâmetros e uma alternativa natural é utilizar o valor dos parâmetros estimados. Como toda estimativa está sujeita a erros, este procedimento não pode ser utilizado, se a distribuição da estatística depender fortemente dos parâmetros. Desta forma, iremos inicialmente explorar as características distribucionais da estatística proposta, sendo a sensibilidade uma das mais importantes. 
Como nas aplicações empíricas serão consideradas as séries do Ibovespa e NYSE utilizaremos na simulação os valores dos parâmetros estimados para estas séries e perturbações em torno delas. Para cada modelo serão realizadas 3000 replicações. Os valores dos parâmetros e os tamanhos das amostras serão especificadas na apresentação das análises específicas.

\subsubsection{Análise de Sensibilidade}

$\mathrm{Na}$ análise de sensibilidade das distribuições não é tão importante realizar as simulações para vários tamanhos da amostra. Desta forma, escolhemos o tamanho da amostra igual a 1000 e foram utilizados os valores dos parâmetros dados na Tabela 4.1. Os dois primeiros valores são relativos à serie do Ibovespa e os dois últimos à série do NYSE. No capítulo 2 os valores estimados de $\phi$ e $\sigma_{\eta}$ pelo método da quasiverossimilhança foram iguais a $\Psi_{I B_{1}}$ e $\Psi_{N Y_{1}}$ respectivamente. Na série real estimada no Capítulo 2 os valores de $\phi$ e $\sigma_{\eta}^{2}$ foram estimados com desvio padrão iguais a $(0,011$; $0,055)$ e $(0,013 ; 0,009)$ para Ibovespa e NYSE respectivamente. Desta forma foram consideradas perturbações aproximadamente iguais a $(1 ; 1,15)$ desvios e $(1,5 ; 1,3)$ desvios para as séries do Ibovespa e NYSE, respectivamente. 
$\mathrm{Na}$ análise a ser apresentada apenas consideraremos a distribuição de $S^{*}$ considerando um total de 3000 replicações. As Figuras 4.1 e 4.2 apresentam os histogramas das distribuições dos máximos e, como estamos mais interessados na cauda superior as Figuras 4.3 e 4.4 apresentam a distribuição empírica nesta cauda. O parâmeto ( $\beta$ ou $\mu$ ) é de escala e não é importante na distribuição da estatística do teste.

\begin{tabular}{||c||cc||}
\hline \hline \multicolumn{1}{|c||}{} & \multicolumn{2}{c||}{ Parâmetros } \\
\hline Modelos & $\phi$ & $\sigma_{\eta}^{2}$ \\
\hline$\Psi_{I B_{1}}$ & 0.9811 & 0.0361 \\
$\Psi_{I B_{2}}$ & 0.9700 & 0.0289 \\
$\Psi_{N Y_{1}}$ & 0.9310 & 0.0324 \\
$\Psi_{N Y_{2}}$ & 0.9500 & 0.0441 \\
\hline \hline
\end{tabular}

Tabela 4.1: Valores para o conjunto de parâmetros das séries.

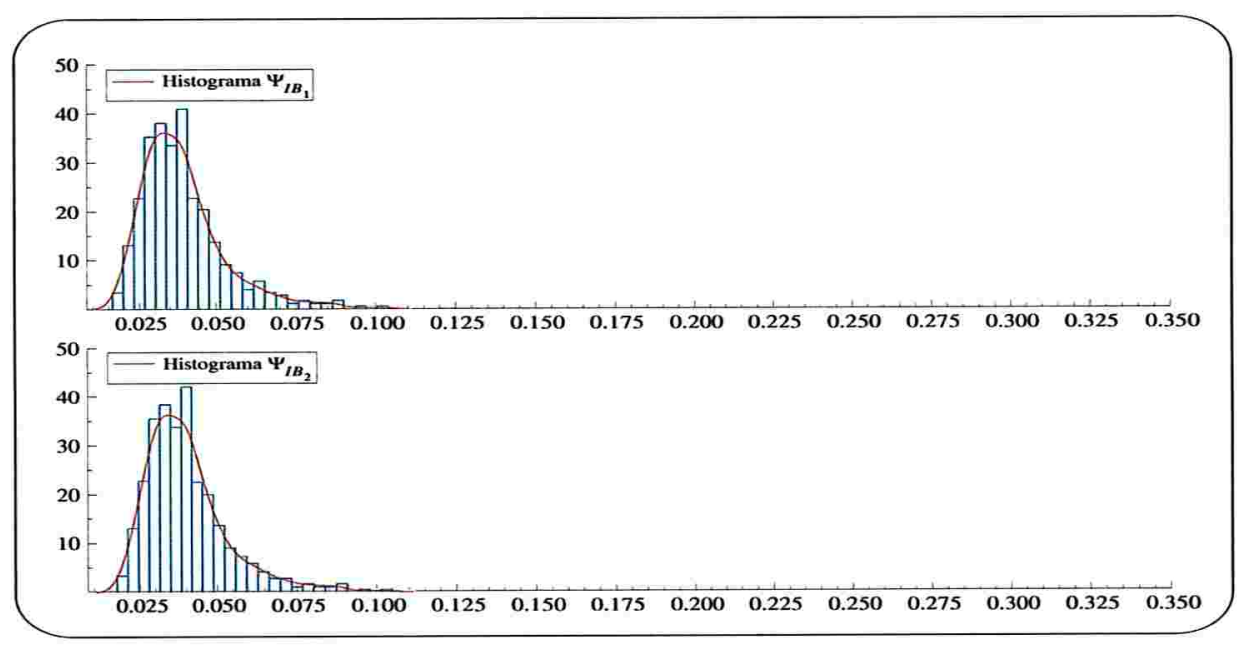

Figura 4.1: Histograma da distribuição dos máximos, $S^{*}$, baseada em 3000 replicações, tamanho da série igual a 1000 e conjunto de parâmetros $\Psi_{I B_{1}}$ e $\Psi_{I B_{2}}$. 


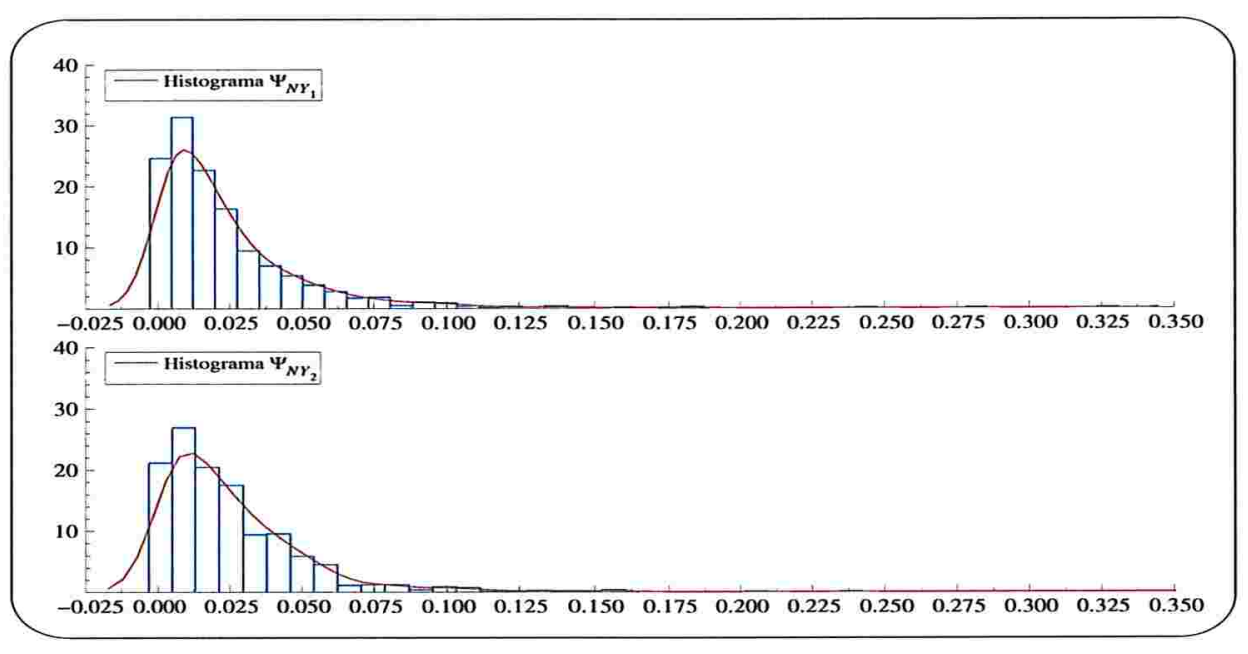

Figura 4.2: Histograma da distribuição dos máximos baseada em 3000 replicações, tamanho da série igual a 1000 e conjunto de parâmetros $\Psi_{N Y_{1}}$ e $\Psi_{N Y_{2}}$.

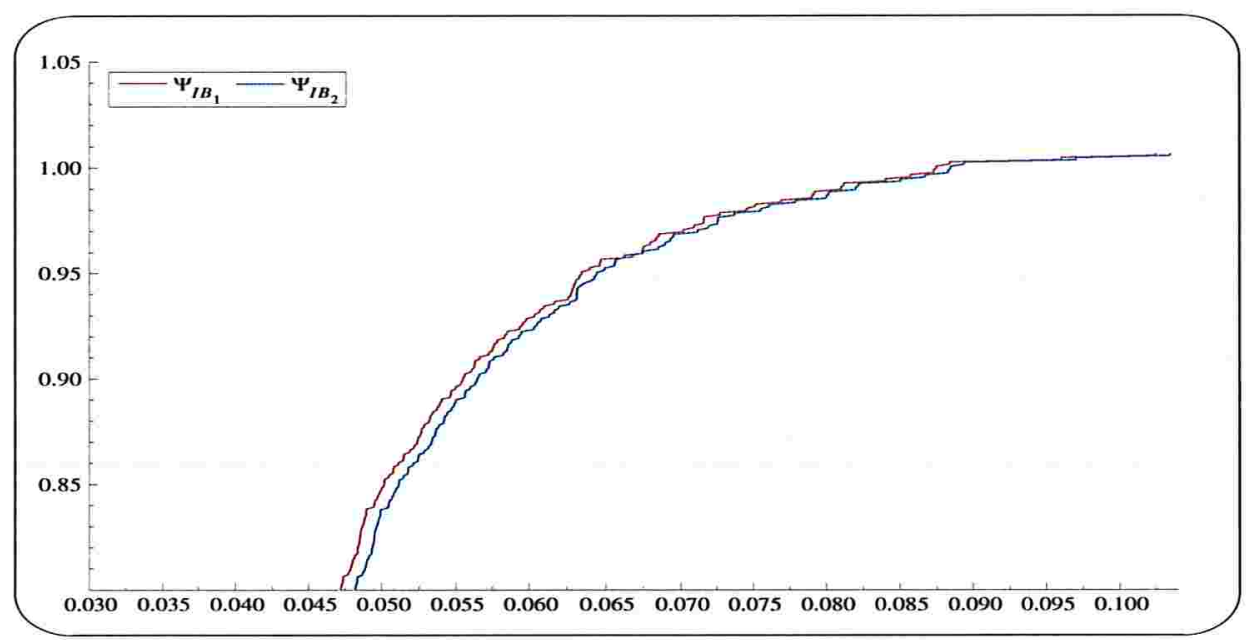

Figura 4.3: Distribuição empírica dos máximos para os modelos $\Psi_{I B_{1}}$ e $\Psi_{I B_{2}}$ para $\mathrm{n}=1000$. 


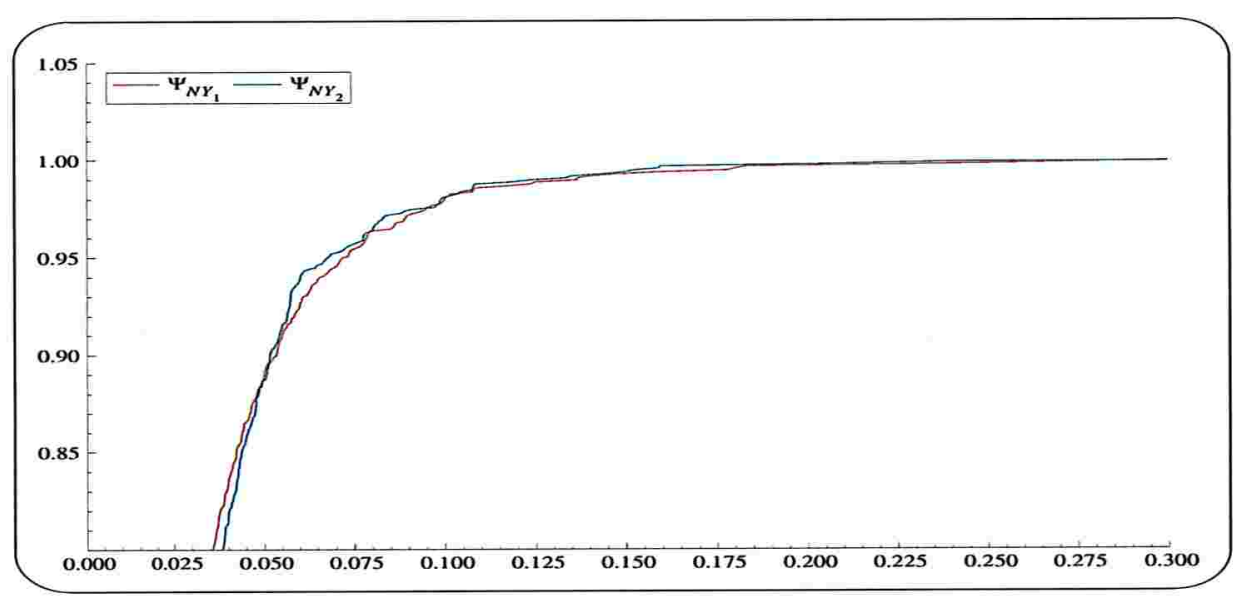

Figura 4.4: Distribuição empírica dos máximos para os modelos $\Psi_{N Y_{1}}$ e $\Psi_{N Y_{2}}$ para $\mathrm{n}=1000$.

A Tabela 4.2 apresenta os valores críticos ao n.s. $5 \%$ para os quatro modelos. Os valores dados na primeira coluna foram estimados utilizando valores de parâmetros verdadeiros. Por exemplo, para os modelo $\Psi_{I B_{1}}$ foi considerado como estimado $\Psi_{I B_{2}}$ e vice-versa. O mesmo ocorre com os valores de $\Psi_{N Y_{i}}, i=1,2$. Os valores da segunda coluna foram estimados utilizando valores próximos considerados como provenientes de estimação. Os valores entre parênteses são os desvios associados com as estimativas pelos percentis, considerando os percentis amostrais com distribuição assintótica normal.

\begin{tabular}{c|cc}
\hline \multirow{2}{*}{ Modelos } & \multicolumn{2}{|c}{ Valores Críticos } \\
& Verdadeiros & Estimados \\
\hline$\Psi_{I B_{1}}$ & 0.063765 & 0.064462 \\
& $(0.00127)$ & $(\mathbf{0 . 0 0 1 4 8 )}$ \\
$\Psi_{I B_{2}}$ & 0.064462 & 0.063765 \\
& $(0.00148)$ & $(0.00127)$ \\
$\Psi_{N Y_{1}}$ & 0.072028 & 0.070631 \\
& $(0.00174)$ & $(\mathbf{0 . 0 0 1 3 7 )}$ \\
$\Psi_{N Y_{2}}$ & 0.076631 & 0.072028 \\
& $(0.00137)$ & $(0.00174)$ \\
\hline \hline
\end{tabular}

Tabela 4.2: Valores críticos para os modelos para $n=1000$ e nível de significância $5 \%$ baseados em 3000 replicações. 
A Tabela 4.3 apresenta o tamanho real do teste caso os valores críticos fossem estimados por simulação utilizando valores de parâmetros diferentes do real.

$\mathrm{Na}$ Tabela 4.3 podemos verificar que o tamanho real é próximo do nominal quando os parâmetros utilizados não estão muito distantes dos valores verdadeiros garantindo a utilização da metodologia para estimar os valores críticos. Observe entretanto, que não é possível utilizar um valor crítico único. Por exemplo, utilizando os valores $\Psi_{I B_{1}}$ para estimar o valor crítico quando $\Psi_{N Y_{1}}$ for o modelo verdadeiro temos tamanhos reais dos testes iguais a $6.5 \%$ e $3.6 \%$ para níveis de significância iguais a $5 \%$ e $1 \%$, respectivamente. Por outro lado, podemos verificar que se utilizarmos os valores críticos considerando $\Psi_{I B_{2}}$ nas séries simuladas como parâmetro verdadeiro quando na verdade o conjunto de parâmetros verdadeiro é o $\Psi_{I B_{1}}$ o tamanho real do teste é de $4.7 \%$ e $0.99 \%$ para níveis $5 \%$ e $1 \%$ respectivamente, e se utilizarmos $\Psi_{I B_{1}}$, no lugar $\Psi_{I B_{2}}$ obtemos tamanhos reais de $5.5 \%$ e $1.01 \%$.

\begin{tabular}{|c|c|c|c|c|c|c|c|c|}
\hline \multirow{3}{*}{$\begin{array}{c}\text { Modelo } \\
\text { Considerado } \\
\text { Verdadeiro }\end{array}$} & \multicolumn{8}{|c|}{ Parâmetro Utilizado na Estimação } \\
\hline & \multicolumn{2}{|c|}{$\hat{\Psi}_{I B_{1}}$} & \multicolumn{2}{|c|}{$\hat{\Psi}_{I B_{2}}$} & \multicolumn{2}{|c|}{$\hat{\Psi}_{N Y_{1}}$} & \multicolumn{2}{|c|}{$\hat{\Psi}_{N Y_{2}}$} \\
\hline & 5 & 1 & 5 & 1 & 5 & 1 & 5 & 1 \\
\hline$\Psi_{I B_{1}}$ & - & - & 4.7 & 0.99 & 2.4 & 0.0 & 3.6 & 0.0 \\
\hline$\Psi_{I B_{2}}$ & 5.5 & 1.01 & - & - & 6.2 & 3.5 & 3.9 & 0.0 \\
\hline$\Psi_{N Y_{1}}$ & 6.5 & 3.6 & 6.2 & 3.5 & - & - & 5.2 & 1.01 \\
\hline$\Psi_{N Y_{2}}$ & 5.5 & 3.4 & 5.5 & 3.2 & 5.2 & 0.99 & - & - \\
\hline
\end{tabular}

Tabela 4.3: Tamanho real do teste em porcentagem, quando o valor crítico é estimado utilizando parâmetros diferentes dos verdadeiros. 


\subsubsection{Distribuição de Referência}

Apresentamos a seguir as estimativas dos percentis para tamanhos de amostra iguais a 1000 e 1500 utilizando os valores dos parâmetros estimados para as duas séries empíricas. A Tabela 4.4 apresenta os percentis para os máximos $S^{*}$. Esses valores podem ser utilizados como um teste geral para verificar se existe pelo menos um valor influente.

\begin{tabular}{lc|cc}
\hline \hline Tamanho & \multirow{2}{*}{ Modelos } & \multicolumn{2}{|c}{ Percentis } \\
Amostral & & $95 \%$ & $99 \%$ \\
\hline \multirow{2}{*}{1000} & $\Psi_{I B_{1}}$ & 0.06376 & 0.12819 \\
& $\Psi_{N Y_{1}}$ & 0.07203 & 0.17130 \\
& & & \\
\multirow{2}{*}{1500} & $\Psi_{I B_{1}}$ & 0.06693 & 0.13981 \\
& $\Psi_{N Y_{1}}$ & 0.08391 & 0.19258 \\
\hline \hline
\end{tabular}

Tabela 4.4: Percentis de $S^{*}$. 


\subsection{Aplicações}

Nesta seção apresentaremos a aplicação do teste proposto às séries simuladas e reais apresentadas no Capítulo 2. Nas séries simuladas consideramos inicialmente séries sem a inclusão de outliers e mudanças estruturais. Depois serão incorporados outliers e mudanças estruturais, embora, como comentado inicialmente, não exista uma relação um a um entre valores influentes e outliers e mudanças estruturais. Finalmente, o teste será aplicado às duas séries reais em estudo na tese.

\subsubsection{Séries Simuladas}

Essa seção apresentará os resultados deste método aplicado a séries simuladas que foram propostas no Capítulo 2. A metodologia será inicialmente aplicada às séries sem a presença de outliers e mudanças estruturais. Posteriormente, as séries com a presença de valores atípicos serão analisadas.

\subsubsection{Série Sem Outliers e Mudanças Estruturais}

Nesta subseção serão apresentados os resultados das simulações realizadas, a fim de estudarmos o comportamento da estatística apresentada quando aplicada ao modelo de volatilidade estocástica.

A Figura 4.5 ilustra o método aplicado as séries simuladas utilizando os conjuntos de parâmetros $\Psi_{1}\left(0.98 ; 0.12^{2}\right)$ e $\Psi_{2}\left(0.95 ; 0.20^{2}\right)$, ou seja, não foi inserido nenhum tipo de outlier. Como para estes conjuntos de parâmetros as estimativas foram próximas dos valores reais $\left(\hat{\Psi}_{I B_{1}}=\left(0.9836 ; 0.1268^{2}\right)\right.$ e $\left.\hat{\Psi}_{N Y_{1}}=\left(0.9231 ; 0.1910^{2}\right)\right)$ podemos utilizar os valores críticos da Tabela 4.4. Na parte superior da figura podemos verificar a série simulada e na parte inferior podemos avaliar o comportamento da estatística proposta para a deteç̧ão de valores influentes em modelos de volatilidade. 


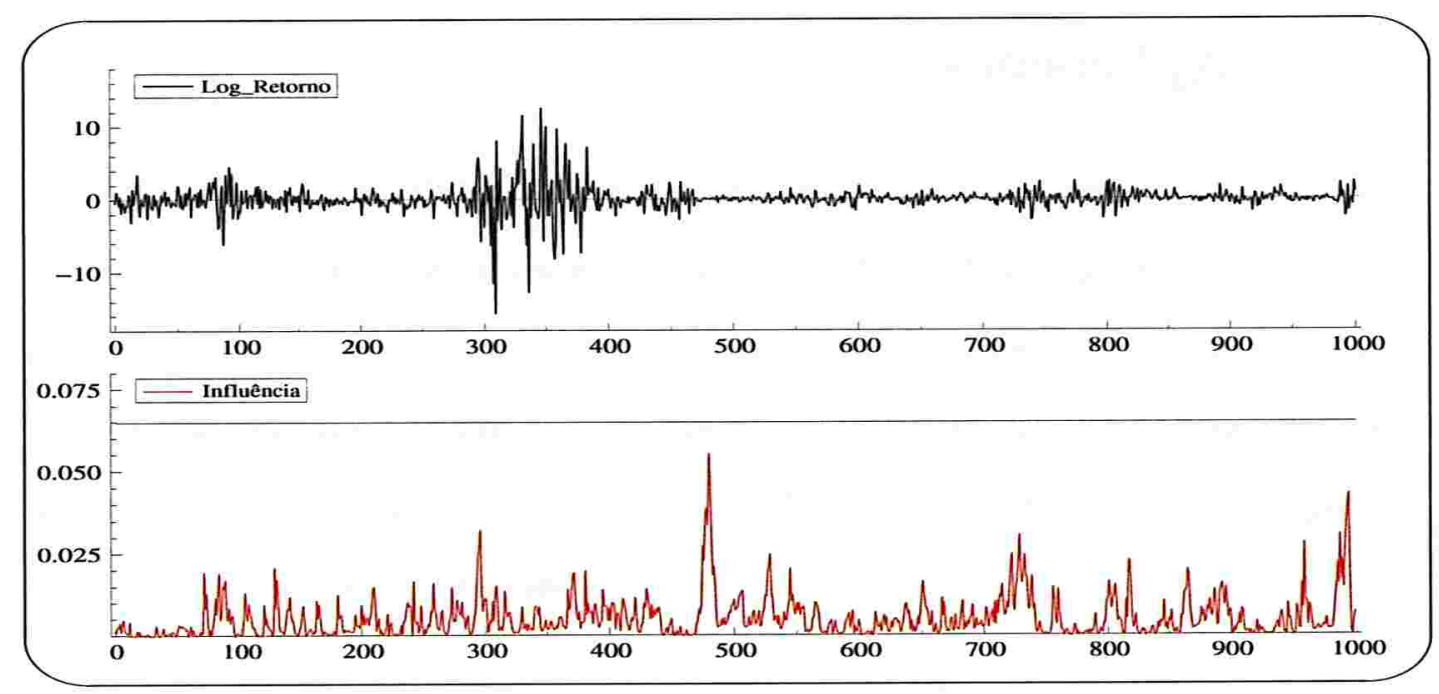

Figura 4.5: Série exemplo para conjunto de parâmetro $\Psi_{1}$ com valor crítico ao n.s. $5 \%$ igual a 0.0647 .

A Figura 4.6 ilustra o método aplicado à série simulada utilizando o conjunto de parâmetros $\Psi_{2}$, ou seja, não foi inserido nenhum tipo de outlier e os parâmetros para o modelo de volatilidade foram 0.95 e 0.2 para os parâmetros de persistência e $\sigma_{\eta}$, respectivamente. Os valores estimados estão próximos de $\Psi_{N Y_{1}}$. A partir da Figura 4.6 verificamos que nenhuma observação foi detectada como influente, pois $S^{*}$ é menor do que o valor crítico 0.072 para nível de significância 5\% (ver Tabela 4.4 $\left.\operatorname{para} \Psi_{N Y_{1}}\right)$. 


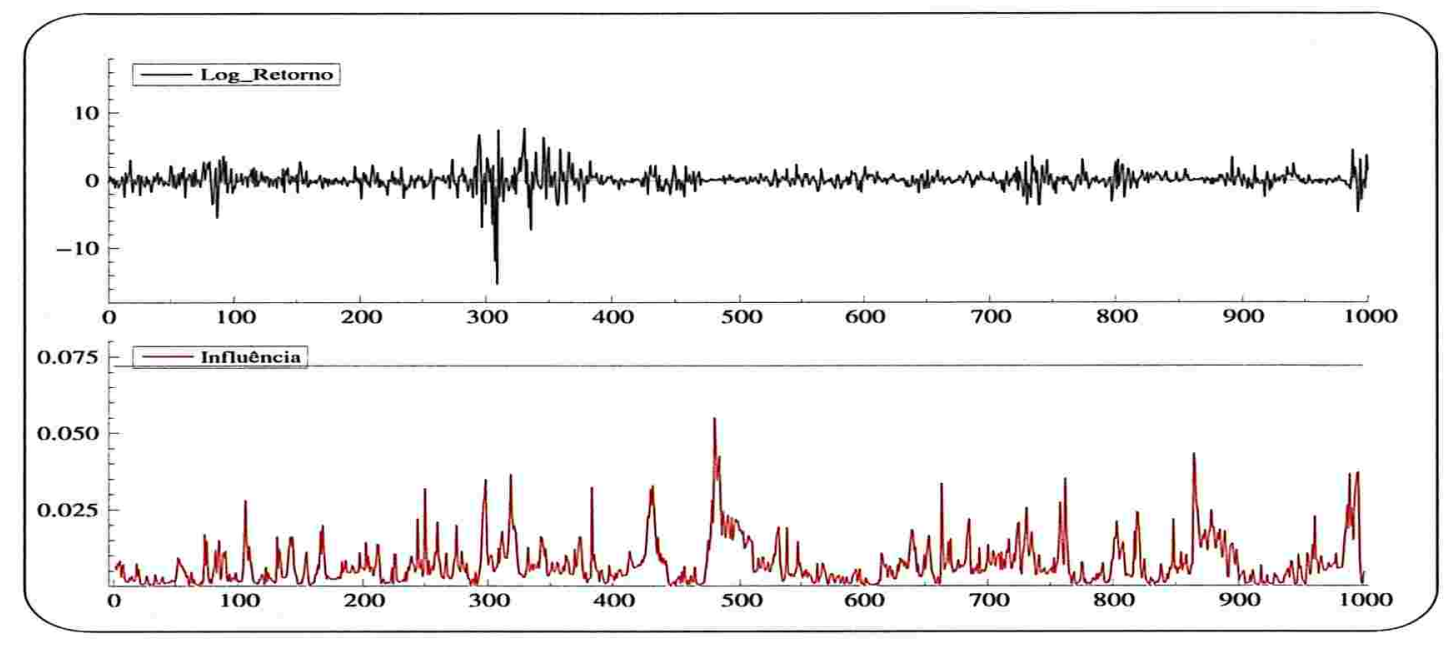

Figura 4.6: Série exemplo para conjunto de parâmetro $\Psi_{2}$ com valor crítico ao n.s. $5 \%$ igual a 0.072 .

\subsubsection{Série Com Outlier e Mudanças Estruturais}

Agora, devemos verificar o comportamento da estatística quando tratamos de séries com valores espúrios. Nesse caso foram simuladas séries de tamanhos e conjuntos de parâmetros como descritos anteriormente. Os resultados foram análogos para os modelos $\Psi_{3}$ e $\Psi_{4}$, por isso, nos restringimos a apresentar os resultados apenas para a série simulada para o modelo $\Psi_{3}$.

A Figura 4.7 apresenta a série com todas as configurações de valores aberrantes consideradas e as estimativas das estatísticas. Pode-se notar que a estatística apresentou aumento significante do seu valor onde os valores espúrios foram inseridos e a estatística conseguiu distinguir os valores inseridos.

Vale ressaltar que a estatística obteve um aumento muito maior quando consideramos a existência de valores aberrantes em blocos ou quando tratamos o caso de 


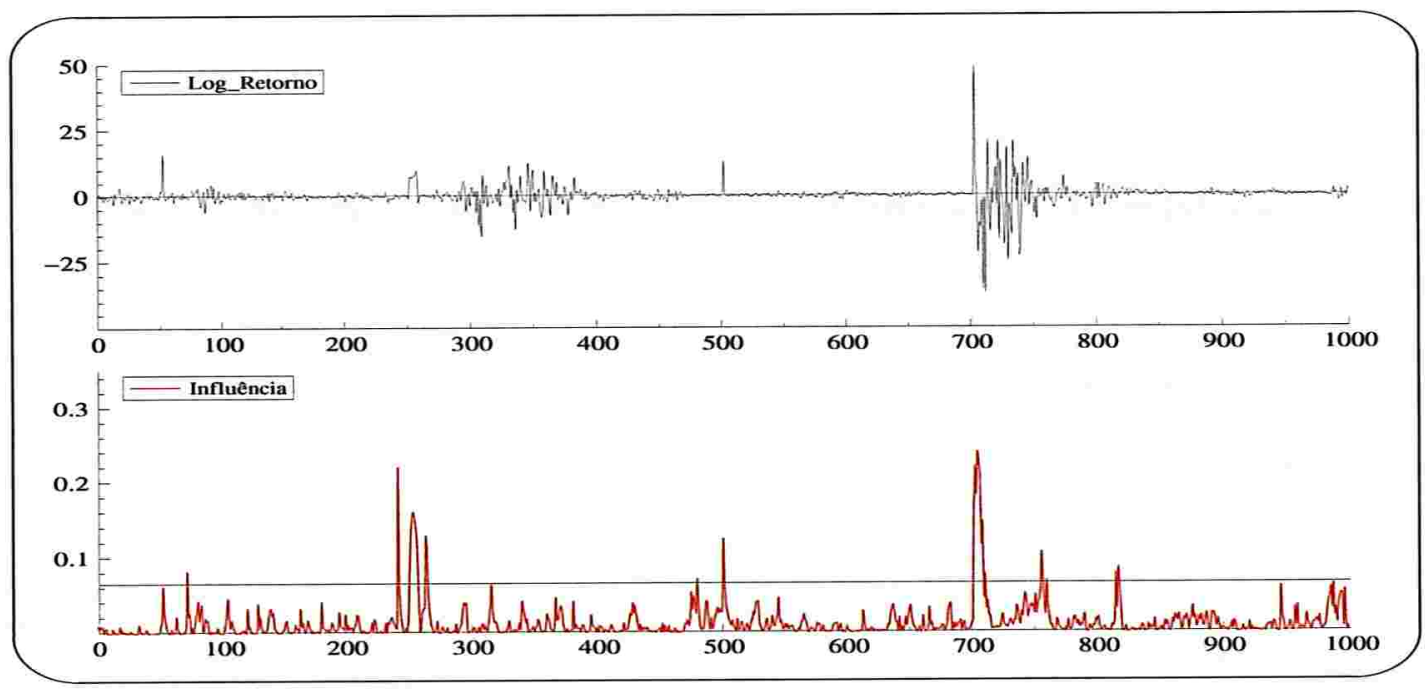

Figura 4.7: Série exemplo para conjunto de parâmetro $\Psi_{3}$ com valor crítico de $5 \%$.

um outlier de inovação. Por outro lado, apesar do valor da estatística ter sido ligeiramente menor, quando tratamos de um valor espúrio isolado, tais valores também são identificados pela estatística de forma eficiente. A Figura 4.7 ilustra que o teste detectou corretamente todos os outliers inseridos no processo gerador de dados. Por outro lado verificamos que um total de 9 observações foram detectadas incorretamente e outras 4 observações foram detectadas imediatamente após o IO que foi inserido. 


\subsubsection{Análise da Série do Ibovespa}

Nesta subseção analisaremos a série de retornos do índice da bolsa do Estado de São Paulo apresentada no Capítulo 2. Utilizaremos o método proposto para caracterizarmos os valores influentes que podem ser encontrados na série.

A Figura 4.8 ilustra a série de retornos, a estatística proposta e os valores caracterizados como influentes.

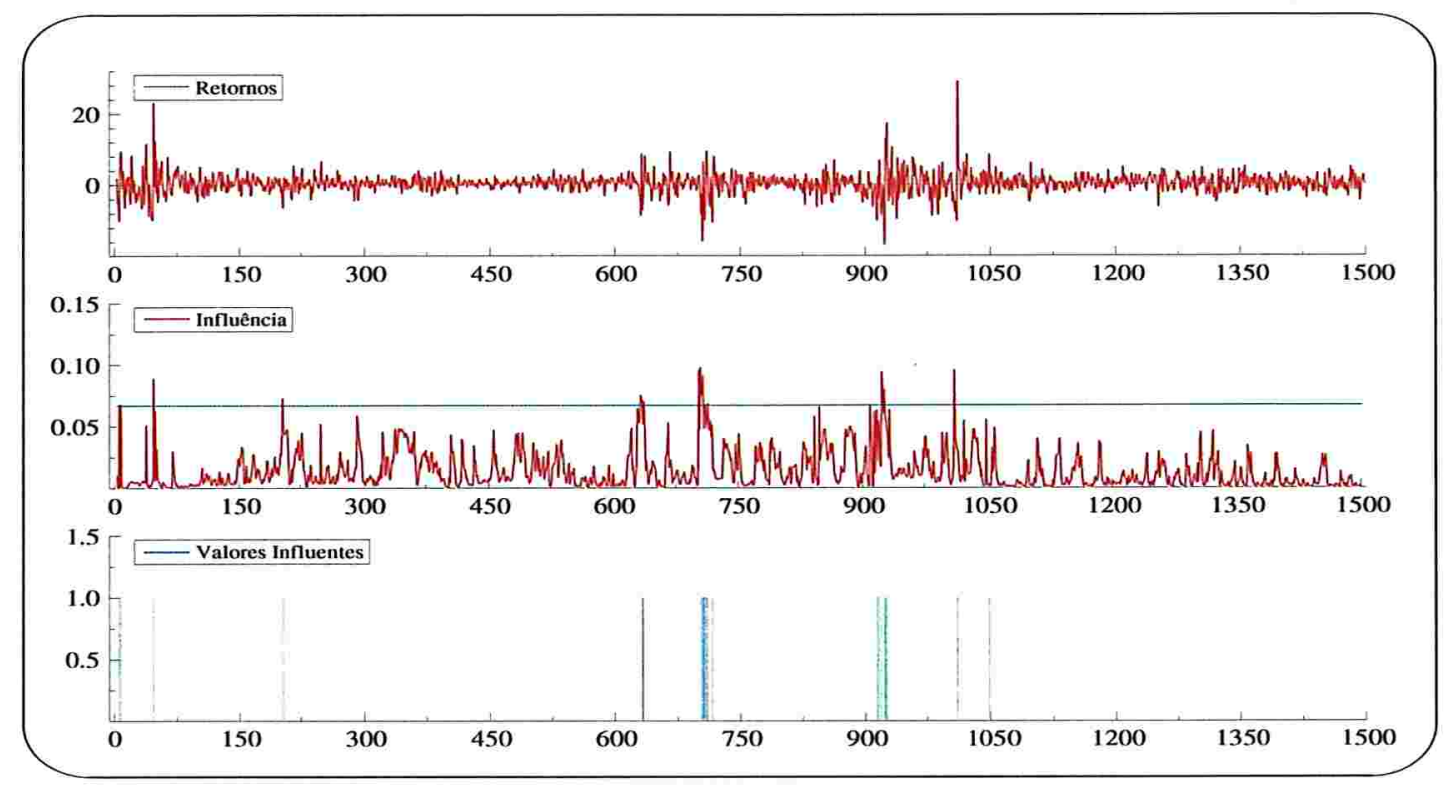

Figura 4.8: Gráfico para análise de influência do Ibovespa: a)Retornos. b) Estatística do teste com valor de corte para n.s. $5 \%$ da Tabela 4.2. c) Observações detectadas como influentes. 
A estimativas dos parâmetros para o modelo foram 0.9811 e 0.2047 para o parâmetro de persistência $\phi$ e a variância $\sigma_{\eta}$ e os desvios padrões associados de 0.0112 e 0.0553 respectivamente.

As Figuras 4.9 e 4.10 apresentam de forma mais clara em que data (posição) os valores detectados como influentes se encontram. Nota-se que valores influentes foram caracterizados ocorrendo isoladamente e em blocos como apresentado claramente em $t=700$.

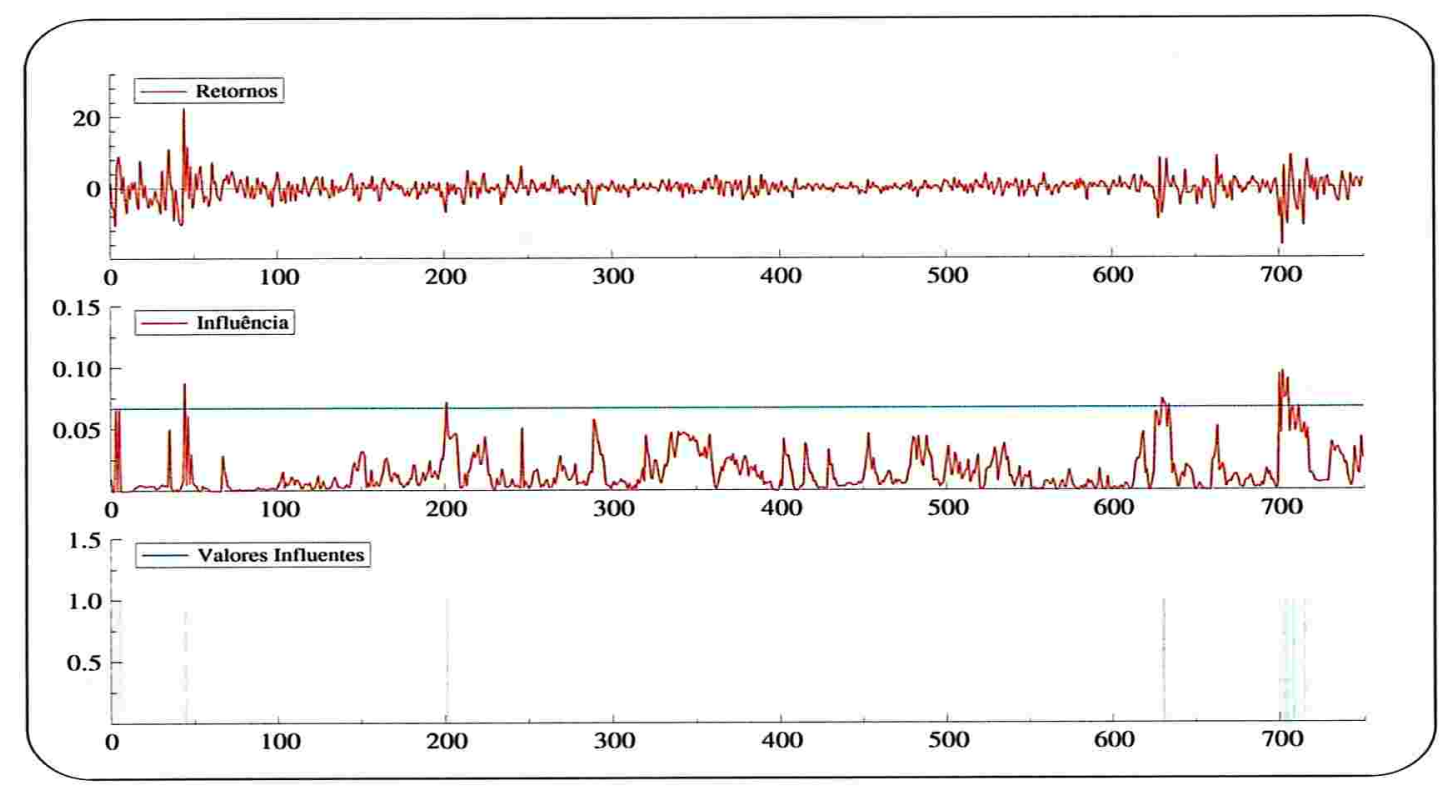

Figura 4.9: Gráfico das 750 observações iniciais para análise de influência do Ibovespa: a)Retornos. b) Estatística do teste com valor de corte para n.s. 5\% da Tabela 4.2. c) Observações detectadas como influentes. 


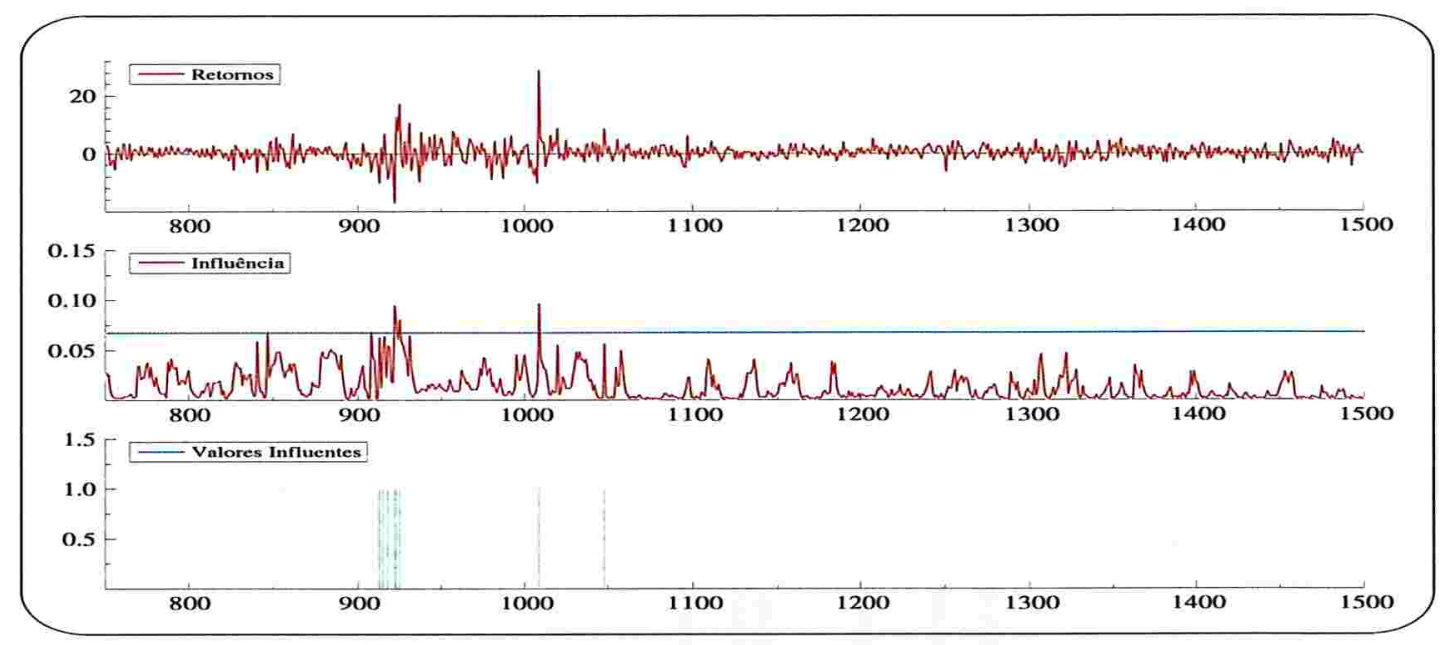

Figura 4.10: Gráfico das 750 observações finais dos retornos e influência do Ibovespa: a)Retornos. b) Estatística do teste com valor de corte para n.s. 5\% da Tabela 4.2. c) Observações detectadas como influentes.

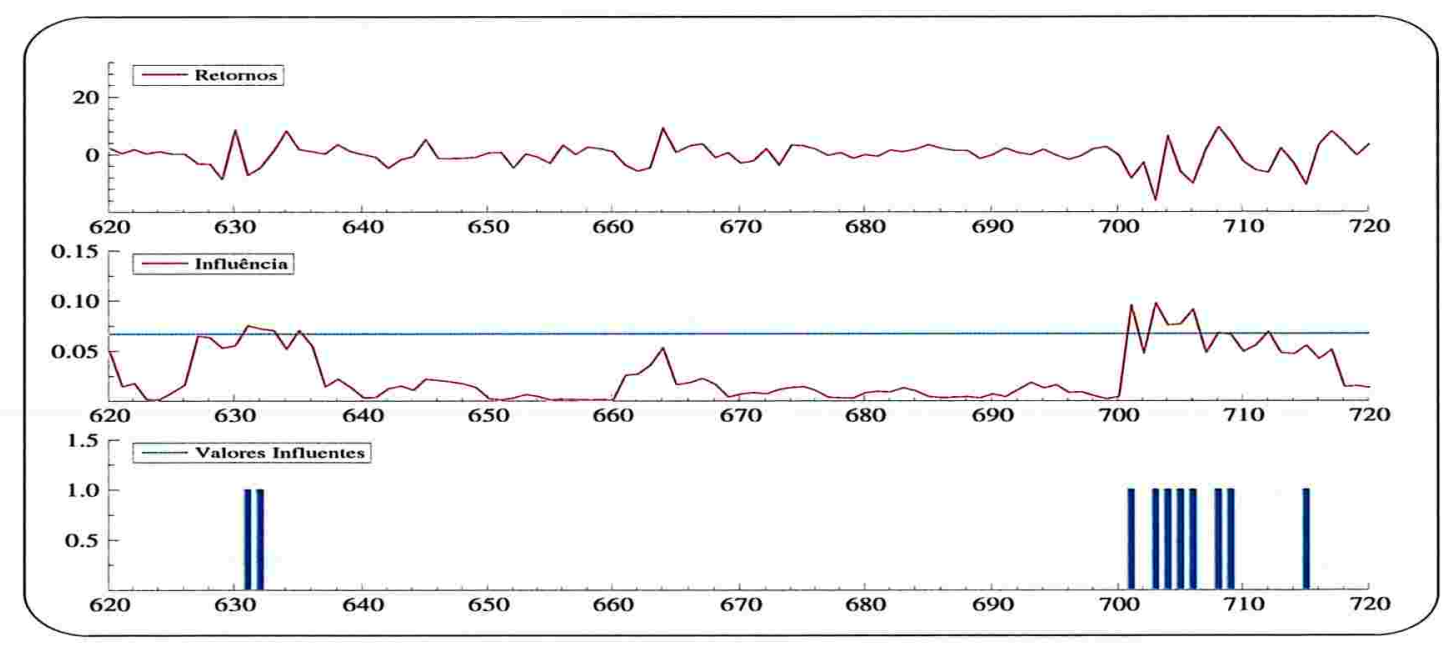

Figura 4.11: Gráfico das observações entre a posição 620 e 720 para análise de influência do Ibovespa: a)Retornos. b) Estatística do teste com valor de corte para n.s. $5 \%$ da Tabela 4.2. c) Observações como influentes. 


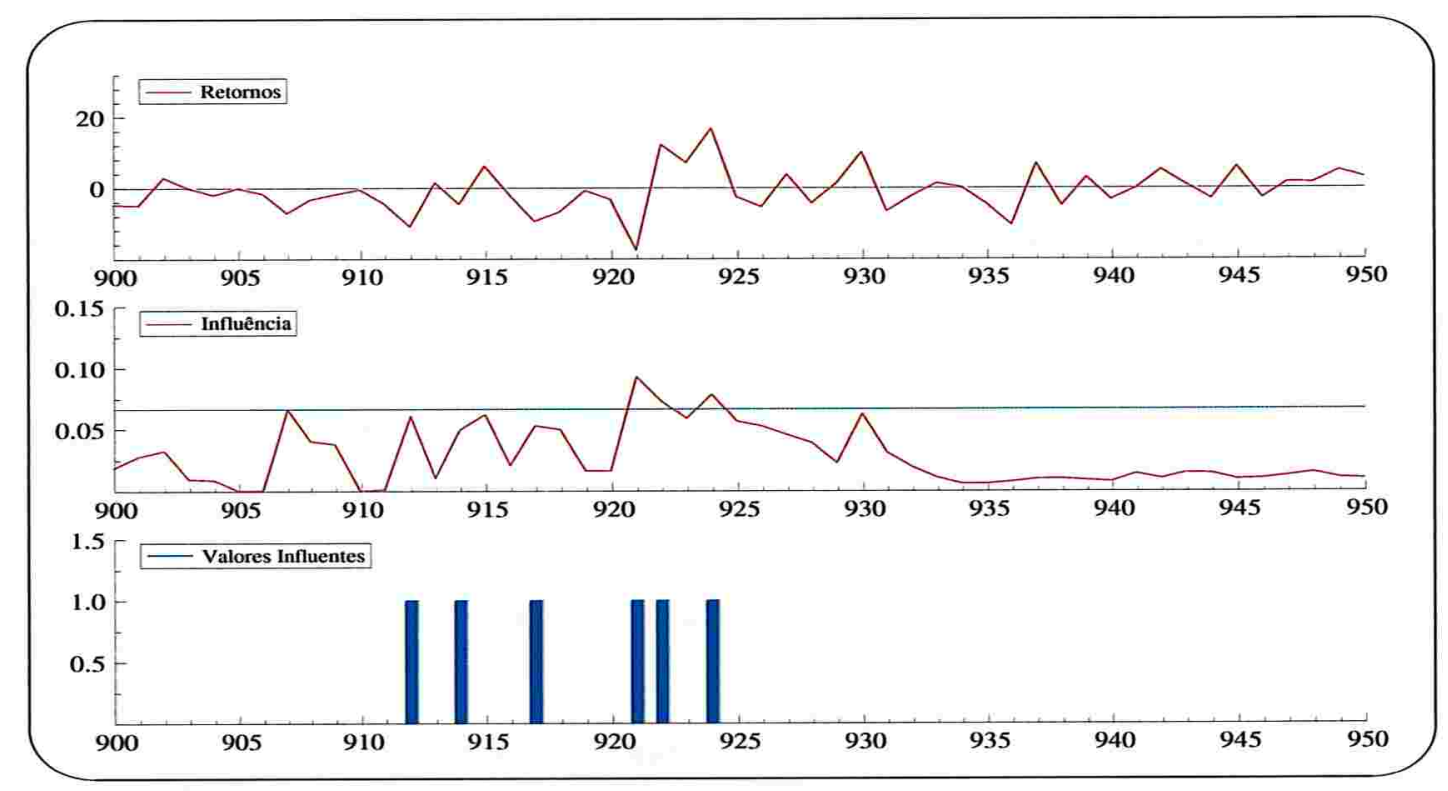

Figura 4.12: Gráfico das observações entre a posição 900 e 950 para análise de influência do Ibovespa: a)Retornos. b) Estatística do teste com valor de corte para n.s. $5 \%$ da Tabela 4.2. c) Observações detectadas como influentes.

A Tabela 4.5 apresenta os valores da série de retornos do Ibovespa que foram caracterizados como influentes. Na tabela podemos verificar a data de ocorrência assim como a localização na ilustração dada nas figuras anteriormente. O valor da estatística proposta encontra-se na última coluna, onde podemos verificar o valor assumido pela estatística na data da ocorrência do valor influente.

Pode-se notar que o método além de detectar valores influentes que ocorreram isoladamente, ele detectou a presença de um bloco de valores influentes datado de 27 a 30 de outubro de 1997, época da segunda crise asiática 


\begin{tabular}{ccrc}
\hline \hline Data & Localização & Retorno & Estatística \\
\hline $10 /$ jan/1995 & 4 & $-10.47 \%$ & 0.0648 \\
$12 /$ jan/1995 & 6 & $9.22 \%$ & 0.0727 \\
$10 /$ mar/1995 & 45 & $22.72 \%$ & 0.0887 \\
$26 /$ out/1995 & 202 & $-6.84 \%$ & 0.0723 \\
$17 /$ jul/1997 & 631 & $-7.58 \%$ & 0.0743 \\
$18 /$ jul/1997 & 632 & $-4.87 \%$ & 0.0724 \\
$23 /$ out/1997 & 701 & $-8.59 \%$ & 0.0951 \\
$27 /$ out/1997 & 703 & $-16.31 \%$ & 0.0972 \\
$28 /$ out/1997 & 704 & $6.14 \%$ & 0.0750 \\
$29 /$ out/1997 & 705 & $-6.30 \%$ & 0.0761 \\
$30 /$ out/1997 & 706 & $-10.42 \%$ & 0.0908 \\
$03 /$ nov/1997 & 708 & $9.17 \%$ & 0.0675 \\
$04 /$ nov/1997 & 709 & $3.85 \%$ & 0.0654 \\
$12 /$ nov/1997 & 715 & $-10.84 \%$ & 0.0655 \\
$18 /$ ago/1998 & 907 & $-6.75 \%$ & 0.0671 \\
$25 /$ ago/1998 & 912 & $-10.57 \%$ & 0.0812 \\
$27 /$ ago/1998 & 914 & $-4.24 \%$ & 0.0702 \\
03/set/1998 & 917 & $-9.10 \%$ & 0.0736 \\
04/set/1998 & 918 & $-6.41 \%$ & 0.0650 \\
$10 /$ set/1998 & 921 & $-17.32 \%$ & 0.0937 \\
$11 /$ set/1998 & 922 & $12.49 \%$ & 0.0738 \\
$15 /$ set/1998 & 924 & $17.04 \%$ & 0.0793 \\
$15 /$ jan/1999 & 1008 & $28.73 \%$ & 0.0954 \\
$15 /$ mar/1999 & 1047 & $8.31 \%$ & 0.0655 \\
\hline \hline
\end{tabular}

Tabela 4.5: Valores influentes identificados no Ibovespa: Data de ocorrência, posição, retorno e valor da estatística. Valor crítico ao n.s. $5 \%$ igual a 0.064 .

Podemos verificar pela Tabela 4.5 que o método detectou 24 observações como influentes. Das observações caracterizadas como influentes, 17 estão relacionadas à crises que impactaram diretamente o mercado financeiro.

Em 10 de março tivemos a introdução de intrabandas cambiais, no início da segunda quinzena do mês de julho de 97 a primeira crise da Ásia afetou o mercado financeiro mundial. No fim de outubro de 1997 a segunda crise da Ásia provocou grandes quedas no índice bovespa, assim como em todo o mercado mundial. No ano seguinte, a moratória da Rússia no fim de agosto e a forte queda das bolsas em setembro também foram detectados pelo procedimento proposto neste capítulo. 


\subsubsection{Análise da Série da NYSE}

Nesta subseção analisaremos a série de retornos da NYSE apresentada no Capítulo

2. Utilizaremos o método proposto para caracterizarmos os valores influentes que podem ser encontrados na série.

A Figura 4.13 ilustra a série de retornos, a estatística proposta e os valores caracterizados como influentes.

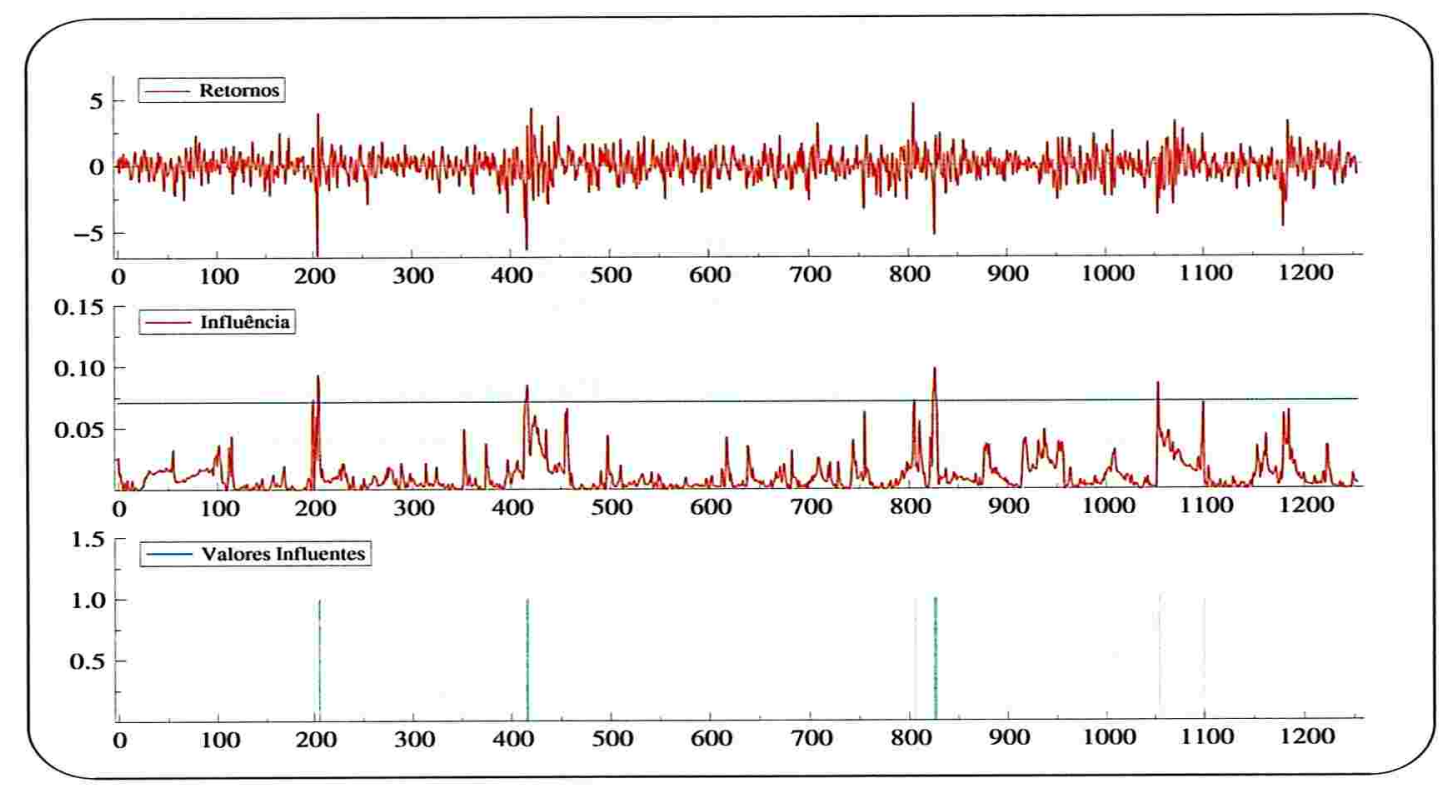

Figura 4.13: Gráfico para análise de influência do NYSE: a)Retornos. b) Estatística do teste com valor de corte para n.s. $5 \%$ da Tabela 4.2. c) Observações detectadas como influentes. 
A estimativas dos parâmetros para o modelo foram 0.9310 e 0.1813 para o parâmetro de persistência $\phi$ e a variância $\sigma_{\eta}$ e os desvios associados de 0.00211 e 0.00516 respectivamente.

As Figuras 4.14 e 4.15 apresentam de forma mais clara em que data (posição) os valores detectados como influentes se encontram.

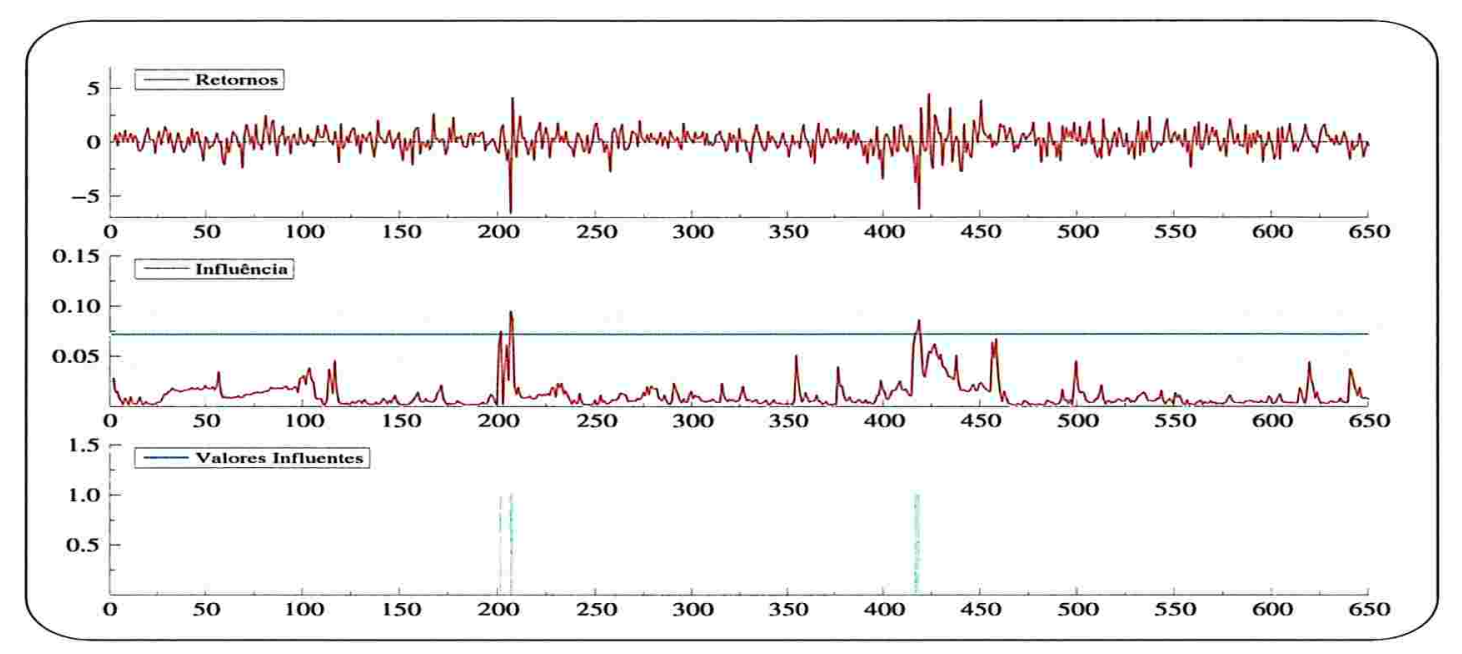

Figura 4.14: Gráfico das 650 observações iniciais para Análise de Influência do NYSE: a)Retornos. b) Estatística do teste com valor de corte para n.s. 5\% interpolado dos valores da Tabela 4.2. c) Observações detectadas como influentes. 


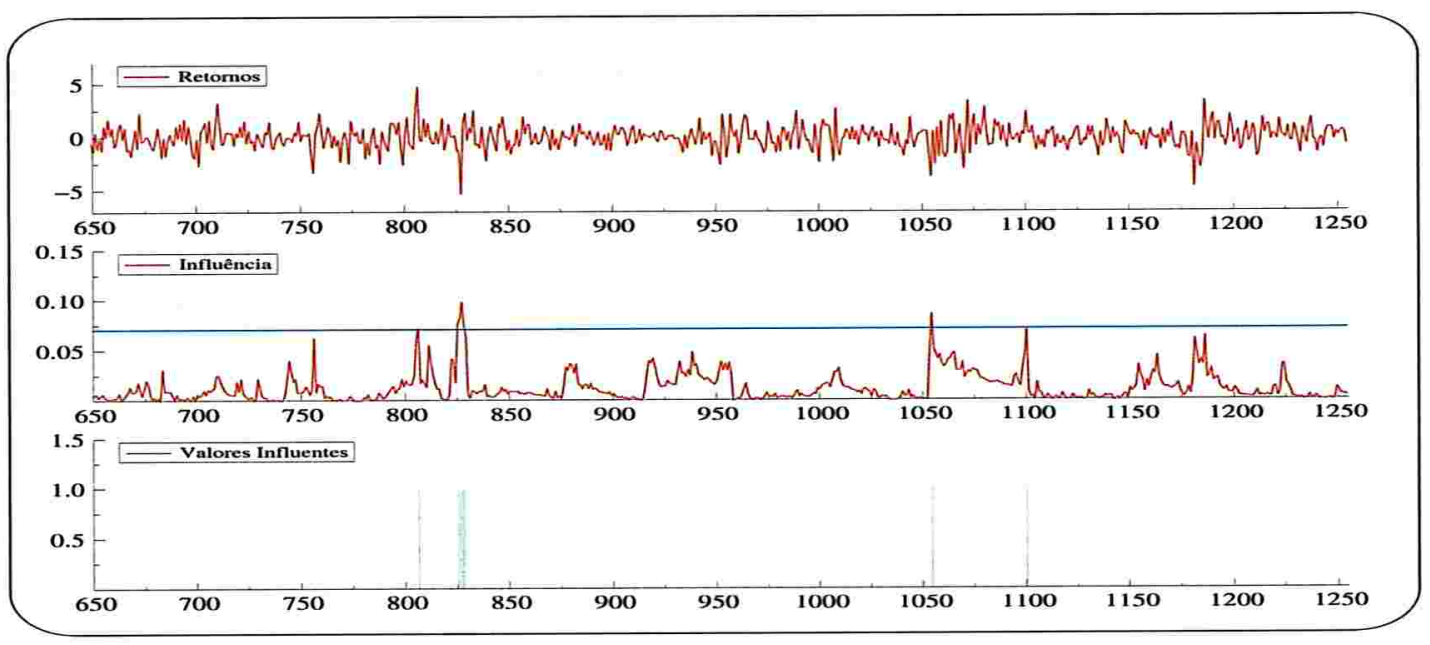

Figura 4.15: Gráfico das 605 observações finais para análise de influência do NYSE: a)Retornos. b) Estatística do teste com valor de corte para n.s. 5\% interpolado dos valores da Tabela 4.2. c) Observações detectadas como influentes.

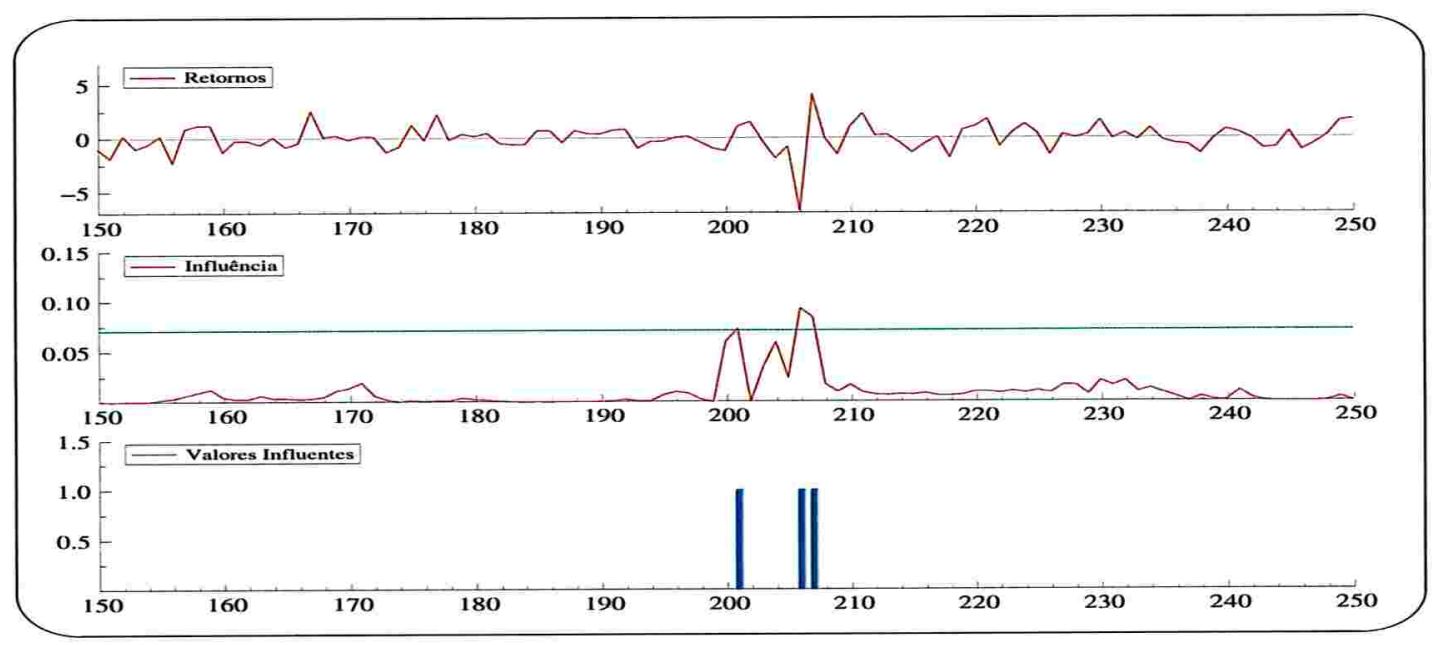

Figura 4.16: Gráfico das observações entre a posição 150 e 250 para análise de influência do NYSE: a)Retornos. b) Estatística do teste com valor de corte para n.s. $5 \%$ interpolado dos valores da Tabela 4.2. c) Observações detectadas como influentes. 


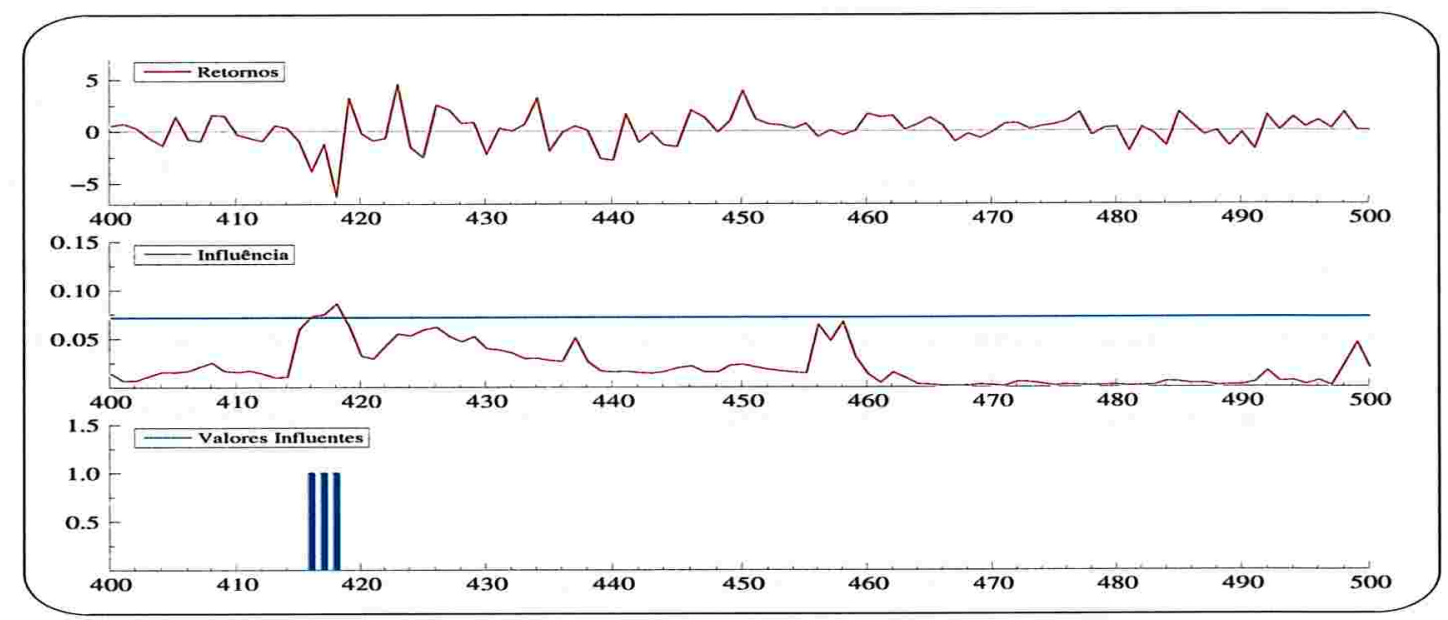

Figura 4.17: Gráfico das observações entre a posição 400 e 500 para análise de influência do NYSE: a)Retornos. b) Estatística do teste com valor de corte para n.s. $5 \%$ interpolado dos valores da Tabela 4.2. c) Observações detectadas como influentes.

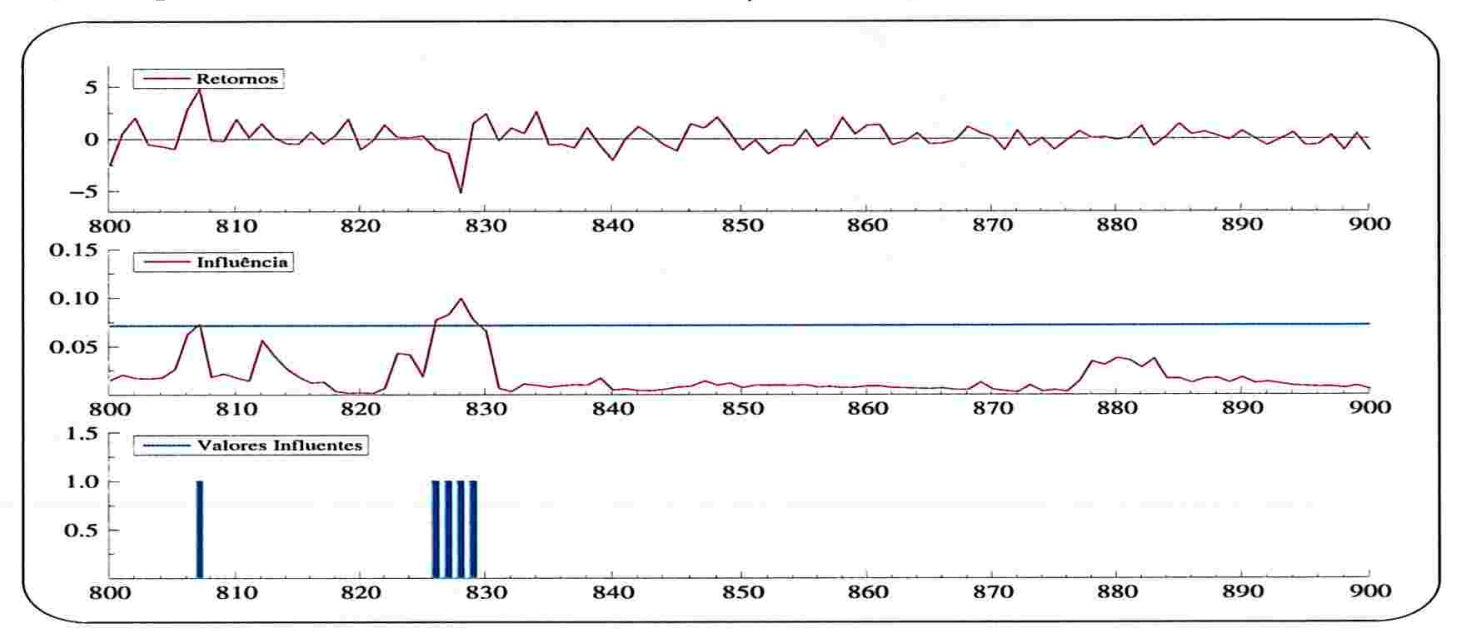

Figura 4.18: Gráfico das observações entre a posição 800 e 900 para análise de influência do NYSE: a)Retornos. b) Estatística do teste com valor de corte para n.s. $5 \%$ interpolado dos valores da Tabela 4.2. c) Observações detectadas como influentes.

Nas Figuras 4.16, 4.17 e 4.18 é possível distinguir os blocos de valores influentes. Verificamos que o procedimento detectou um total de 3 blocos de valores influentes, um bloco de tamanho 2 , um bloco de tamanho 3 e um bloco de tamanho 5 . 
A Tabela 4.6 apresenta os valores da série de retornos da NYSE que foram caracterizados como influentes. Na tabela podemos verificar a data de ocorrência assim como a localização na ilustração dada nas figuras anteriormente. O valor da estatística proposta encontra-se na última coluna, onde podemos verificar o valor assumido pela estatística na data da ocorrência do valor influente. Podemos verificar que alguns blocos de valores influentes foram caracterizados e coincidem com alguns valores obtidos pelo procedimento proposto no Capítulo 3 .

\begin{tabular}{ccrc}
\hline \hline Data & Localização & Retorno & Estatística \\
\hline $20 / 10 / 1997$ & 201 & $1.139 \%$ & 0.0738 \\
$27 / 10 / 1997$ & 206 & $-6.791 \%$ & 0.0940 \\
$28 / 10 / 1997$ & 207 & $4.113 \%$ & 0.0852 \\
$27 / 08 / 1998$ & 415 & $-3.920 \%$ & 0.0721 \\
$28 / 08 / 1998$ & 416 & $-1.300 \%$ & 0.0740 \\
$01 / 09 / 1998$ & 418 & $-6.352 \%$ & 0.0851 \\
$16 / 03 / 2000$ & 807 & $4.748 \%$ & 0.0724 \\
$12 / 04 / 2000$ & 826 & $-1.056 \%$ & 0.0761 \\
$13 / 04 / 2000$ & 827 & $-1.484 \%$ & 0.0821 \\
$14 / 04 / 2000$ & 828 & $-5.275 \%$ & 0.0749 \\
$15 / 04 / 2000$ & 829 & $1.415 \%$ & 0.0762 \\
$12 / 03 / 2001$ & 1055 & $-3.729 \%$ & 0.0865 \\
$16 / 05 / 2001$ & 1101 & $2.372 \%$ & 0.0703 \\
$14 / 09 / 2001$ & 1182 & $-4.701 \%$ & 0.0771 \\
\hline \hline
\end{tabular}

Tabela 4.6: Valores ifluentes identificados na NYSE.

Pode-se notar que a metodologia apresentada neste capítulo, detetou menos observações influentes do que o método bayesiano no caso da série de retornos da NYSE. O procedimento detectou um total de 14 observações influentes, dessas 11 estão relacionadas às crises do mercado financeiro, dentre as quais podemos citar Ásia em 1997, moratória da Rússia em 1998 e Nasdaq em 2000. 


\subsection{Conclusões}

Neste capítulo apresentamos uma estatística para a determinação da influência das observações. A estatística é baseada em estudos de análise de influência para modelos de regressão proposta em Peña (2005). Visto que estamos modelando uma série temporal consideramos algumas modificações na metodologia inicialmente proposta. Verificamos que o método proposto foi eficaz na caracterização de valores outliers quando ocorrem isolados e em blocos. Foi visto que a estatística também ressalta os valores influentes além daqueles que são caracterizados como outlier. Por exemplo, valores muito pequenos que provocam perturbações para a modelagem da série. 



\section{CAPÍTULO 5}

\section{Influência Local em Modelos de Volatilidade Estocástica}

Neste capítulo serão apresentadas algumas abordagens utilizadas na análise de influência local em modelos de volatilidade estocástica. A partir do artigo de Cook (1986), muitos pesquisadores voltaram suas atenções à análise de influência utilizando o conceito de curvatura normal. Na seção 5.1.1 são apresentadas as medidas propostas por Cook (1986) e Billor e Loynes (1993). Descrevemos na seção 5.1 .2 o conceito de máxima inclinação e máxima curvatura para deteç̧ão de valores influentes. A seção 5.3 estende esse conceito para o modelo de volatilidade estocástica, onde será abordada a análise de valores influentes sob diferentes tipos de perturbações. $\mathrm{Na}$ seção 5.4 mostramos os resultados empíricos que levam a obtenção de pontos críticos para deteç̧ão de valores influentes e, finalmente, o método é aplicado na seção 5.4 às séries do Ibovespa e NYSE. 


\subsection{Influência Local}

Muitos trabalhos para obter influência de perturbações em um modelo seguem a abordagem de exclusão de observações uma a uma. O problema dessa metodologia é que podemos ter subconjuntos de observações influentes conjuntamente. Embora subconjuntos de observações influentes ou múltiplos outliers possam ser identificados através da generalização do método de exclusão, o grande número desses subconjuntos torna o método impraticável.

Cook (1986) introduziu a idéia de influência local como ferramenta para verificar o efeito de pequenas perturbações nos dados. A influência local é baseada na perturbação de um ponto e não na exclusão deste, e através da comparação diferencial das estimativas dos parâmetros antes e depois da perturbação. Cook (1986) propôs a aproximação de influência local da perturbação na superfície da verossimilhança deslocada e verificou através do conceito de curvatura, até então objeto de estudo em geometria diferencial, alguns resultados utilizando modelos de regressão.

Desde então esse método foi amplamente utilizado na análise de influência em diversos modelos estatísticos. Thomas e Cook (1990) aplicaram o método para as predições nos modelos lineares generalizados. Paula (1996) faz uma extensão desse estudo para os modelos de dispersão. Lee e Zhao (1996) utilizam o método em modelos lineares generalizados quando as covariáveis são medidas com erros. No modelo linear misto, Lesafre e Verbeke (1998) também utilizaram o método de influência local e Laurent e Cook (1993) aplicam a técnica em um modelo de regressão não-linear.

Kim (1995) generaliza o método de influência local para modelos de regressão multivariada, considerando somente perturbação de ponderação de casos para todos os parâmetros de interesse. Fung e Tang (1997) consideram esquemas de perturbação 
nas variáveis explicativas e nas variáveis respostas. Kim (1996) estuda influência de casos sobre o estimador de máxima verossimilhança dos parâmetros de transformação em um modelo de regressão multivariado, Jiancheng e Xizhi (1997) usa o método sobre no modelo de riscos proporcionais de Cox e Galea et al.(1997) aplicam o método no modelo de regressão linear elíptico.

Na literatura também podemos encontrar a análise de influência sob a abordagem bayesiana, onde podemos citar o trabalho de Pan et al.(1996) que analisam influência para diagnosticar a adequação do modelo de curva de crescimento com estrutura de covariância simples de Rao.

Billor e Loynes (1993) consideraram uma modificação da função proposta por Cook (1986) de forma a contornar o problema de especificação do modelo e dificuldades para o cálculo de curvatura, como surge da necessidade de obtermos derivadas de segunda ordem. Tal modificação, apresentada na discussão do artigo de Cook (1986), foi inicialmente proposta por Tsai (1986). A partir desta proposta, Billor e Loynes (1993) propuseram a análise de valores influentes utilizando máxima inclinação e direção da função apresentada e dessa forma evitando o cálculo de derivadas de segunda ordem.

Wu e Luo (1993) propuseram a análise da influência global e local através do estudo da superfície de uma variável, tal como o estimador de máxima verossimilhança (EMV), formado pelas perturbações, superfície esta denominada superfície de EMV. O método denominado de aproximação de segunda ordem, é baseado no cálculo das direções correspondente à máxima curvatura local da superfície de EMV.

Wu e Luo (1993) verificaram que, para o caso de espaço paramétrico de dimensão 1, a máxima inclinação da superfície de EMV é a mesma que a direção da máxima 
curvatura da superfície de verossimilhança deslocada de Cook.

Zhang (2004) e Zhang e King (2005) apresentaram uma ferramenta baseada nos artigos de Cook (1986) e Billor e Loynes (1993) para diagnosticar valores influentes em processos GARCH. Neste artigo eles consideraram três tipos de perturbação no modelo de forma a diagnosticar a presença de outlier em uma série financeira. Os autores levaram em consideração para o diagnóstico de valores influentes, o cálculo da inclinação e curvatura a partir dos métodos propostos por Billor e Loynes e Cook, respectivamente.

\subsubsection{Superfície de Influência}

A influência que uma observação exerce sobre a estimativa dos parâmetros pode ser avaliada através da função de verossimilhança. Essa forma de análise foi proposta por Cook e Weisberg (1982) que denominaram tal medida de verossimilhança deslocada. Essa medida foi modificada por Billor e Loynes (1993) a fim de simplificar alguns cálculos na análise e tornar a medida mais robusta. A nova proposta será denominada em nosso trabalho de verossimilhança deslocada modificada.

\subsubsection{Verossimilhança Deslocada}

Considere $L(\Psi)$ a função de log-verossimilhança correspondente ao modelo postulado e associada a um conjunto de observação, $\mathbf{y}$, onde $\Psi$ é o vetor de parâmetros de dimensão $p \times 1$. Através de um vetor $\omega, r \times 1$ tal que $\omega \subset \Omega$ aberto onde $\Omega \in \Re^{r}$ são introduzidas perturbações ao modelo e assim teremos que $L(\Psi \mid \omega)$ denominará a log-verossimilhança correspondente ao modelo quando consideramos inseridas as perturbações. Assuma que exista um ponto $\omega_{0} \in \Omega$ tal que $L\left(\Psi \mid \omega_{0}\right)=L(\Psi)$ para todo $\Psi$, o qual será chamado de vetor de perturbação nula. 
Seja $\hat{\Psi}$ o estimador de máxima verossimilhança (EMV) para $\Psi$, obtido maximizandose $L(\Psi)$, e considere $\hat{\Psi}_{\omega}$ o EMV para $\Psi$ sob $L(\Psi \mid \omega)$. A análise de influência local visa comparar $\hat{\Psi}$ e $\hat{\Psi}_{\omega}$. Estimativas próximas indicam que a perturbação tem pouca influência nas estimativas dos parâmetros do modelo considerado. Por outro lado, distâncias significantes entre as estimativas indicam que o procedimento utilizado para obter as estimativas é sensível a um determinado tipo de perturbação. Cook (1986) propôs uma métrica para a distância entre as estimativas $\hat{\Psi}$ e $\hat{\Psi}_{\omega}$. Essa métrica, conhecida como verossimilhança deslocada, é definida por

$$
L D(\omega)=2\left[L(\hat{\Psi})-L\left(\hat{\Psi}_{\omega}\right)\right]
$$

Desta forma, $L D(\omega)$ será grande se $L(\Psi)$ é fortemente curvada em $\hat{\Psi}$ (indicando que $\Psi$ é estimado com pouca variabilidade). Por outro lado, $L D(\omega)$ será pequena se $L(\Psi)$ é suavemente achatada em $\hat{\Psi}$ (indicando que $\Psi$ é estimado com grande variabilidade). A Figura 5.1 representa o comportamento da medida proposta.

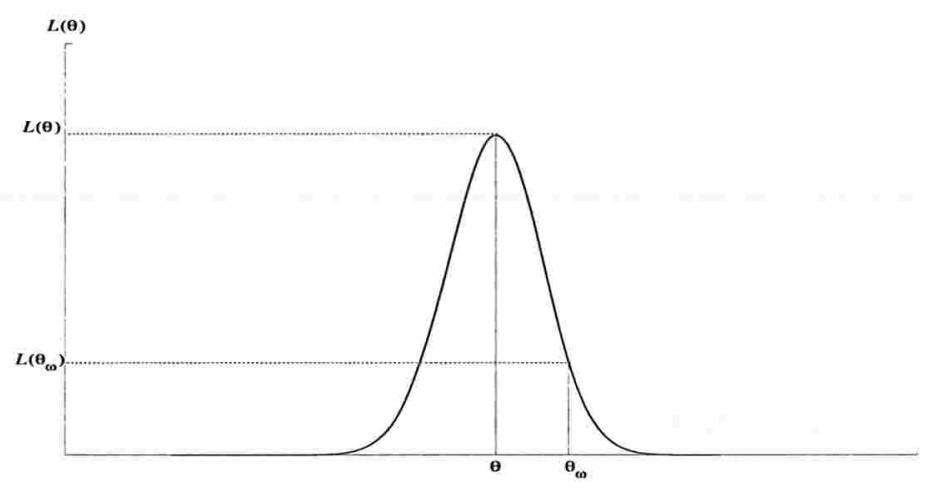

Figura 5.1: Ilustração do desvio de verossimilhança.

Portanto, o gráfico de $L D(\omega)$ versus $\omega$ contém informação a respeito da influência das perturbações. Tal gráfico, denominado por Cook (1986) gráfico de influência é a 
superfície geométrica gerada com $\omega \in \Omega$ por

$$
\alpha(\omega)=\left[\omega_{1}, \cdots, \omega_{n}, L D(\omega)\right]^{\prime}
$$

com $\omega \in \Omega$. Essa superfície em $\Re^{r+1}$ pode ser usada para analisarmos influência de $\omega$ variando em $\Omega$. Como $\hat{\Psi}$ é o EMV de $\Psi$ no modelo não perturbado, segue que $L D(\omega) \geq 0$ para $\omega \in \Omega$. Além disso, como $L D\left(\omega_{0}\right)=0$, podemos concluir que $\omega_{0}$ é um ponto de mínimo local da função $L D(\omega)$. A análise sobre o comportamento de $L D(\omega)$ pode fornecer informações relevantes ao modelo sob investigação. Por exemplo, se os valores de $L D(\omega)$ são próximos, indica que existe estabilidade no modelo ajustado sob a perturbação considerada. Por isso, antes de realizar um estudo de $L D(\omega)$, é importante que o esquema de perturbação esteja bem definido de modo que responda a questões previamente estabelecidas.

A definição de $L D(\omega)$ pode ser interpretada em termos da região de confiança assintótica para $\Psi$

$$
\left\{\Psi \in \Re^{p}: 2[L(\hat{\Psi})-L(\Psi)] \leq \chi_{(1-\alpha, p)}^{2}\right\}
$$

onde $\chi_{(1-\alpha, p)}^{2}$ é o percentil superior de tamanho $1-\alpha$ da distribuição qui-quadrado com $p$ graus de liberdade.

\subsubsection{Verossimilhança Deslocada Modificada}

Billor e Loynes (1993) verificaram que a verossimilhança deslocada tem a propriedade indesejada de depender da parametrização utilizada e fornece resultados não esperados quando não conseguimos escolher versões específicas dos parâmetros relevantes para o problema considerado. Eles apresentaram uma nova métrica análoga à 
proposta por Cook (1986) que é definida por:

$$
L D^{*}(\omega)=-2\left[L(\hat{\Psi})-L\left(\hat{\Psi}_{\omega} \mid \omega\right)\right]
$$

Pode-se notar que com essa nova medida não dependemos da escolha da parametrização, pois $L\left(\hat{\Psi}_{\omega} \mid \omega\right)$ é a verossimilhança maximizada sob o modelo perturbado. A partir dessa nova medida, os estudos para diagnosticar influência podem ser elaborados na mesma linha de Cook para $L D(\omega)$, considerando a superfície geométrica, $\alpha^{*}(\omega)$ dada por

$$
\alpha^{*}(\omega)=\left[\omega_{1}, \cdots, \omega_{n}, L D^{*}(\omega)\right]^{\prime}, \quad \omega \in \Omega
$$

Em nosso trabalho, estaremos utilizando o desvio de verossimilhança modificada de forma a considerarmos a abordagem de curvatura e inclinação para analisar a influência em modelos de volatilidade estocástica, conforme será visto na próxima seção.

\subsubsection{Análise da Superfície de Influência}

Definida a superfície de influência a ser analisada, o próximo passo é apresentar a metodologia que será utilizada para determinarmos se uma determinada observação é influente ou não. Para tal especificação, neste trabalho estaremos considerando a abordagem apresentada por Cook (1986) e a proposta de Billor e Loynes (1993). Portanto, nesta subseção apresentaremos as duas estatísticas que utilizaremos para diferenciar os valores influentes dos demais.

Inicialmente, a análise da superfície de influência será através do conceito de máxima curvatura. Através deste conceito iremos caracterizar se uma observação é influente ou não. Em seguida caracterizaremos uma determinada observação através 
do conceito de máxima inclinação da superfície de influência. Finalmente descrevemos a análise conjunta das estatísticas na constatação da influência de uma dada observação, ou seja, vamos considerar uma observação como influente obedecendo os resultados obtidos através da estatística de máxima curvatura e da estatística de máxima inclinação.

\subsubsection{Curvatura da Superfície de Influência}

O ideal seria podermos vizualizar o gráfico da superfície de influência para um particular modelo e um determinado conjunto de dados. No entanto, isso é possível apenas quando $r$ é menor ou igual a 2. Assim, outros métodos são necessários para obtermos informação do gráfico de influência. Uma aproximação considerada por Cook (1986) foi a análise de influência local, a qual usa a curvatura normal de $\alpha(\omega)$ em $\omega_{0}$.

O procedimento consiste em analisar como a superfície de verossimilhança deslocada desvia-se de um plano tangente em $\omega_{0}$. Isso pode ser feito através das seções normais da curvatura da superfície em questão em um determinado ponto $\omega_{0}$. Se a classe de todas as seções normais da superfície $\alpha(\omega)$ em $\omega_{0}$ tiverem curvatura normal próxima a zero, então $L D(\omega)$ é próxima a zero em $\omega_{0}$, indicando que o modelo sob o determinado esquema de perturbação é estável. Por outro lado, se existir uma seção normal com curvatura em módulo distante de zero, então nessa direção vamos ter maior oscilação da função $L D(\omega)$ e, sob essa seção normal, vamos ter maior influência do particular esquema de perturbação. Se projetarmos esta seção sobre o plano $\Re^{r}$, vamos obter um vetor $\ell \in \Re^{r}$ tal que a reta $\omega_{0}+a \ell, a \in \Re$ e a seção normal pertencem ao mesmo plano. Portanto será equivalente considerar uma seção normal a $\alpha(\omega)$ em $\omega_{0}$ com um vetor $\ell \in \Re^{r}$ para indicar a direção onde existe maior ou menor influência em torno de $\omega_{0}$. 
Calculando-se $L D\left(\omega_{0}+a \ell\right)$ podemos obter uma linha levantada (lifted line) sobre a superfície $\alpha(\omega)$ (que é equivalente a considerar uma seção normal da superfície $\alpha(\omega)$ em $\left.\omega_{0}\right)$. Essa linha levantada é essencialmente a curva plana dada pela parametrização

$$
\rho(a)=\left(\rho_{1}, \rho_{2}\right)^{\prime}=[a, L D(\omega(a))]^{\prime} .
$$

Essa curva pode ser vista como o conjunto de pontos de $\alpha(\omega)$ que são comuns ao plano gerado pelos vetores $\mathbf{v}_{q+1}$ e $\left(\ell^{\prime}, 0\right)^{\prime}$, onde $\mathbf{v}_{q+1}$ é um vetor indicador com valor 1 na coordenada $q+1$ e zero caso contrário. A curvatura normal desta linha, denotada por $C_{\ell}$, é definida como a curvatura da curva $\left[a, L D\left(\omega_{0}+a \ell\right)\right]$, calculada em $\omega_{0}$ (ver Stoker (1969), pg. 26) e definida por

$$
C_{\ell}=\frac{\left|\dot{\rho}_{1} \ddot{\rho}_{2}-\dot{\rho}_{2} \ddot{\rho}_{1}\right|}{\left(\dot{\rho}_{1}^{2}+\dot{\rho}_{2}^{2}\right)^{3 / 2}}
$$

Sob a suposição de que a log-verossimilhança perturbada, $L(\Psi \| \omega)$, é duas vezes diferenciável em $\left(\Psi^{\prime}, \omega^{\prime}\right)^{\prime}$, e portanto, $\rho(l)=[a, L D(\omega(a))]^{\prime}$ também é duas vezes diferenciável, seguem os seguintes resultados:

(i) $\dot{\rho}_{1}=\frac{d a}{d a}=1$ e $\ddot{\rho}_{1}=\frac{d^{2} a}{d a^{2}}=0$;

(ii) $\frac{d \omega(a)}{d a}=\frac{d\left(\omega_{0}+a \ell\right)}{d a}=\ell$ e $\frac{d^{2} \omega(a)}{d a^{2}}=0$;

(iii) $\frac{d L D(\omega(a))}{d a}=\frac{d \omega^{\prime}}{d a} \frac{\partial L D(\omega)}{\partial \omega}=\ell^{\prime} \frac{\partial L D(\omega)}{\partial \omega}=\frac{\partial L D(\omega)}{\partial \omega} \ell$;

(iv) $\dot{\rho}_{2}(0)=\left.\frac{d L D(\omega(a))}{d a}\right|_{a=0}=\left.\left(\left[\frac{\partial L D(\omega)}{\partial \omega}\right]^{\prime} \ell\right)\right|_{\omega=\omega_{0}}=0$, pois $\omega_{0}$ é ponto de mínimo local de $L D(\omega)$. 
Substituindo as derivadas na Equação (5.1.3) temos que

$$
C_{\ell}=\left|\ddot{\rho}_{2}\right|=\mid \ddot{L D}(\omega(a)) \|_{a=0}
$$

Assim, a derivada segunda da verossimilhança deslocada será dada por

$$
\begin{aligned}
\ddot{L D}(\omega(a)) & =\frac{d^{2} L D(\omega(a))}{d a^{2}}=\frac{d}{d a}\left[\frac{d L D(\omega(a))}{d a}\right] \\
& =\frac{d}{d a}\left[\ell^{\prime} \frac{d L D(\omega(a))}{d \omega}\right]=\frac{d}{d a}\left[\left(\frac{d L D(\omega(a))}{d \omega}\right)^{\prime} \ell\right] \\
& =\frac{d}{d a}\left[\frac{d L D(\omega(a))}{d \omega^{\prime}} \ell\right]=\frac{d}{d a}\left[\frac{d L D(\omega(a))}{d \omega^{\prime}}\right] \ell \\
& =\frac{d \omega^{\prime}}{d a} \frac{\partial}{\partial \omega}\left(\frac{\partial L D(\omega(a))}{\partial \omega^{\prime}}\right) \\
& =\ell^{\prime} \frac{\partial^{2} L D(\omega(a))}{\partial \omega \partial \omega^{\prime}} \ell .
\end{aligned}
$$

Quando obtida essa derivada em $a=0$, determinamos a curvatura normal

$$
C_{\ell}=\left|\ell^{\prime} \ddot{L D}\left(\omega_{0}\right) \ell\right|
$$

Mas, pela definição de verossimilhança deslocada, temos que

$$
\ddot{L D}\left(\omega_{0}\right)=\frac{\partial^{2}}{\partial \omega \partial \omega^{\prime}}\left[2\left(L(\hat{\Psi})-L\left(\hat{\Psi}_{\omega}\right)\right)\right]_{\omega=\omega_{0}}=-\left.2 \frac{\partial^{2} L\left(\hat{\Psi}_{\omega}\right)}{\partial \omega \partial \omega^{\prime}}\right|_{\omega=\omega_{0}}
$$

portanto, a curvatura normal é reescrita como

$$
C_{\ell}=2\left|\ell^{\prime} \ddot{F} \ell\right|,
$$


onde $\ddot{F}$ é uma matriz quadrada de ordem $q$, cujos elementos são dados pelas derivadas $\frac{\partial^{2} L\left(\hat{\Psi}_{\omega}\right)}{\partial \omega_{r} \partial \omega_{s}}$, calculadas em $\omega=\omega_{0}$. Essas derivadas parciais cruzadas são dadas por:

$$
\begin{aligned}
\frac{\partial^{2} L\left(\hat{\Psi}_{\omega}\right)}{\partial \omega_{r} \partial \omega_{s}} & =\left.\frac{\partial}{\partial \omega_{r}}\left(\frac{\partial L\left(\hat{\Psi}_{\omega}\right)}{\partial \omega_{s}}\right)\right|_{\omega=\omega_{0}}=\left.\frac{\partial}{\partial \omega_{r}}\left[\left(\frac{\partial L\left(\hat{\Psi}_{\omega}\right)}{\partial \hat{\Psi}_{\omega}}\right)^{\prime} \frac{\partial \hat{\Psi}_{\omega}}{\partial \omega_{s}}\right]\right|_{\omega=\omega_{0}} \\
& =\left.\left\{\frac{\partial}{\partial \omega_{r}}\left(\frac{\partial L\left(\hat{\Psi}_{\omega}\right)}{\partial \hat{\Psi}_{\omega}^{\prime}}\right) \frac{\partial \hat{\Psi}_{\omega}}{\partial \omega_{s}}+\frac{\partial L\left(\hat{\Psi}_{\omega}\right)}{\partial \hat{\Psi}_{\omega}^{\prime}} \frac{\partial}{\partial \omega_{r}}\left(\frac{\partial \hat{\Psi}_{\omega}}{\partial \omega_{s}}\right)\right\}\right|_{\omega=\omega_{0}} \\
& =\left.\left\{\left[\left(\frac{\partial^{2} L\left(\hat{\Psi}_{\omega}\right)}{\partial \hat{\Psi}_{\omega} \partial \hat{\Psi}_{\omega}^{\prime}}\right)^{\prime} \frac{\partial \hat{\Psi}_{\omega}}{\partial \omega_{r}}\right]^{\prime} \frac{\partial \hat{\Psi}_{\omega}}{\partial \omega_{s}}+\frac{\partial L\left(\hat{\Psi}_{\omega}\right)}{\partial \hat{\Psi}_{\omega}^{\prime}} \frac{\partial^{2} \hat{\Psi}_{\omega}}{\partial \omega_{r} \partial \omega_{s}}\right\}\right|_{\omega=\omega_{0}} \\
& =\left.\left\{\frac{\partial \hat{\Psi}_{\omega}}{\partial \omega_{r}^{\prime}} \frac{\partial^{2} L\left(\hat{\Psi}_{\omega}\right)}{\partial \hat{\Psi}_{\omega} \partial \hat{\Psi}_{\omega}^{\prime}} \frac{\partial \hat{\Psi}_{\omega}}{\partial \omega_{s}}+\frac{\partial L\left(\hat{\Psi}_{\omega}\right)}{\partial \hat{\Psi}_{\omega}^{\prime}} \frac{\partial^{2} \hat{\Psi}_{\omega}}{\partial \omega_{r} \partial \omega_{s}}\right\}\right|_{\omega=\omega_{0}} .
\end{aligned}
$$

Como, por hipótese, $L\left(\Psi \| \omega_{0}\right)=L(\Psi)$, para todo $\Psi \in \Re^{p}$, tem-se $\hat{\Psi}_{\omega_{0}}=\hat{\Psi}$, portanto

$$
\left.\frac{\partial L\left(\hat{\Psi}_{\omega}\right)}{\partial \hat{\Psi}_{\omega}}\right|_{\omega=\omega_{0}}=\left.\frac{\partial L\left(\Psi \| \omega_{0}\right)}{\partial \Psi}\right|_{\Psi=\hat{\Psi}}=0
$$

já que é ponto de máximo da função de log-verossimilhança, tanto no modelo postulado $L(\Psi)$, quanto no modelo perturbado $L\left(\Psi \| \omega_{0}\right)$. Com isso, a segunda parcela da derivada da Equação (5.1.4) é nula. Logo,

$$
\frac{\partial^{2} L\left(\hat{\Psi}_{\omega}\right)}{\partial \omega_{r} \partial \omega_{s}}=\left.\left[\left(\frac{\partial \hat{\Psi}_{\omega}}{\partial \omega_{r}}\right)^{\prime}\left(\frac{\partial^{2} L\left(\hat{\Psi}_{\omega}\right)}{\partial \hat{\Psi}_{\omega} \partial \hat{\Psi}_{\omega}^{\prime}}\right)\left(\frac{\partial \hat{\Psi}_{\omega}}{\partial \omega_{s}}\right)\right]\right|_{\omega=\omega_{0}} ; r, s=1, \ldots, q
$$

Reescrevendo de forma matricial temos

$$
\ddot{F}=J^{\prime} \ddot{L} J
$$

$\operatorname{com} J^{\prime}=\frac{\partial \hat{\Psi}_{\omega}}{\partial \omega_{r}}$ e $\ddot{L}=\frac{\partial^{2} L\left(\hat{\Psi}_{\omega}\right)}{\partial \hat{\Psi}_{\omega} \partial \hat{\Psi}_{\omega}^{\prime}}$ calculadas em $\omega=\omega_{0}$. A fim de obtermos uma expressão para $J$ vamos derivar a Equação (5.1.4) em relação a $\omega$ em $\omega_{0}$. 


$$
\begin{aligned}
\left.\frac{\partial}{\partial \omega}\left(\frac{\partial L\left(\hat{\Psi}_{\omega} \| \omega\right)}{\partial \Psi^{\prime}}\right)\right|_{\omega=\omega_{0}} & =\left.\left\{\frac{\partial \hat{\Psi}_{\omega}^{\prime}}{\partial \omega} \frac{\partial^{2} L\left(\hat{\Psi}_{\omega} \| \omega\right)}{\partial \hat{\Psi}_{\omega} \partial \hat{\Psi}^{\prime}}+\frac{\partial \omega^{\prime}}{\partial \omega} \frac{\partial^{2} L\left(\hat{\Psi}_{\omega} \| \omega\right)}{\partial \omega \partial \Psi^{\prime}}\right\}\right|_{\omega=\omega_{0}} \\
& =\left.\left\{\frac{\partial \hat{\Psi}_{\omega}^{\prime}}{\partial \omega} \frac{\partial^{2} L\left(\hat{\Psi}_{\omega} \| \omega\right)}{\partial \hat{\Psi}_{\omega} \partial \hat{\Psi}^{\prime}}+\frac{\partial^{2} L\left(\hat{\Psi}_{\omega} \| \omega\right)}{\partial \omega \partial \Psi^{\prime}}\right\}\right|_{\omega=\omega_{0}}
\end{aligned}
$$

Observe que

$$
\left.\frac{\partial^{2} L\left(\hat{\Psi}_{\omega} \| \omega\right)}{\partial \hat{\Psi}_{\omega} \partial \hat{\Psi}^{\prime}}\right|_{\omega=\omega_{0}}=\frac{\partial^{2} L\left(\hat{\Psi}_{\omega_{0}} \| \omega_{0}\right)}{\partial \hat{\Psi}_{\omega_{0}} \partial \hat{\Psi}^{\prime}}=\frac{\partial^{2} L(\hat{\Psi})}{\partial \hat{\Psi} \partial \hat{\Psi}^{\prime}}=\left.\frac{\partial^{2} L(\hat{\Psi})}{\partial \Psi \partial \Psi^{\prime}}\right|_{\Psi=\hat{\Psi}}
$$

e assim, temos que

$\left.\frac{\partial}{\partial \omega}\left(\frac{\partial L\left(\hat{\Psi}_{\omega} \| \omega\right)}{\partial \Psi^{\prime}}\right)\right|_{\omega=\omega_{0}}=\left.\left.\frac{\partial \hat{\Psi}_{\omega}^{\prime}}{\partial \omega}\right|_{\omega=\omega_{0}} \frac{\partial^{2} L(\hat{\Psi})}{\partial \Psi \partial \Psi^{\prime}}\right|_{\Psi=\hat{\Psi}}+\left.\frac{\partial^{2} L(\Psi \| \omega)}{\partial \omega \partial \Psi^{\prime}}\right|_{\omega=\omega_{0}, \Psi=\hat{\Psi}}=J^{\prime} \ddot{F}+\Delta^{\prime}=0$ com $\Delta=\left.\frac{\partial^{2} L(\Psi \| \omega)}{\partial \omega \partial \Psi^{\prime}}\right|_{\omega=\omega_{0}, \Psi=\hat{\Psi}}$, ou seja, $J^{\prime}=-\delta \ddot{F}^{-1}$. Agora, voltando a $\ddot{F}$ obtemos:

$$
\begin{aligned}
\ddot{F} & =\left.\left.\left.\left(\frac{\partial \hat{\Psi}_{\omega}^{\prime}}{\partial \omega}\right)\right|_{\omega=\omega_{0}}\left(\frac{\partial^{2} L\left(\hat{\Psi}_{\omega}\right)}{\partial \hat{\Psi}_{\omega} \partial \hat{\Psi}_{\omega}^{\prime}}\right)\right|_{\Psi=\hat{\Psi}}\left(\frac{\partial \hat{\Psi}_{\omega}}{\partial \omega}\right)^{\prime}\right|_{\omega=\omega_{0}} \\
& =\left(-\Delta^{\prime} \ddot{L}^{-1}\right) \ddot{L}\left(-\Delta^{\prime} \ddot{L}^{-1}\right)^{\prime} \\
& =\Delta^{\prime} \ddot{L}^{-1} \Delta
\end{aligned}
$$

e dessa forma Cook (1986) mostrou que para um vetor unitário $\ell \in \Omega$,

$$
C_{\ell}=2\left|\ell^{\prime} \Delta^{\prime} \ddot{L}^{-1} \Delta \ell\right|
$$

Existem diversas formas pelo qual a Equação (5.1.5) pode ser usada para estudar $\alpha(\omega)$, dependendo da escolha do vetor unitário $\ell$. Uma escolha correspondendo a 
perturbação na $i$-ésima posição é obtida tomando-se $\ell_{i}$, que é o valor de $\ell$ na posição $i$ como sendo igual a zero, e zero nas outras posições. O resultado da influência local será dado por

$$
C_{i}=C_{\ell_{i}}=2\left|\Delta_{i}^{\prime} \ddot{L}^{-1} \Delta_{i}\right|
$$

Uma das formas de se estudar a superfície $\alpha(\omega)$ é através do extremo, $C_{\max }$, que é o máximo autovalor absoluto de $-\Delta^{\prime} \ddot{L}^{-1} \Delta$ e $\ell_{\max }=\left(\ell_{1_{\max }}, \ldots, \ell_{n_{\max }}\right)^{\prime}$ o autovetor associado. A direção de $\ell_{\max }$ é a direção que a curvatura é maximizada. Isso mostra como perturbar o modelo postulado de forma a obter maiores mudanças locais na verossimilhança deslocada. Se, por exemplo, o $i$-ésimo componente de $\ell_{\max }$ é grande, isso indica que a perturbação na $i$-ésima observação pode produzir distorções significantes nos resultados da análise.

Vale lembrar que o procedimento dado tem como objetivo entender o comportamento de um gráfico de influência em torno de $\omega_{0}$. Cook (1986) apresentou a curvatura normal de uma forma onde analisamos o gráfico de influência em qualquer outro ponto $\omega^{*}$. Assim, ele considerou a curvatura dada por

$$
C_{\ell}=\frac{\ell^{\prime} \ddot{F} \ell}{\left[1+\dot{F}^{\prime} \dot{F}\right]^{1 / 2} \ell^{\prime}\left[I+\dot{F} \dot{F}^{\prime}\right] \ell^{\prime}}
$$

onde

$$
\begin{aligned}
& \dot{F}=\frac{2 \partial L(\Psi \| \omega)}{\partial \omega}, \\
& \ddot{F}=2\left\{\frac{\partial^{2} L(\Psi \| \omega)}{\partial \omega \partial \omega^{\prime}}-\left[\frac{\partial^{2} L(\Psi \| \omega)}{\partial \Psi \partial \omega^{\prime}}\right]^{\prime}\left[\frac{\partial^{2} L(\Psi \| \omega)}{\partial \Psi \partial \Psi^{\prime}}\right]^{-1} \frac{\partial^{2} L(\Psi \| \omega)}{\partial \Psi \partial \omega^{\prime}}\right\}
\end{aligned}
$$


com as derivadas calculadas em $\omega_{0}$ e $\hat{\Psi}$. O vetor direção da máxima curvatura normal pode indicar influência local de perturbações no modelo postulado. Dessa forma, Cook (1986) e Wu e Luo (1993) verificaram que a curvatura normal é maximizada em $\omega=\omega_{0}$ na direção do autovetor associado ao maior autovalor da equação característica $|A-\lambda B|=0$ onde $A=\ddot{F}$ e $B=\left(1+\dot{F}^{\prime} \dot{F}\right)^{0.5}\left(I+\dot{F} \dot{F}^{\prime}\right)$. Portanto, para a verificarmos a existência de alguma observação influente será considerada a máxima curvatura $C_{M a x}$. Depois usa-se o autovetor, $c_{\text {Max }}=\left(c_{1}, \ldots, c_{n}\right)^{\prime}$, associado a curvatura significante para localizarmos qual observação é influente.

\subsubsection{Inclinação da Superfície de Influência}

Billor e Loynes (1993) analisaram a superfície de influência através da verossimilhança deslocada modificada. Eles propuseram analisar a influência considerando a máxima inclinação, $S_{M a x}$, e sua direção correspondente, $\ell_{\text {Max }}$. Como apenas derivadas de primeira ordem estão envolvidas, essa abordagem é mais simples que a análise através de curvatura apresentada por Cook (1986). A análise se dá através do cálculo do gradiente de $L D^{*}(\omega)$, e assim temos

$$
S_{M a x}=\left|\nabla L D^{*}\left(\omega_{0}\right)\right|
$$

Note que

$$
\frac{\partial L D^{*}}{\partial a}=\ell^{\prime} \frac{\partial L D^{*}}{\partial \omega}=2 \ell^{\prime}\left[\frac{\partial L\left(\hat{\Psi}_{\omega} \| \omega\right)}{\partial \omega}+\frac{\partial L(\Psi \| \omega)}{\partial \Psi} \frac{\partial \hat{\Psi}_{\omega}}{\partial \omega}\right]
$$

quando calculado em $\hat{\Psi}$ e $\omega_{0}$, reduz-se a 


$$
\left.2 \ell^{\prime} \frac{\partial L(\Psi \| \omega)}{\partial \omega}\right|_{\Psi=\hat{\Psi}, \omega=\omega_{0}}
$$

Portanto,

$$
S_{M a x}=2|\nabla L(\hat{\Psi} \| \omega)|
$$

e temos que a direção, $s_{\text {Max }}$, tem coseno dado por

$$
\cos \theta_{i}=\frac{\frac{\partial L D^{*}}{\partial \omega_{i}}}{\left|\nabla L D^{*}\right|} i=1, \ldots, n .
$$

Portanto, utilizaremos a análise da máxima inclinação, $S_{M a x}$ para verificar a existência de valores influentes. Após detectarmos a presença de valores influentes, verificamos quais observações são influentes através da análise da direção associada a esta inclinação, $s_{\text {Max }}=\left(s_{1} \ldots, s_{n}\right)^{\prime}$, onde $s_{i}=\cos \theta_{i}$. Nas próximas seções apresentaremos como proceder na identificação de valores influentes, através da análise desas duas estatísticas associadas à análise da superfície de influência.

\subsection{Procedimento para Determinação de Valores Influentes}

Na seção anterior foram definidos os seguintes testes estatísticos: $S_{\text {Max }}$, e $s_{\text {Max }}=$ $\left(s_{1}, \ldots, s_{n}\right)^{\prime}, C_{M a x}$, e $c_{M a x}=\left(c_{1}, \ldots, c_{n}\right)^{\prime}$, onde estas estatísticas são definidas para cada tipo de perturbação. Nesta seção apresentaremos dois procedimentos para testar valores influentes baseadas nestas estatísticas. Estes procedimentos são sugeridos no trabalho de Zhang e King (2005), mas apenas o primeiro procedimento foi de 
fato utilizado por Zhang e King (2005) e é adotado nas aplicações apresentadas na seção 5.4. Para cada tipo de perturbação o primeiro procedimento é realizado em dois passos enquanto o segundo procedimento é dado em apenas 1 passo.

\section{Procedimento A:}

Passo 1: Utilizando as estatísticas $S_{M a x}$ e $C_{M a x}$ teste se existe algum observação influente para o tipo de perturbação sob consideração. Considera-se que existe alguma observação influente se alguma estatística for significante estatisticamente. Se isto ocorre passe ao passo 2; caso contrário para-se o teste não se rejeitando a hipótese nula de inexistência de valores influentes. Zhang e King (2005) sugerem utilizar nível de significância de $5 \%$ neste passo.

Passo 2: Para verificar quais observações são influentes utilizam-se os vetores diagnósticos $s_{\text {Max }}$ e $c_{\text {Max }}$. A $i$-ésima observação é considerada influente segundo o diagnóstico baseado na inclinação (curvatura) se $s_{i}\left(c_{i}\right)$ for maior do que um valor crítico. Zhang e King (2005) sugerem que se utilize como valor crítico o percentil $5 \%$ da distribuição de $\max \left\{s_{1}, \ldots, s_{n}\right\}\left(\max \left\{c_{1}, \ldots, c_{n}\right\}\right)$ para a inclinação (curvatura), condicionado ao fato de que $S_{\text {Max }}$ ou $C_{M a x}$ foi significante no primeiro passo.

Procedimento B: Neste procedimento utiliza-se apenas os vetores diagnósticos. A $i$ ésima observação é considerada influente segundo o diagnóstico baseado na inclinação (curvatura) se $s_{i}\left(c_{i}\right)$ for maior do que um valor crítico. Só que diferentemente do passo 2 do procedimento anterior utiliza-se a distribuição não condicionada de $\max \left\{s_{1}, \ldots, s_{n}\right\}\left(\max \left\{c_{1}, \ldots, c_{n}\right\}\right)$ para a inclinação (curvatura).

Na subseção 5.4.1 será apresentado o procedimento para estimar os valores críticos necessários para a utilização destes procedimentos. 


\subsection{Influência Local em Modelos de VE}

Agora, apresentamos os resultados necessários para estender a metodologia para o modelo VE. Serão considerados vários tipos de perturbações e os cálculos necessários para o desenvolvimento da curvatura e inclinação, que serão fundamentais nas análises gráficas de influência local.

Como vimos na subseção 5.2, precisamos encontrar a máxima curvatura e máxima inclinação. Portanto, é necessário encontrar os valores de $\dot{F}$ e $\ddot{F}$ dadas pela Equação 5.1.8. Vale ressaltar que esses valores também são suficientes para o cálculo da máxima inclinação.

Pode-se notar pela Equação 5.1.8 que precisamos encontrar derivadas da verossimilhança, $L(\Psi \mid \omega)$, para o cálculo da curvatura e inclinação.

É de conhecimento na literatura que a verossimilhança para o modelo VE não pode ser obtida de forma analítica. A partir do trabalho de Harvey et al. (1994), muitas técnicas têm sido desenvolvidas a fim de melhorar os resultados das aproximações e obter a verossimilhança "exata". Em nosso trabalho consideramos o cálculo da verossimilhança pelo método proposto por Durbin e Koopman (1997), onde eles utilizam amostragem por importância em conjunto com o filtro de Kalman para obter a verossimilhança.

Nas próximas seções apresentaremos inicialmente o cálculo da verossimilhança "exata" para o modelo de volatilidade estocástica. Obtida a verossimilhança o desenvolvimento necessário para os cálculos da curvatura e inclinação são apresentados sob os tipos de perturbações considerados. 


\subsubsection{Cálculo da Verossimilhança}

Nesta seção mostraremos como calculamos a verossimilhança para o modelo de volatilidade estocástica.

Inicialmente, assuma que o modelo de Volatilidade Estocástica postulado seja dado como segue:

$$
\begin{aligned}
& y_{t}=\beta e^{h_{t} / 2} \epsilon_{t}, \quad t=1, \ldots, n, \\
& h_{t}=\mu+\phi h_{t-1}+\sigma_{\eta} \eta_{t},
\end{aligned}
$$

onde $\epsilon_{t}$ e $\eta_{t}$ possuem média zero e variância unitária. Nesses termos, o modelo de volatilidade pode ser reescrito em uma forma mais geral como proposto em Durbin e Koopman (1997) (DK), onde é dado por:

$$
\begin{aligned}
y_{t} & \sim p\left(y_{t} \mid \theta_{t}\right) & \theta_{t} & =h_{t} \\
h_{t} & =T_{t} h_{t-1}+d_{t}+D_{t} \eta_{t} & \eta_{t} & \sim N I D(0, I) .
\end{aligned}
$$

Seja $\mathbf{y}=\left(y_{1}^{\prime}, \ldots, y_{n}^{\prime}\right)^{\prime}, h=\left(h_{1}^{\prime}, \ldots, h_{n}^{\prime}\right)^{\prime}$. Assim pode-se denotar as densidades como segue:

- $p(\mathbf{y})$ - Densidade marginal de $\mathbf{y}$.

- $p(\theta)$ - Densidade marginal de $\theta$.

- $p(\mathbf{y}, \theta)$ - Densidade conjunta de $\mathbf{y}$ e $\theta$.

- $p(\mathbf{y} \mid \theta)$ - Densidade de $\mathbf{y}$ dado $\theta$,

onde todas essas densidades são para um dado conjunto de parâmetros $\Psi$. Assim a função de verossimilhança é dada da seguinte forma, 


$$
\begin{aligned}
L(\Psi) & =p(\mathbf{y}) \\
& =\int p(\mathbf{y}, \theta) d \theta \\
& =\int p(\mathbf{y} \mid \theta) p(\theta) d \theta .
\end{aligned}
$$

$L(\Psi)$ pode ser estimada através de simulação, escolhedo $N$ retiradas independentes de $\theta^{(1)}, \ldots, \theta^{(N)}$ da distribuição com densidade $p(\theta)$ e tomando-se a média dos valores de $p\left(\mathbf{y} \mid \theta^{(i)}\right)$ para $i=1, \ldots, N$. A eficiência deste procedimento pode ser melhorada através de técnicas tais como amostragem por importância, variável antitética e variável controle.

DK propuseram o método de amostragem por importância considerando uma densidade, a qual será chamada de densidade importante, $\widetilde{p}(\theta \mid \mathbf{y})$ uma densidade condicional, de $\theta$ dado y e para um certo $\Psi$, da qual podem ser retiradas amostras mais facilmente. Assim

$$
\begin{aligned}
L(\Psi) & =\int p(y \mid \theta) p(\theta) d \theta \\
& =\int p(y \mid \theta) \frac{p(\theta)}{\widetilde{p}(\theta \mid \mathbf{y})} \widetilde{p}(\theta \mid \mathbf{y}) d \theta \\
& =\widetilde{E}\left\{p(y \mid \theta) \frac{p(\theta)}{\widetilde{p}(\theta \mid \mathbf{y})}\right\} .
\end{aligned}
$$

Fazendo-se $N$ retiradas de $\theta^{(i)}$ da densidade $\widetilde{p}(\theta \mid \mathbf{y})$ pode-se estimar $L(\Psi)$ por :

$$
\hat{L}(\Psi)=\frac{1}{N} \sum_{i=1}^{N} p\left(\mathbf{y} \mid \theta^{(i)}\right) \frac{p\left(\theta^{(i)}\right)}{\widetilde{p}\left(\theta^{(i)} \mid \mathbf{y}\right)}
$$


Dada uma amostra $\theta^{(i)} \sim \widetilde{p}(\theta \mid \mathbf{y}) \operatorname{com} \widetilde{p}(\theta \mid \mathbf{y}) \sim N(\hat{\theta}, V)$ obtida pelo algoritmo de suavização proposto por Jungbacker e Koopman (2006), $\hat{\theta}$ é a moda de $p(\theta \mid \mathbf{y})$. Jungbacker e Koopman (2006) demonstraram que os parâmetros $\hat{\theta}$ e $V$ podem ser obtidos através de um processo iterativo a partir do filtro de Kalman padrão sem consideração de linearidade da equação de observação $p\left(y_{t} \mid \theta\right)$. O denominador $\widetilde{p}\left(\theta^{(i)} \mid \mathbf{y}\right)$ é obtido por

$$
\widetilde{p}\left(\theta^{(i)} \mid \mathbf{y}\right)=\exp \left(-\frac{n}{2} \log 2 \pi-\sum_{t=1}^{n} \log \left|A_{t}\right|-\sum_{t=1}^{n} \log \left|B_{t}\right|-\frac{1}{2} \sum_{t=1}^{n} \nu_{t}^{i^{\prime}} \nu_{t}^{i}\right),
$$

onde $\nu_{t}^{i} \sim N\left(0, I_{m}\right), A_{t}$ e $B_{t}$ são obtidas através do algoritmo de suavização proposto por Jungbacker e Koopman (2006). A obtenção da Equação (5.3.5) é dada no Apêndice do artigo de Jungbalker e Koopman (2006).

Para o modelo de volatilidade estocástica considerado neste trabalho temos que $\log p(\mathbf{y} \mid \theta)=\sum_{t=1}^{n} \log p\left(y_{t} \mid \theta_{t}\right)$ onde

$$
\log p\left(y_{t} \mid \theta_{t}\right)=-\frac{1}{2} \log (2 \pi)-\frac{1}{2} h_{t}-\frac{1}{2} \frac{y_{t}^{2}}{e^{h_{t}}}
$$

Obtidas $\widetilde{p}(\theta \mid \mathbf{y}) p(\mathbf{y} \mid \theta)$ podemos encontrar a verossimilhança para o modelo em estudo. Devemos considerar os esquemas de perturbações que estaremos analisando através da metodologia de influência. Portanto, definiremos alguns tipos de perturbações considerados na literatura.

No trabalho vamos considerar a perturbação dos dados e do modelo. A perturbação no modelo é importante pois, geralmente os modelos estatísticos são aproximações das descrições de processos complicados, e portanto, temos diversas suposições envolvidas em uma determinada análise. Por exemplo, em um modelo de 
regressão linear simples, supomos que o erro tem média zero, variância constante e são não correlacionados com distribuição normal.

Por outro lado, erros de medidas e outliers podem influenciar os resultados das estimativas e assim verificar influência das perturbações nos dados torna-se necessária. Billor e Loynes (1993) assim como Zhang e King (2005) consideraram as duas formas de perturbações nos modelos analisados. Neste trabalho estaremos considerando a perturbação dos dados e perturbação no modelo. Nessa última serão consideradas a perturbação aditiva e a perturbação na volatilidade. 


\subsubsection{Perturbação das Observações}

A partir do modelo postulado dado pela Equação (5.3.1) é feita a extensão de forma a considerarmos a perturbação nas observações. Suponha que observamos os dados com perturbações $\omega=\left(\omega_{1}, \cdots, \omega_{n}\right)^{\prime}$, e o vetor de perturbação nula, $\omega_{0}$, é um vetor $n \times 1$ de zeros. Assim, teremos o modelo considerado dado como segue,

$$
y_{t}+\omega_{t}=e^{\frac{h_{t}}{2}} \epsilon_{t}, \quad t=1, \ldots, n
$$

Agora, para aplicar a metodologia apresentada na seção 5.2 devemos encontrar algumas derivadas da verossimilhança. Como visto, no caso do modelo de volatilidade estocástica a verossimilhança foi encontrada por métodos numéricos. Portanto, iremos apresentar os resultados necessários para obter as derivadas da Equação (5.3.4), e assim precisamos das derivadas de $p(\mathbf{y} \mid \theta)$ e $\widetilde{p}(\theta \mid \mathbf{y})$. Onde temos que, para o modelo com perturbação nas observações, essas funções são dadas por:

$$
\begin{gathered}
\log p(\mathbf{y} \mid \theta)=-\frac{n}{2} \log (2 \pi)-\frac{1}{2} \sum_{t=1}^{n} h_{t}-\frac{1}{2} \sum_{t=1}^{n} \frac{\left(y_{t}+\omega_{t}\right)^{2}}{e^{h_{t}}} \\
\log \widetilde{p}\left(\theta^{(i)} \mid \mathbf{y}\right)=-\frac{n}{2} \log 2 \pi-\sum_{t=1}^{n} \log \left|A_{t}\right|-\sum_{t=1}^{n} \log \left|B_{t}\right|-\frac{1}{2} \sum_{t=1}^{n} \nu_{t}^{i^{\prime}} \nu_{t}^{i} .
\end{gathered}
$$

Para analisarmos a superfície de influência, encontraremos a curvatura $C_{\ell}$ definida

pela Equação (5.1.7). Para isso devemos encontrar $\dot{F}$ e $\ddot{F}$, portanto considere as derivadas necessárias para o cálculo da curvatura normal dadas por: 


$$
\begin{aligned}
\frac{\partial p(\mathbf{y} \mid \theta)}{\partial \Psi} & =-\frac{1}{2 \sqrt{2 \pi}} \sum_{t=1}^{n}\left\{\frac{e^{-h_{t}-\frac{\left(y_{t}+\omega_{t}\right)^{2}}{2 e^{h_{t}}}}}{\sqrt{e^{h_{t}}}}-\frac{e^{h_{t}-\frac{\left(y_{t}+\omega_{t}\right)^{2}}{2 e^{h_{t}}}}}{e^{1.5 h_{t}}}\right\} \frac{\partial h_{t}}{\partial \Psi} \\
\frac{\partial^{2} p(\mathbf{y} \mid \theta)}{\partial \Psi \partial \Psi^{\prime}} & =\sum_{t=1}^{n}\left\{\frac{3 e^{2 h_{t}-\frac{\left(y_{t}+\omega_{t}\right)^{2}}{2 e^{h_{t}}}}}{4 e^{2.5 h_{t} \sqrt{2 \pi}}}-\frac{\left(y_{t}+\omega_{t}\right)^{2} e^{-\frac{\left(y_{t}+\omega_{t}\right)^{2}}{2 e^{h_{t}}}}}{4 e^{1.5 h_{t} \sqrt{2 \pi}}}\right\} \frac{\partial h_{t}}{\partial \Psi} \frac{\partial h_{t}}{\partial \Psi^{\prime}} \\
& +\left\{\frac{e^{h_{t}-\frac{\left(y_{t}+\omega_{t}\right)^{2}}{2 e^{h t}}}}{2 e^{1.5 h_{t} \sqrt{2 \pi}}}-\frac{\left(y_{t}+\omega_{t}\right)^{2} e^{h_{t}-\frac{\left(y_{t}+\omega_{t}\right)^{2}}{2 e^{h t}}}}{2 e^{0.5 h_{t} \sqrt{2 \pi}}}\right\}\left\{\frac{\partial h_{t}}{\partial \Psi}\left[\frac{\partial h_{t}}{\partial \Psi^{\prime}}+\frac{\left(y_{t}+\omega_{t}\right)^{2}}{2 e^{h_{t}}} \frac{\partial h_{t}}{\partial \Psi^{\prime}}\right]\right\} \\
\frac{\partial p(\mathbf{y} \mid \theta)}{\partial \omega} & =-\sum_{t=1}^{n} \frac{\left(y_{t}+\omega_{t}\right)}{\sqrt{2 \pi}} e^{-\frac{3 h_{t}}{2}-\frac{\left(y_{t}+\omega_{t}\right)^{2}}{2 e x p\left(h_{t}\right)}} \frac{\partial \omega_{t}}{\partial \omega} \\
\frac{\partial^{2} p(\mathbf{y} \mid \theta)}{\partial \omega \partial \omega^{\prime}} & =\sum_{t=1}^{n} \frac{1}{\sqrt{2 \pi e^{h_{t}}}}\left\{\left(y_{t}+\omega_{t}\right)^{2} e^{-2 h_{t}-\frac{\left(y_{t}+\omega_{t}\right)^{2}}{2 e_{t}^{h}}}-e^{-h_{t}-\frac{\left(y_{t}+\omega_{t}\right)^{2}}{2 e_{t}^{h}}}\right\} \frac{\partial \omega_{t}}{\partial \omega} \frac{\partial \omega_{t}}{\partial \omega^{\prime}} \\
\frac{\partial^{2} p(\mathbf{y} \mid \theta)}{\partial \Psi \partial \omega^{\prime}} & =\sum_{t=1}^{n} \frac{\left(y_{t}+\omega_{t}\right)}{2 \sqrt{2 \pi e^{h_{t}}}}\left\{3 e^{-h_{t}-\frac{\left(y_{t}+\omega_{t}\right)^{2}}{2 e^{h_{t}}}}-\left(y_{t}+\omega_{t}\right) e^{-2 h_{t}-\frac{\left(y_{t}+\omega_{t}\right)^{2}}{2 e^{h_{t}}}}\right\} \frac{\partial h_{t}}{\partial \Psi} \frac{\partial \omega_{t}}{\partial \omega^{\prime}}
\end{aligned}
$$

calculadas em $\omega_{0}$. As derivadas para o cálculo de $\dot{F}$ e $\ddot{F}$ são dadas por:

$$
\begin{aligned}
&\left.\frac{\partial p(\mathbf{y} \mid \theta)}{\partial \Psi}\right|_{\omega=\omega_{0}}=-\frac{1}{2 \sqrt{2 \pi}} \sum_{t=1}^{n}\left\{\frac{e^{-h_{t}-\frac{y_{t}^{2}}{2 e^{h_{t}}}}}{\sqrt{e^{h_{t}}}}-\frac{e^{h_{t}-\frac{y_{t}^{2}}{2 e^{h_{t}}}}}{e^{1.5 h_{t}}}\right\} \frac{\partial h_{t}}{\partial \Psi} \\
&\left.\frac{\partial^{2} p(\mathbf{y} \mid \theta)}{\partial \Psi \partial \Psi^{\prime}}\right|_{\omega=\omega_{0}}=\sum_{t=1}^{n}\left\{\frac{3 e^{2 h_{t}-\frac{y_{t}^{2}}{2 e^{h_{t}}}}}{4 e^{2.5 h_{t} \sqrt{2 \pi}}}-\frac{y_{t}^{2} e^{-\frac{y_{t}^{2}}{2 e^{h_{t}}}}}{4 e^{1.5 h_{t} \sqrt{2 \pi}}}\right\} \frac{\partial h_{t}}{\partial \Psi} \frac{\partial h_{t}}{\partial \Psi^{\prime}} \\
&+\left\{\frac{e^{h_{t}-\frac{y_{t}^{2}}{2 e^{h_{t}}}}}{2 e^{1.5 h_{t} \sqrt{2 \pi}}}-\frac{y_{t}^{2} e^{h_{t}-\frac{y_{t}^{2}}{2 e^{h_{t}}}}}{2 e^{0.5 h_{t} \sqrt{2 \pi}}}\right\}\left\{\frac{\partial h_{t}}{\partial \Psi}\left[\frac{\partial h_{t}}{\partial \Psi^{\prime}}+\frac{y_{t}^{2}}{2 e^{h_{t}}} \frac{\partial h_{t}}{\partial \Psi^{\prime}}\right]\right\} \\
&\left.\frac{\partial p(\mathbf{y} \mid \theta)}{\partial \omega}\right|_{\omega=\omega_{0}}=\left.\sum_{t=1}^{n} \frac{y_{t}}{\sqrt{2 \pi} e^{-\frac{3 h_{t}}{2}-\frac{y_{t}^{2}}{2 e x p\left(h_{t}\right)}} \frac{\partial \omega_{t}}{\partial \omega}}\right|_{\omega=\omega_{0}}=\sum_{t=1}^{n} \frac{1}{\sqrt{2 \pi e^{h_{t}}}}\left\{y_{t}^{2} e^{-2 h_{t}-\frac{y_{t}^{2}}{2 e_{t}^{h}}}-e^{-h_{t}-\frac{y_{t}^{2}}{2 e_{t}^{h}}}\right\} \frac{\partial \omega_{t}}{\partial \omega} \frac{\partial \omega_{t}}{\partial \omega^{\prime}} \\
&\left.\frac{\partial^{2} p(\mathbf{y} \mid \theta)}{\partial \Psi \partial \omega^{\prime}}\right|_{\omega=\omega_{0}}=\sum_{t=1}^{n} \frac{y_{t}}{2 \sqrt{2 \pi e^{h_{t}}}}\left\{3 e^{-h_{t}-\frac{y_{t}^{2}}{2 e^{h_{t}}}}-y_{t} e^{\left.-2 h_{t}-\frac{y_{t}^{2}}{2 e^{h_{t}}}\right\} \frac{\partial h_{t}}{\partial \Psi} \frac{\partial \omega_{t}}{\partial \omega^{\prime}} .}\right.
\end{aligned}
$$

Verifique que no modelo de Volatilidade Estocástica as derivadas da volatilidade em relação a perturbação aditiva é igual a zero. Portanto, devemos encontrar apenas as 
derivadas $\partial h_{t} / \partial \Psi$, que são dadas por

$$
\frac{\partial h_{t}}{\partial \Psi}=\left[\begin{array}{c}
\frac{\partial h_{t}}{\partial \phi} \\
\frac{\partial h_{t}}{\partial \sigma_{\eta}}
\end{array}\right]=\left[\begin{array}{c}
\phi^{t} \frac{\partial h_{0}}{\partial \phi}+\sum_{i=0}^{t-1} \phi^{i} h_{t-1-i} \\
\phi^{t} \frac{\partial h_{0}}{\partial \sigma_{\eta}}+\sum_{i=0}^{t-1} \phi^{i} \eta_{t-i}
\end{array}\right] .
$$

Lembremos que $h_{t}$ é não observável. Portanto para obtermos as derivadas será utilizado as estimativas pela média encontradas a partir do SsfPack. Neste pacote Koopman et al.(1998) implementaram o algoritmo de suavizamento proposto por de Jong e Shephard (1995) de forma a utilizar o filtro de Kalman para estimar a volatilidade. Portanto, podemos obter estimativas de $h_{t}$ e $\eta_{t}$ através desse algoritmo, considerando o ponto inicial para $\frac{\partial h_{0}}{\partial \Psi}$ estimativas do estado $h_{0}$, que será considerado como a média de uma amostra obtida a partir das primeiras 50 estimativas de $h_{t}$ obtidas através do algoritmo de suavização.

Vamos encontrar as derivadas da Equação (5.3.8), onde os termo $A_{t}$ e $B_{t}$ são obtidos através do algoritmo de suavização proposto por Jungbacker e Koopman (2006). No algoritmo proposto pode-se observar que $B_{t}$ é função das observações através de $A_{t}$. Portanto a função resume-se a

$$
\log \widetilde{p}\left(\theta^{(i)} \mid \mathbf{y}\right) \propto-\sum_{t=1}^{n} \log \left|A_{t}\right|-\sum_{t=1}^{n} \log \left|f\left(A_{t}^{-1}\right)\right| .
$$

Dada a natureza do problema, isto é, encontrar a moda da densidade $p(\mathbf{y} \mid \theta)$, pode-se provar que $A_{t}=\frac{\partial^{2} \log p\left(y_{t} \mid \theta_{t}\right)}{\partial \theta \partial \theta^{\prime}}$ e assim voltamos ao problema de encontrar as derivadas similares àquelas dadas em (5.3.9). 


\subsubsection{Perturbação do Modelo}

Nesta seção serão considerados dois tipos de perturbação do modelo: perturbações no nível e perturbações na volatilidade. No primeiro caso será apresentada perturbação local para o modelo e posteriormente através da perturbação na volatilidade consideramos a propragação da perturbação através da estrutura autoregressiva do modelo.

\subsubsection{Perturbação Aditiva}

O vetor de perturbação é introduzido no modelo adicionando perturbação ao nível dos erros padronizados,

$$
\epsilon_{t}+\omega_{t} \sim N(0,1)
$$

Sob esse modelo, a função necessária para o cálculo das derivadas da verossimilhança do modelo sob esse esquema de perturbação, será dada por:

$$
\log p(\mathbf{y} \mid \theta)=-\frac{n}{2} \log (2 \pi)-\frac{1}{2} \sum_{t=1}^{n} h_{t}-\frac{1}{2} \sum_{t=1}^{n}\left(\frac{y_{t}}{e^{h_{t} / 2}}+\omega_{t}\right)^{2}
$$

Portanto, as derivadas necessárias para o cálculo parcial de $\dot{F}$ e $\ddot{F}$ são obtidas através de métodos numéricos como citado na subseção anterior. 


\subsubsection{Perturbação na Volatilidade}

Agora, apresentaremos o modelo onde consideramos a pertubação no modelo de volatilidade estocástica através de influências na volatilidade. A forma generalizada do modelo com perturbação na volatilidade é dado por:

$$
\begin{aligned}
y_{t} & =\beta e^{h_{t}(\omega) / 2} \epsilon_{t}, \quad t=1, \ldots, n \\
h_{t}(\omega) & =\phi h_{t-1}(\omega)+\sigma_{\eta} \eta_{t}+\omega_{t} .
\end{aligned}
$$

Neste caso, $y_{t}$ condicionado a $h_{t}$ tem distribuição normal com média zero e variância $e^{h_{t}(\omega)}$. Sob esse esquema de perturbação, uma das funções necessárias para estimarmos a verossimilhança a fim de obter a curvatura, é dada por :

$$
\log p(\mathbf{y} \mid \theta)=-\frac{n}{2} \log (2 \pi)-\frac{1}{2} \sum_{t=1}^{n} h_{t}(\omega)-\frac{1}{2} \sum_{t=1}^{n} \frac{y_{t}^{2}}{e^{h_{t}(\omega)}}
$$

Para analisarmos a superfície de influência, encontraremos a curvatura $C_{\ell}$ definida pela Equação (5.1.7). Para o cálculo de $\log \widetilde{p}\left(\theta^{(i)} \mid y_{t}\right)$ o procedimento é análogo ao considerado anteriormente.

Para isso devemos encontrar $\dot{F}$ e $\ddot{F}$. Portanto considere as derivadas necessárias para o cálculo da curvatura normal obtidas por método numérico. 
O modelo com perturbação na volatilidade será representado por:

$$
h_{t}(\omega)=\phi h_{t-1}(\omega)+\sigma_{\eta} \eta_{t}-\omega_{t}
$$

$$
\begin{aligned}
\frac{\partial h_{t}(\omega)}{\partial \sigma_{\eta}} & =\eta \\
\frac{\partial^{2} h_{t}(\omega)}{\partial \sigma_{\eta} \partial \phi} & =\frac{\partial^{2} h_{t}(\omega)}{\partial \sigma_{\eta} \partial \omega_{j}}=0, \\
\frac{\partial h_{t}(\omega)}{\partial \omega_{j}} & =\phi \frac{\partial h_{t-1}(\omega)}{\partial \omega_{j}}-\frac{\partial \omega_{t}}{\partial \omega_{j}}, \\
\frac{\partial h_{t}(\omega)}{\partial \omega_{t+k}} & =-\phi^{k} I_{(k \geq 0)}, \\
\frac{\partial^{2} h_{t}(\omega)}{\partial \omega_{j} \partial \sigma_{\eta}} & =\frac{\partial^{2} h_{t-1}(\omega)}{\partial \omega_{j} \partial \omega_{j}^{\prime}}=0, \\
\frac{\partial^{2} h_{t}(\omega)}{\partial \omega_{j+k} \partial \phi} & =-k \phi^{k-1} I_{(k \geq 1)} .
\end{aligned}
$$

Obtidas as derivadas parciais, através das devidas substituições nas Equações (5.1.7) e (5.1.11) encontraremos a máxima curvatura e máxima inclinação e seus respectivos vetores direcionais associados.

Na seção 5.4 serão obtidos limiares (pontos críticos) de modo a caracterizar uma observação influente. Após estudos baseados em simulações, o método será aplicado às duas séries reais em estudo. 


\subsection{Aplicação da Análise de Influência Local}

Nesta seção serão consideradas as análises das séries de retornos do índice da bolsa de valores do Estado de São Paulo e NYSE. Inicialmente utilizaremos os limiares por valores críticos, para realizar os testes necessários para utilizar o procedimento descrito na seção 5.2. As aplicações são apresentadas na subseção 5.4.1.

\subsubsection{Estimativas dos Valores Limiares}

As estimativas são obtidas através da distribuição empírica da máxima inclinação e curvatura. Dessa forma foram simuladas um total de $N=1000$ replicações de tamanhos $T=500,1000$ e 1500 . O conjunto de parâmetros utilizado para simular as séries foi obtido através do ajuste do modelo de volatilidade a série de retornos da NYSE e Ibovespa. O modelo foi ajustado utilizando-se a verossimilhança exata obtida pela metodologia apresentada na seção 5.3.1.

Portanto, em cada replicação da simulaçao, foram utilizados os parâmetros estimados para gerar amostra e encontrar a máxima inclinação e máxima curvatura, assim como os vetores direcionais associados.

Para cada uma das 1000 séries foram encontrados os valores de $C_{\max }$ e $S_{\max }$ obtendo-se assim 1000 valores de curvatura máxima $\left\{C_{\max _{1}}, \ldots, C_{\max _{N}}\right\}$ e máxima inclinação $\left\{S_{\max _{1}}, \ldots, S_{\max _{N}}\right\}$. Para cada um desses valores temos um autovetor associado, logo temos 1000 autovetores de tamanho $T, c=\left\{c_{1}, \ldots, c_{T}\right\}$ para máxima curvatura e $s=\left\{s_{1}, \ldots, s_{T}\right\}$ para máxima inclinção, onde $T$ depende do tamanho da série considerada. Inicialmente vamos obter o que será considerado limiar para as curvaturas e inclinação, ou seja, a partir dos percentis da distribuição empírica da curvatura $C_{\max }$, e inclinação, $S_{\max }$, caracterizaremos um ponto de corte. 
Na Tabela 5.1 encontram-se as estimativas dos percentis da máxima curvatura e inclinação, necessários para o Passo 1 do primeiro procedimento. Neste passo realizamos um teste geral para verificar a existência de valores influentes.

\begin{tabular}{|c|c|c|c|c|c|c|c|}
\hline \multirow[b]{3}{*}{ Amostra } & \multirow[b]{3}{*}{ Percentis } & \multicolumn{4}{|c|}{ Perturbação do Modelo } & \multirow{2}{*}{\multicolumn{2}{|c|}{ Perturbação nos Dados }} \\
\hline & & \multicolumn{2}{|c|}{ Volatilidade } & \multicolumn{2}{|c|}{ Aditiva } & & \\
\hline & & Inclinação & Curvatura & Inclinação & Curvatura & Inclinação & Curvatura \\
\hline \multirow{5}{*}{500} & 99.0 & 1263.31 & 128.42 & 1512.43 & 177.86 & 2794.26 & 137.73 \\
\hline & 97.5 & 1253.00 & 95.05 & 1367.81 & 148.92 & 2736.89 & 105.02 \\
\hline & 95.0 & 1150.19 & 87.72 & 1179.09 & 78.66 & 2675.08 & 93.99 \\
\hline & 90.0 & 1118.57 & 75.25 & 1050.80 & 65.12 & 2562.15 & 83.26 \\
\hline & 99.0 & 2254.76 & 135.92 & 4908.32 & 198.81 & 5168.26 & 148.00 \\
\hline \multirow{3}{*}{1000} & 97.5 & 2215.46 & 112.36 & 4879.61 & 182.97 & 4709.91 & 125.30 \\
\hline & 95.0 & 2154.49 & 96.48 & 4653.73 & 157.25 & 4503.12 & 116.08 \\
\hline & 90.0 & 2015.33 & 89.41 & 4635.47 & 101.81 & 4345.31 & 101.65 \\
\hline \multirow{4}{*}{1500} & 99.0 & 3314.19 & 154.35 & 6395.28 & 240.95 & 7381.62 & 171.42 \\
\hline & 97.5 & 3219.56 & 147.36 & 6364.35 & 225.32 & 6939.87 & 160.90 \\
\hline & 95.0 & 3141.65 & 134.78 & 6243.43 & 174.45 & 6633.55 & 153.39 \\
\hline & 90.0 & 3117.43 & 129.82 & 6202.81 & 159.31 & 6517.34 & 135.29 \\
\hline
\end{tabular}

Tabela 5.1: Estimativas dos percentis da máxima curvatura e inclinação.

Se em uma dada perturbação for detectada a presença de valor localmente influente em um dos testes, de máxima curvatura ou máxima inclinação, passamos ao Passo 2, onde devemos encontrar qual das observações são influentes sob o esquema de perturbação em estudo.

A Tabela 5.2 apresenta as características distribucionais dos maiores componentes em um vetor diagnóstico, que já foi detectado como influente pela curvatura ou inclinação ao nível de $5 \%$, ou seja, usando os limiares apresentados na Tabela 5.1. Dados os vetores diagnósticos influentes e seus maiores componentes associados, utilizamos o percentil de $5 \%$ como limiar para localizar observações influentes. Esses limiares são usados para detectar observações influentes após a identificação, pela curvatura ou inclinação, do vetor diagnóstico como influente através Tabela 5.1.

Pode-se notar que o valor da estimativa do quantil utilizado para determinação 


\begin{tabular}{|c|c|c|c|c|c|c|c|}
\hline \multirow[b]{3}{*}{ Amostra } & \multirow[b]{3}{*}{ Percentis } & \multicolumn{4}{|c|}{ Perturbação do Modelo } & \multirow{2}{*}{\multicolumn{2}{|c|}{ Perturbação nos Dados }} \\
\hline & & \multicolumn{2}{|c|}{ Volatilidade } & \multicolumn{2}{|c|}{ Aditiva } & & \\
\hline & & Inclinação & Curvatura & Inclinação & Curvatura & Inclinação & Curvatura \\
\hline \multirow{5}{*}{500} & Máximo & 0.622 & 0.713 & 0.176 & 0.340 & 0.231 & 0.353 \\
\hline & Mínimo & 0.027 & 0.025 & 0.051 & 0.041 & 0.013 & 0.019 \\
\hline & $5 \%$ Quantil & 0.249 & 0.384 & 0.148 & 0.135 & 0.121 & 0.170 \\
\hline & Média & 0.431 & 0.482 & 0.163 & 0.207 & 0.201 & 0.234 \\
\hline & Desvio & 0.059 & 0.091 & 0.010 & 0.043 & 0.203 & 0.021 \\
\hline \multirow{5}{*}{1000} & Máximo & 0.543 & 0.708 & 0.141 & 0.327 & 0.213 & 0.324 \\
\hline & Mínimo & 0.014 & 0.023 & 0.024 & 0.019 & 0.008 & 0.012 \\
\hline & $5 \%$ Quantil & 0.184 & 0.298 & 0.111 & 0.128 & 0.115 & 0.145 \\
\hline & Média & 0.387 & 0.431 & 0.123 & 0.218 & 0.227 & 0.188 \\
\hline & Desvio & 0.048 & 0.112 & 0.079 & 0.031 & 0.113 & 0.019 \\
\hline \multirow{5}{*}{1500} & Máximo & 0.504 & 0.668 & 0.134 & 0.256 & 0.198 & 0.272 \\
\hline & Mínimo & 0.004 & 0.020 & 0.018 & 0.050 & 0.001 & 0.010 \\
\hline & $5 \%$ Quantil & 0.179 & 0.202 & 0.093 & 0.121 & 0.072 & 0.127 \\
\hline & Média & 0.342 & 0.416 & 0.119 & 0.164 & 0.137 & 0.148 \\
\hline & Desvio & 0.036 & 0.101 & 0.062 & 0.025 & 0.105 & 0.018 \\
\hline
\end{tabular}

Tabela 5.2: Propriedades distribucionais do maior componente do vetor diagnóstico significante classificados na Tabela 5.1 .

do ponto limiar decresce conforme o tamanho da série aumenta. Portanto, como a série da NYSE possui 1255 observações, na análise será considerada a interpolação entre o quantil obtido considerando amostra de tamanho $T=1000$ e $T=1500$.

Com o objetivo de verificar se os valores críticos dependem fortemente dos valores dos parâmetros do modelo, foi realizado uma simulação com 500 replicações a partir dos parâmetros estimados para a série da NYSE. A Tabela 5.3 apresenta os pontos críticos quando consideramos a interpolação pela Tabela 5.2 e quando simulamos a partir dos valores de seus parâmetros. Visto que os valores são relativamente próximos, podemos utilizar os valores críticos obtidos através da interpolação para analisar a série do NYSE e utilizar os valores dos parâmetros estimados para estimar os dados de referência.

No caso da série de retornos do Ibovespa utilizamos o próprio valor do quantil dado na Tabela 5.2 porque o tamanho da série é igual ao tamanho das séries simuladas consideradas para obtermos os limiares. 


\begin{tabular}{|c|c|c|c|c|c|c|c|}
\hline \multirow[b]{3}{*}{ Parâmetros } & \multirow[b]{3}{*}{ Percentis } & \multicolumn{4}{|c|}{ Perturbação do Modelo } & \multirow{2}{*}{\multicolumn{2}{|c|}{ Perturbação nos Dados }} \\
\hline & & \multicolumn{2}{|c|}{ Volatilidade } & \multicolumn{2}{|c|}{ Aditiva } & & \\
\hline & & Inclinação & Curvatura & Inclinação & Curvatura & Inclinação & Curvatura \\
\hline \multirow{4}{*}{ Ibovespa } & Máximo & 0.5235 & 0.6880 & 0.1375 & 0.2915 & 0.3010 & 0.2980 \\
\hline & Mínimo & 0.0090 & 0.0215 & 0.0210 & 0.0345 & 0.0045 & 0.0110 \\
\hline & $5 \%$ Quantil & 0.1815 & 0.2600 & 0.1020 & 0.1245 & 0.0935 & 0.1360 \\
\hline & Média & 0.3645 & 0.4235 & 0.1210 & 0.1910 & 0.1820 & 0.1680 \\
\hline \multirow{4}{*}{ NYSE } & Máximo & 0.534 & 0.6987 & 0.1415 & 0.3038 & 0.3193 & 0.3102 \\
\hline & Mínimo & 0.010 & 0.0232 & 0.0226 & 0.0356 & 0.0051 & 0.0127 \\
\hline & $5 \%$ Quantil & 0.195 & 0.2625 & 0.1099 & 0.1256 & 0.1019 & 0.1419 \\
\hline & Média & 0.377 & 0.4318 & 0.1213 & 0.2149 & 0.1971 & 0.1793 \\
\hline
\end{tabular}

Tabela 5.3: Comparação das propriedades distribucionais para os modelos estimados para as séries do Ibovespa e NYSE dos maiores componentes do vetor diagnóstico significante classificados na Tabela 5.1 .

Agora, consideraremos o Procedimento B descrito na seção 5.2, que consiste de um único passo, onde devemos encontrar um limiar para caracterizarmos a partir dos autovetores associados a máxima inclinação e máxima curvatura estatisticamente significantes, quais observações são influentes.

Para isso analisaremos as características relevantes da distribuição empírica dos vetores diagnóstico, que são os autovetores associados às estatísticas de máxima curvatura e máxima inclinação. Dados os 1000 autovetores associados a máxima curvatura $c=\left\{c_{1}, \ldots, c_{T}\right\}$ e máxima inclinação $s=\left\{s_{1}, \ldots, s_{T}\right\}$, vamos considerar os valores da maior componente de cada autovetor, ou seja, teremos um conjunto de dados com 1000 observações que são os maiores componentes de cada vetor diagnóstico.

No procedimento B utilizaremos os percentis dados na Tabela 5.4. Assim, a observação associada a um valor do vetor diagnóstico maior que, por exemplo, o percentil 95\% será considerada um outlier sob um determinado esquema de perturbação.

Visto que caracterizamos uma observação extrememente influente, outlier, precisamos analisar quando uma observação é influente. Neste caso, vamos considerar os autovetores associados as máximas curvaturas e máximas inclinações considerados 


\begin{tabular}{|c|c|c|c|c|c|c|c|}
\hline \multirow[b]{3}{*}{ Amostra } & \multirow[b]{3}{*}{ Percentis } & \multicolumn{4}{|c|}{ Perturbação do Modelo } & \multirow{2}{*}{\multicolumn{2}{|c|}{ Perturbação nos Dados }} \\
\hline & & \multicolumn{2}{|c|}{ Volatilidade } & \multicolumn{2}{|c|}{ Aditiva } & & \\
\hline & & Inclinação & Curvatura & Inclinação & Curvatura & Inclinação & Curvatura \\
\hline \multirow{4}{*}{500} & 99.0 & 0.5523 & 0.6888 & 0.1746 & 0.3381 & 0.2292 & 0.3525 \\
\hline & 97.5 & 0.5054 & 0.6388 & 0.1676 & 0.3127 & 0.2220 & 0.3483 \\
\hline & 95.0 & 0.4630 & 0.5951 & 0.1613 & 0.2967 & 0.2080 & 0.3471 \\
\hline & 90.0 & 0.4400 & 0.5402 & 0.1548 & 0.2695 & 0.1973 & 0.3106 \\
\hline \multirow{4}{*}{1000} & 99.0 & 0.5371 & 0.6111 & 0.1391 & 0.2933 & 0.2128 & 0.3031 \\
\hline & 97.5 & 0.5326 & 0.6014 & 0.1387 & 0.2691 & 0.2106 & 0.2740 \\
\hline & 95.0 & 0.5214 & 0.5818 & 0.1379 & 0.2437 & 0.2013 & 0.2592 \\
\hline & 90.0 & 0.5003 & 0.5329 & 0.1315 & 0.2219 & 0.1891 & 0.2372 \\
\hline \multirow{4}{*}{1500} & 99.0 & 0.4849 & 0.5151 & 0.1300 & 0.2560 & 0.1876 & 0.2695 \\
\hline & 97.5 & 0.4536 & 0.4514 & 0.1245 & 0.2288 & 0.1862 & 0.2446 \\
\hline & 95.0 & 0.4290 & 0.4050 & 0.1218 & 0.2103 & 0.1725 & 0.2256 \\
\hline & 90.0 & 0.4016 & 0.3713 & 0.1169 & 0.1943 & 0.1536 & 0.2087 \\
\hline
\end{tabular}

Tabela 5.4: Percentis dos maiores componentes dos vetores diagnóstico da máxima curvatura e inclinação.

influentes pela Tabela 5.1.

Na próxima subseção serão apresentadas as análises das séries reais consideradas neste trabalho. Inicialmente o método será aplicado a série de retornos da NYSE e posteriormente utilizaremos a série do Ibovespa. 


\subsubsection{Análise da Série Ibovespa}

Nesta subseção o método proposto neste capítulo é aplicado a série do índice da bolsa de valores do estado de São Paulo. A Figura 5.2 apresenta os retornos da série do índice bovespa que estamos considerando no decorrer deste trabalho.

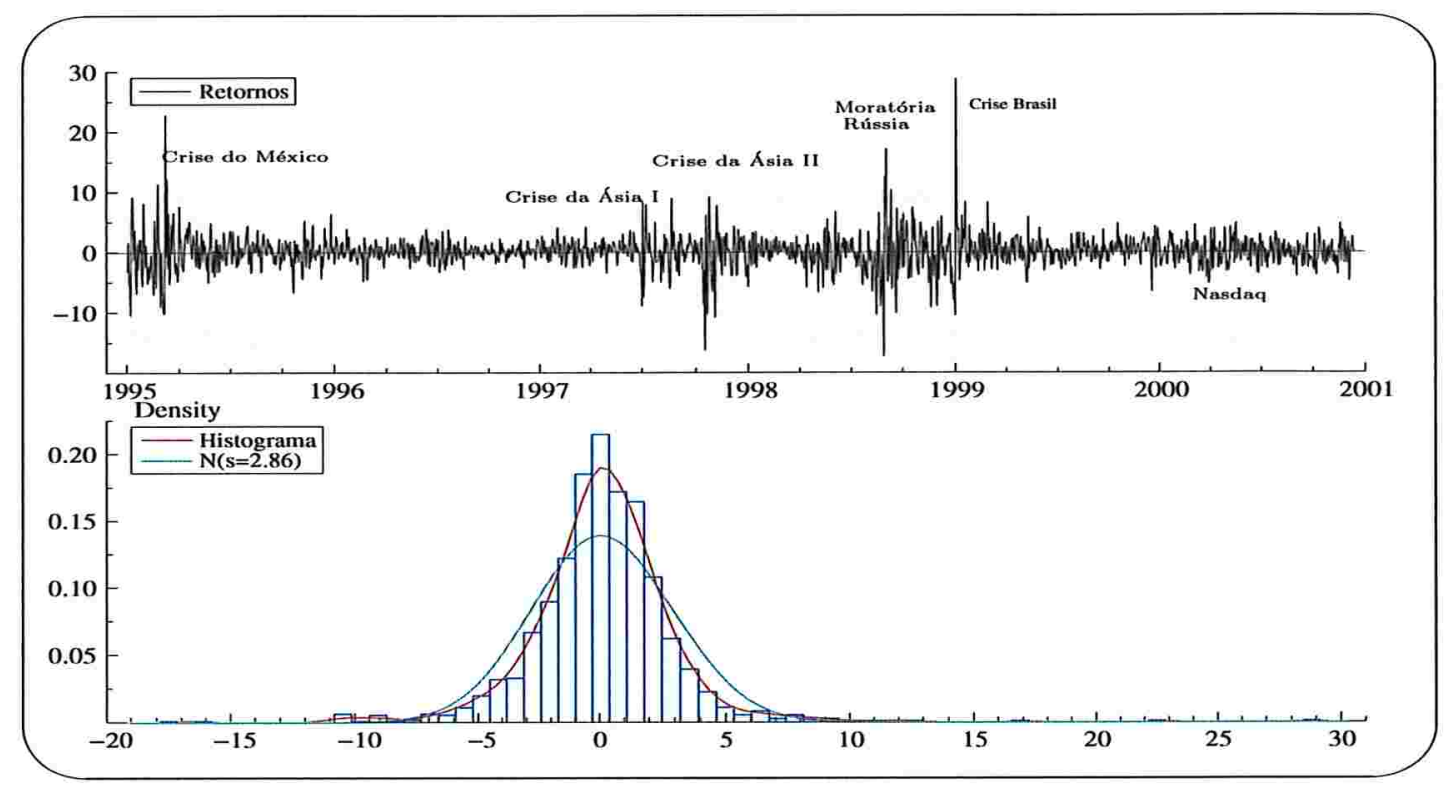

Figura 5.2: Gráfico dos retornos e histograma do Ibovespa.

Na Figura 5.3 encontramos o vetor diagnóstico obtido através da estatística de máxima curvatura e o valor do limiar dado na seção anterior de modo a caracterizarmos um valor influente. A Figura 5.4 apresenta o vetor direção associado a estatística de máxima inclinação. 


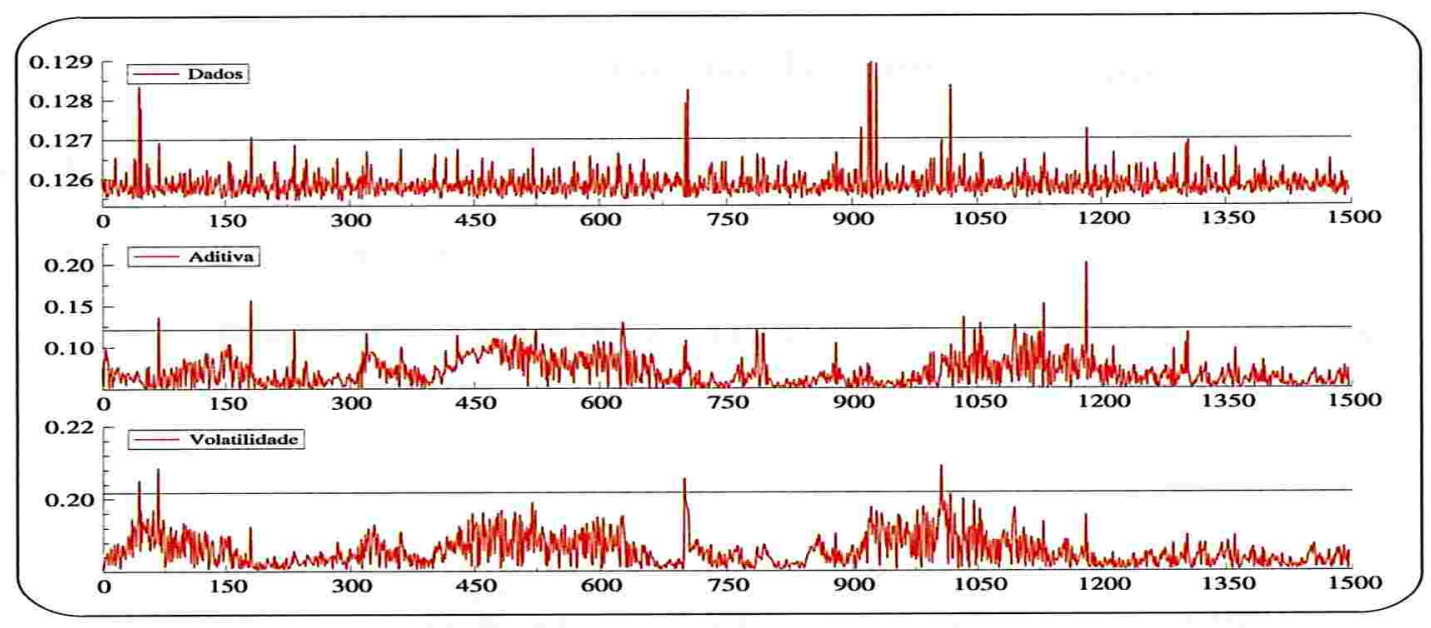

Figura 5.3: Gráfico dos vetores diagnóstico para curvatura do Ibovespa

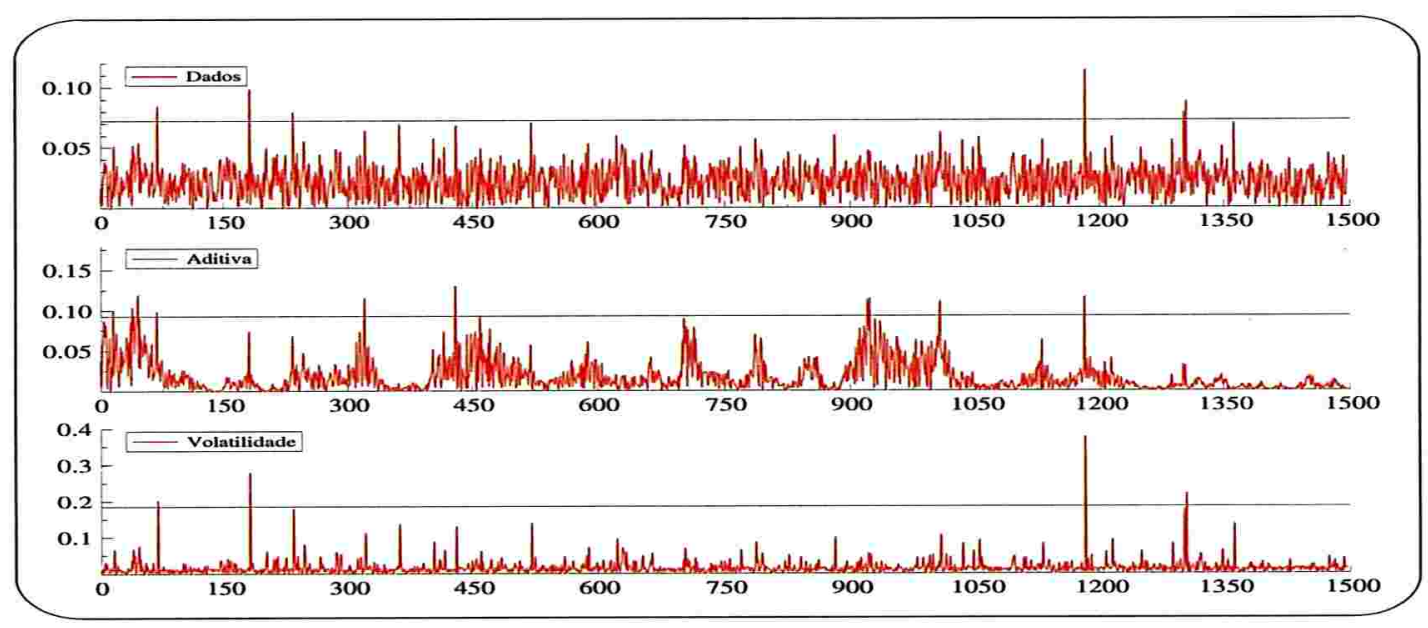

Figura 5.4: Gráfico dos vetores diagnóstico para inclinação do Ibovespa 
A Tabela 5.5 apresenta os valores da série de retornos do Ibovespa que foram caracterizados como influentes. Na tabela podemos verificar a data de ocorrência assim como a localização na ilustração dada nas figuras anteriormente. Apresentamos os valores obtidos das estatísticas propostas na data de localização dos valores caracterizados como influentes por pelo menos uma das estatísticas propostas, considerando todos os três esquemas de perturbação apresentados.

\begin{tabular}{|c|c|c|c|c|c|c|c|}
\hline \multirow[b]{3}{*}{ Data } & \multirow[b]{3}{*}{ Localização } & \multicolumn{4}{|c|}{ Perturbação do Modelo } & \multirow{2}{*}{\multicolumn{2}{|c|}{ Perturbação nos Dados }} \\
\hline & & \multicolumn{2}{|c|}{ Volatilidade } & \multicolumn{2}{|c|}{ Aditiva } & & \\
\hline & & Inclinação & Curvatura & Inclinação & Curvatura & Inclinação & Curvatura \\
\hline $08 / 03 / 1995$ & 45 & 0.0315 & 0.2051 & 0.1193 & 0.0601 & 0.0389 & 0.1283 \\
\hline $10 / 03 / 1995$ & 47 & 0.0082 & 0.1882 & 0.0912 & 0.0582 & 0.0142 & 0.1278 \\
\hline $10 / 04 / 1995$ & 68 & 0.0042 & 0.2085 & 0.0989 & 0.1373 & 0.0248 & 0.1257 \\
\hline $11 / 04 / 1995$ & 69 & 0.2030 & 0.1874 & 0.0274 & 0.0555 & 0.0848 & 0.1269 \\
\hline $25 / 09 / 1995$ & 182 & 0.0069 & 0.1850 & 0.0737 & 0.1568 & 0.0156 & 0.1258 \\
\hline $26 / 09 / 1995$ & 183 & 0.2793 & 0.1822 & 0.0027 & 0.0716 & 0.0986 & 0.1271 \\
\hline $18 / 04 / 1996$ & 321 & 0.0204 & 0.1891 & 0.1151 & 0.1177 & 0.0339 & 0.1256 \\
\hline $20 / 09 / 1996$ & 430 & 0.0026 & 0.1909 & 0.1302 & 0.1149 & 0.0196 & 0.1258 \\
\hline $22 / 10 / 1997$ & 702 & 0.0377 & 0.2054 & 0.0335 & 0.0689 & 0.0415 & 0.1255 \\
\hline $23 / 10 / 1997$ & 703 & 0.0013 & 0.1991 & 0.0896 & 0.1084 & 0.0207 & 0.1279 \\
\hline $28 / 10 / 1997$ & 706 & 0.0181 & 0.1954 & 0.0788 & 0.0817 & 0.0327 & 0.1282 \\
\hline $25 / 08 / 1998$ & 912 & 0.0058 & 0.1849 & 0.0769 & 0.0728 & 0.0258 & 0.1273 \\
\hline $08 / 09 / 1998$ & 921 & 0.0088 & 0.1945 & 0.1131 & 0.0770 & 0.0135 & 0.1289 \\
\hline $09 / 09 / 1998$ & 922 & 0.0552 & 0.1933 & 0.0989 & 0.0663 & 0.0480 & 0.1273 \\
\hline $11 / 09 / 1998$ & 924 & 0.0143 & 0.1899 & 0.1144 & 0.0624 & 0.0308 & 0.1289 \\
\hline $21 / 09 / 1998$ & 930 & 0.0142 & 0.1948 & 0.0890 & 0.0558 & 0.0014 & 0.1289 \\
\hline $13 / 01 / 1999$ & 1008 & 0.0426 & 0.2089 & 0.1109 & 0.0905 & 0.0434 & 0.1270 \\
\hline $28 / 01 / 1999$ & 1019 & 0.0046 & 0.2022 & 0.0487 & 0.1024 & 0.0179 & 0.1283 \\
\hline $22 / 02 / 1999$ & 1034 & 0.0051 & 0.1888 & 0.0228 & 0.1355 & 0.0175 & 0.1258 \\
\hline $13 / 07 / 1999$ & 1131 & 0.0143 & 0.1854 & 0.0628 & 0.1514 & 0.0001 & 0.1260 \\
\hline $23 / 09 / 1999$ & 1182 & 0.0033 & 0.1823 & 0.1158 & 0.2001 & 0.0241 & 0.1257 \\
\hline $24 / 09 / 1999$ & 1183 & 0.3752 & 0.1877 & 0.0200 & 0.0594 & 0.1135 & 0.1272 \\
\hline $17 / 03 / 2000$ & 1303 & 0.1752 & 0.1841 & 0.0169 & 0.0689 & 0.0792 & 0.1269 \\
\hline $22 / 03 / 2000$ & 1306 & 0.2197 & 0.1849 & 0.0064 & 0.0671 & 0.0880 & 0.1270 \\
\hline Máximo & & 5722.2 & 130.37 & 6321.5 & 326.5 & 7233.1 & 143.29 \\
\hline$T_{0}$ & & 3141.6 & 134.78 & 6243.4 & 225.3 & 6939.9 & 160.90 \\
\hline$T_{1}$ & & 0.1792 & 0.2021 & 0.0930 & 0.1213 & 0.0722 & 0.1271 \\
\hline$T_{2}$ & & 0.4290 & 0.4050 & 0.1218 & 0.2103 & 0.3321 & 0.2256 \\
\hline
\end{tabular}

Tabela 5.5: Diagnóstico baseado na curvatura e inclinação para Ibovespa.

Na Tabela 5.5, máximo refere-se a máxima inclinação e curvatura sob cada esquema de perturbação. $T_{0}$ é o limiar da máxima inclinação ou máxima curvatura ao n.s. de 5\%. $T_{1}$ é o limiar obtido através dos limiares do tamanho de amostra 1500 
na Tabela 5.2, e $T_{2}$ é o limiar obtido pela tabela 5.4. Os maiores componentes do vetor diagnóstico estão em negrito, e suas observações correpondentes são identificadas como influentes.

Pode-se notar que o método detectou como influentes as crises de intrabandas, Rússia, a crise mundial das bolsas e a desvalorização do real. Veja que, a estatística de curvatura sob a perturbação das observações foi a que detectou maior número de observações influentes. 


\subsubsection{Análise da Série NYSE}

A série de retornos da NYSE será analisada através dos gráficos dos vetores diagnósticos obtidos através do método proposto. A Figura 5.5 apresenta a série de retornos do índice em estudo. Na Figura 5.6 podemos verificar o comportamento do vetor diagnóstico obtido através da estatística de máxima curvatura.

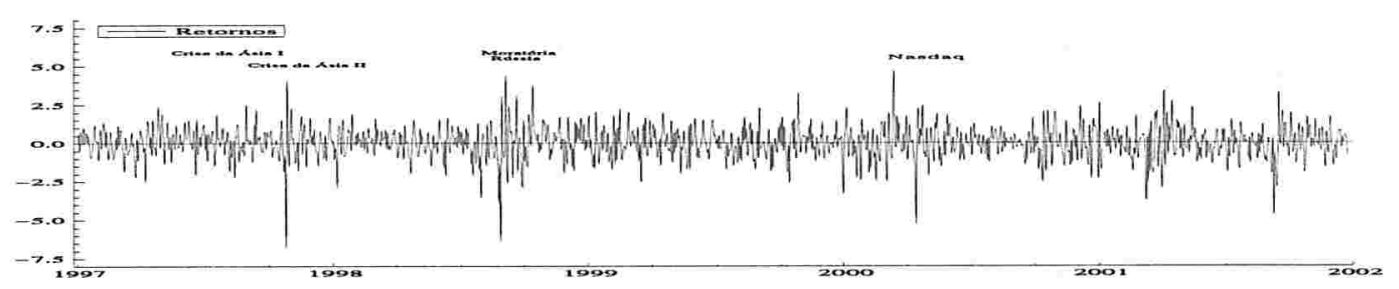

Figura 5.5: Gráfico da série e retornos da NYSE.

A Figura 5.7 apresenta o vetor direção associado a estatística de máxima inclinação. Na Tabela 5.6 apresentamos os valores dos vetores diagnósticos, tanto para a estatística de máxima curvatura quanto para máxima inclinação, pois analisaremos conjuntamente os resultados obtidos a fim de caracterizarmos a influência de uma determinada observação. 


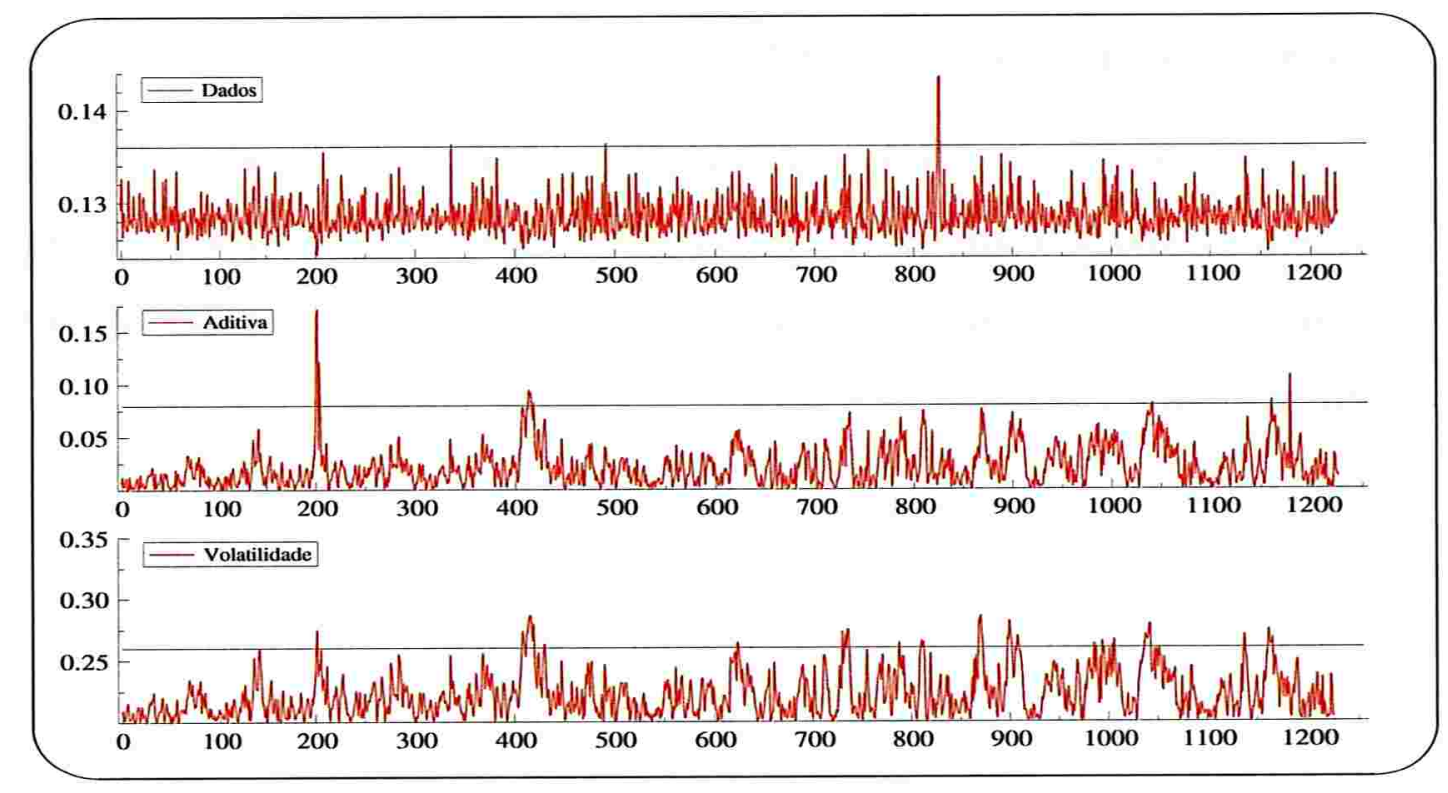

Figura 5.6: Gráfico dos vetores diagnóstico para curvatura da NYSE

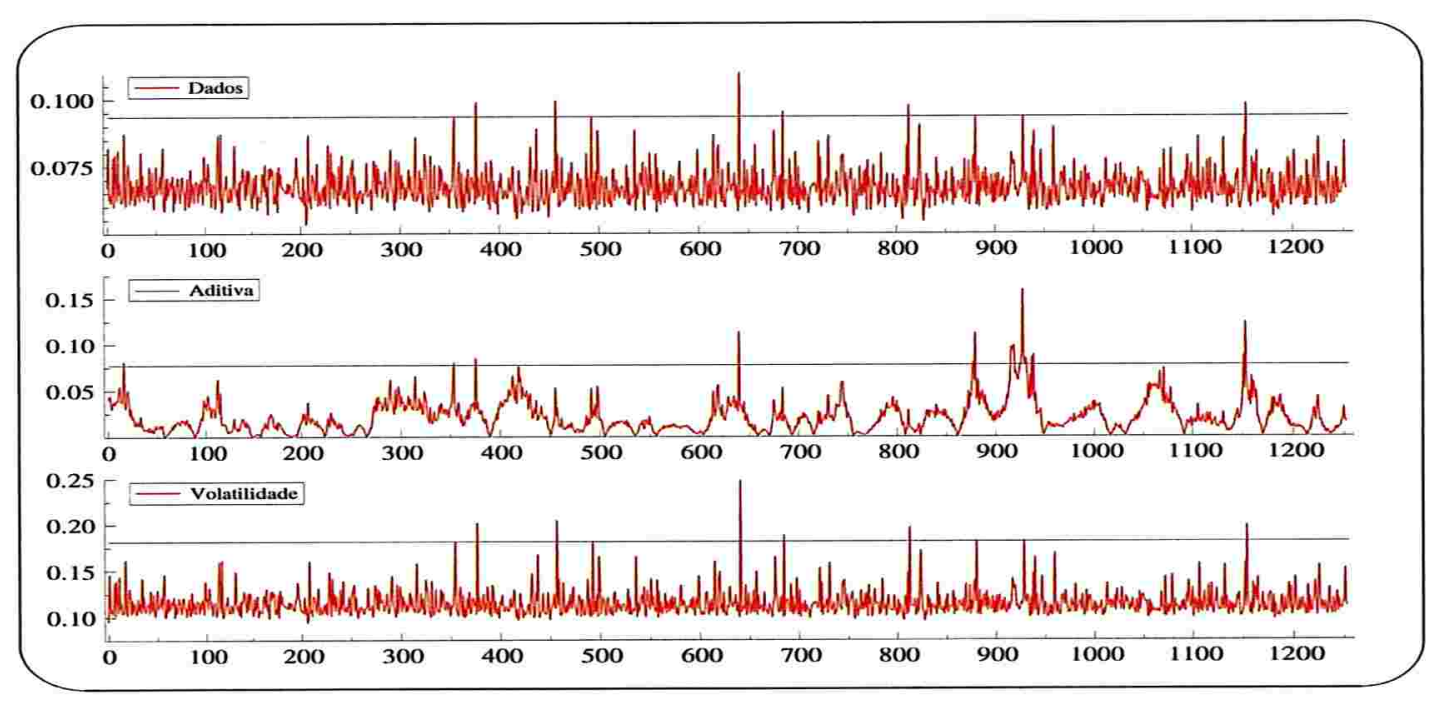

Figura 5.7: Gráfico dos vetores diagnóstico para inclinação do NYSE 
A Tabela 5.6 apresenta os valores da série de retornos do NYSE que foram caracterizados como influentes. Na tabela podemos verificar a data de ocorrência assim como a localização na ilustração dada nas figuras anteriormente.

São apresentadas apenas as análises utilizando o procedimento A. inicialmente verificamos que em todos os esquemas de perturbação o teste geral foi significante segundo o teste de máxima inclinação e/ou máxima curvatura. Desta forma, utilizamos os valores críticos relativos ao passo 2 do procedimento $\mathrm{A}$.

Apresentamos os valores das estatísticas propostas em todos os casos de perturbação, mas vale ressaltar que as observações consideradas influentes são aquelas em que apresentam valor da estatística significante em pelo menos um esquema de perturbação apresentado. 


\begin{tabular}{|c|c|c|c|c|c|c|c|}
\hline \multirow[b]{3}{*}{ Data } & \multirow[b]{3}{*}{ Localização } & \multicolumn{4}{|c|}{ Perturbação do Modelo } & \multirow{2}{*}{\multicolumn{2}{|c|}{ Perturbação nos Dados }} \\
\hline & & \multicolumn{2}{|c|}{ Volatilidade } & \multicolumn{2}{|c|}{ Aditiva } & & \\
\hline & & Inclinação & Curvatura & Inclinação & Curvatura & Inclinação & Curvatura \\
\hline $20 / 10 / 1997$ & 201 & 0.1071 & 0.2586 & 0.0166 & 0.1619 & 0.0639 & 0.1268 \\
\hline $21 / 10 / 1997$ & 202 & 0.1044 & 0.2750 & 0.0258 & 0.1716 & 0.0620 & 0.1243 \\
\hline $23 / 10 / 1997$ & 204 & 0.1030 & 0.2477 & 0.0253 & 0.1225 & 0.0610 & 0.1321 \\
\hline $24 / 10 / 1997$ & 205 & 0.0663 & 0.1267 & 0.0136 & 0.0809 & 0.1112 & 0.2427 \\
\hline $08 / 05 / 1998$ & 337 & 0.1060 & 0.2281 & 0.0188 & 0.0159 & 0.0631 & 0.1363 \\
\hline $03 / 06 / 1998$ & 355 & 0.1808 & 0.2317 & 0.0318 & 0.0118 & 0.0932 & 0.1290 \\
\hline $26 / 08 / 1998$ & 414 & 0.1295 & 0.2735 & 0.0524 & 0.0946 & 0.0754 & 0.1255 \\
\hline $27 / 08 / 1998$ & 415 & 0.1106 & 0.2833 & 0.0395 & 0.0906 & 0.0660 & 0.1271 \\
\hline $28 / 08 / 1998$ & 416 & 0.0994 & 0.2863 & 0.0559 & 0.0921 & 0.0582 & 0.1262 \\
\hline $31 / 08 / 1998$ & 418 & 0.1086 & 0.2863 & 0.0363 & 0.0775 & 0.0648 & 0.1268 \\
\hline $03 / 09 / 1998$ & 420 & 0.1258 & 0.2801 & 0.0603 & 0.0395 & 0.0738 & 0.1285 \\
\hline $17 / 12 / 1998$ & 492 & 0.1022 & 0.2381 & 0.0523 & 0.0269 & 0.0604 & 0.1364 \\
\hline $23 / 07 / 1999$ & 640 & 0.0996 & 0.2279 & 0.1139 & 0.0203 & 0.0584 & 0.1270 \\
\hline $26 / 07 / 1999$ & 641 & 0.2476 & 0.2195 & 0.0322 & 0.0108 & 0.1099 & 0.1306 \\
\hline $27 / 09 / 1999$ & 685 & 0.1882 & 0.2227 & 0.0159 & 0.0262 & 0.0953 & 0.1260 \\
\hline $29 / 03 / 2000$ & 812 & 0.1034 & 0.2598 & 0.0285 & 0.0644 & 0.0613 & 0.1269 \\
\hline $30 / 03 / 2000$ & 813 & 0.1966 & 0.2651 & 0.0113 & 0.0466 & 0.0976 & 0.1279 \\
\hline $18 / 04 / 2000$ & 826 & 0.1065 & 0.2152 & 0.0105 & 0.0119 & 0.0634 & 0.1434 \\
\hline $19 / 04 / 2000$ & 827 & 0.1040 & 0.2125 & 0.0099 & 0.0257 & 0.0618 & 0.1435 \\
\hline $20 / 06 / 2000$ & 869 & 0.1134 & 0.2823 & 0.0311 & 0.0764 & 0.0676 & 0.1282 \\
\hline $23 / 06 / 2000$ & 871 & 0.1034 & 0.2859 & 0.0244 & 0.0714 & 0.0613 & 0.1298 \\
\hline $04 / 07 / 2000$ & 878 & 0.1336 & 0.2231 & 0.0807 & 0.0133 & 0.0772 & 0.1310 \\
\hline $06 / 07 / 2000$ & 880 & 0.1160 & 0.2097 & 0.1127 & 0.0244 & 0.0690 & 0.1279 \\
\hline $03 / 08 / 2000$ & 900 & 0.1032 & 0.2819 & 0.0245 & 0.0723 & 0.0612 & 0.1289 \\
\hline $13 / 09 / 2000$ & 928 & 0.1230 & 0.2029 & 0.1595 & 0.0033 & 0.0725 & 0.1285 \\
\hline $14 / 09 / 2000$ & 929 & 0.1822 & 0.2057 & 0.0851 & 0.0020 & 0.0936 & 0.1285 \\
\hline $08 / 08 / 2001$ & 1153 & 0.1099 & 0.2142 & 0.1242 & 0.0036 & 0.0656 & 0.1334 \\
\hline $09 / 08 / 2001$ & 1154 & 0.1990 & 0.2175 & 0.0640 & 0.0036 & 0.0982 & 0.1292 \\
\hline $12 / 09 / 2001$ & 1180 & 0.1030 & 0.2184 & 0.0403 & 0.1075 & 0.0610 & 0.1281 \\
\hline $14 / 09 / 2001$ & 1182 & 0.0562 & 0.1267 & 0.0410 & 0.0331 & 0.0991 & 0.2205 \\
\hline Máximo & & 5315.13 & 97.71 & 5832.50 & 226.15 & 5629.48 & 123.95 \\
\hline$T_{0}$ & & 2648.07 & 115.63 & 5448.58 & 165.85 & 5568.33 & 134.73 \\
\hline$T_{1}$ & & 0.1815 & 0.2300 & 0.1020 & 0.1195 & 0.0935 & 0.1360 \\
\hline$T_{2}$ & & 0.3867 & 0.4124 & 0.1298 & 0.2270 & 0.2667 & 0.2424 \\
\hline
\end{tabular}

Tabela 5.6: Diagnóstico baseado na curvatura e inclinação para NYSE.

Na Tabela 5.6, máximo refere-se a máxima inclinação e curvatura sob cada esquema de perturbação. $T_{0}$ é o limiar da máxima inclinação ou máxima curvatura ao n.s. de $5 \%$. $T_{1}$ é o limiar obtido através de interpolação entre os limiares do tamanho de amostra 1000 e 1500 na Tabela 5.2 , e $T_{2}$ é o limiar obtido pela tabela 5.4. Os maiores componentes do vetor diagnóstico estão em negrito, e suas observações correpondentes são identificadas como influentes pelo gráfico de influência

Pode-se verificar através da Tabela 5.6 que o método caracterizou como influentes todas as observações associadas às crises do mercado. Veja que, se analisarmos os 
diferentes tipos de perturbação separadamente os resultados mostram que o método detecta maior número de observações sob o esquema de perturbação na volatilidade. Por outro lado, a estatística de curvatura sob a perturbação aditiva é a que detecta menor número de observações.

\subsection{Conclusões}

Neste capítulo analisamos a influência local no modelo de volatilidades estocástica baseado na verossimilhança deslocada usando a inclinação e curvatura. Encontramos valores de limiares para a caracterização de valores influentes através de simulação de Monte Carlo e aplicamos o método a duas séries de dados reais. Os resultados obtidos foram consistentes com os encontrados na literatura e uma breve comparação com a análise do modelo GARCH feita por Zhang e King (2005) será apresentada no capítulo 6 . 



\section{CAPÍTULO 6}

\section{Conclusão}

Neste trabalho foram apresentados métodos para análise de valores atípicos em séries financeiras. Para as análises, foi utilizado o modelo de volatilidade estocástica sob a abordagem bayesiana e freqüentista.

Foram apresentados três métodos para auxiliar na caracterização da influência ou não de uma determinada observação. Inicialmente, o objetivo da tese não é tratar as metodologias de forma comparativa e sim obter critérios complementares para a caracterização de uma observação na série observada.

No Capítulo 3 verificamos a eficiência do tratamento bayesiano quando consideramos a deteç̧ão de outliers. Constatamos que a metodologia é eficaz para a deteç̧ão de valores atípicos quando esses ocorrem isolados ou em blocos. As simulações mostraram que os valores considerados outliers são detectados com alta probabilidade a posteriori. As análises das séries reais mostraram-se consistentes com os resultados obtidos através de outras metodologias existentes na literatura. 
As análises dos valores atípicos sob o enfoque freqüentista foram baseadas em estudos de análise de influência e influência local. Portanto, essas metodologias caracterizam as observações considerando a influência de uma forma geral, ou seja, são considerados influentes não apenas valores que são considerados outliers.

No Capítulo 4 apresentamos uma estatística para a determinação da influência das observações. Verificamos que o método proposto foi eficaz na caracterização de outliers quando ocorrem isolados e em blocos. Foi visto que a estatística também ressalta os valores influentes além daqueles que são caracterizados como outliers.

Portanto, analisando os Capítulos 3 e 4 verificamos que os valores atípicos encontrados pelo procedimento bayesiano geralmente são detectados pela estatística de influência. Lembrando que o procedimento bayesiano tem como objetivo detectar os valores que iremos considerar como um outlier ou um bloco de outlier e no Capítulo 4 caracterizamos não só os outliers.

Finalmente, verificamos que a análise através da metodologia de infuência local é a mais sensível das três na caracterização de uma observação. Mas, vale ressaltar que essa característica pode não ser desejada em alguns casos e que sua eficiência é levemente inferior ao método bayesiano quando tratamos outliers.

Lembremos que no método bayesiano temos a desvantagem do custo computacional. Por outro lado, a implementação simples da metodologia baseada na estatística de influência é um grande atrativo para o método. Mas, devemos considerar que a estatística de influência obtida a partir de estimativas baseadas na verossimilhança exata poderia tornar o método um pouco mais oneroso quando tratamos da implementação e do tempo computacional. 
Os benefícios obtidos ao considerar estimativas através da verossimilhança exata, seriam estudos a serem considerados futuramente. Um outro fato a destacar, é a comparação entre o tempo computacional do método de influência e influência local, o que também não foi verificado porque no método de influência local as estimativas foram obtidas pelo método de verossimilhança exata e a estatística de influência foi baseada na quasi-verossimilhança.

As Tabelas 6.1 e 6.2 apresentam os valores caracterizados como atípicos sob as três metodologias apresentadas. As tabelas possuem uma marcação (Y) para os valores que foram considerados atípicos sob a metodologia em análise.

Na Tabela 6.1 podemos ressaltar os seguintes períodos:

- Entre 08 e 10 de Março de 1995 foi período de introdução de intrabandas cambiais.

- Em 15 de Julho de 1997 tivemos período da crise da Ásia I.

- No período de 21 de outubro de 1997 e por volta de 12 de novembro ocorreu a crise da Ásia II.

- Por volta de 25 de agosto de 1998 foi o período em que a Rússia decretou a moratória.

- Em torno de 10 de setembro de 1998 tivemos uma crise econômica mundial, quando as bolsas de valores em todo o mundo despencaram e houve aumento generalizado das taxas de juros domésticas.

- Em 15 de janeiro de 1999 houve a desvalorização do real.

- Em torno de 17 de abril de 2000 houve a crise da Nasdaq. 


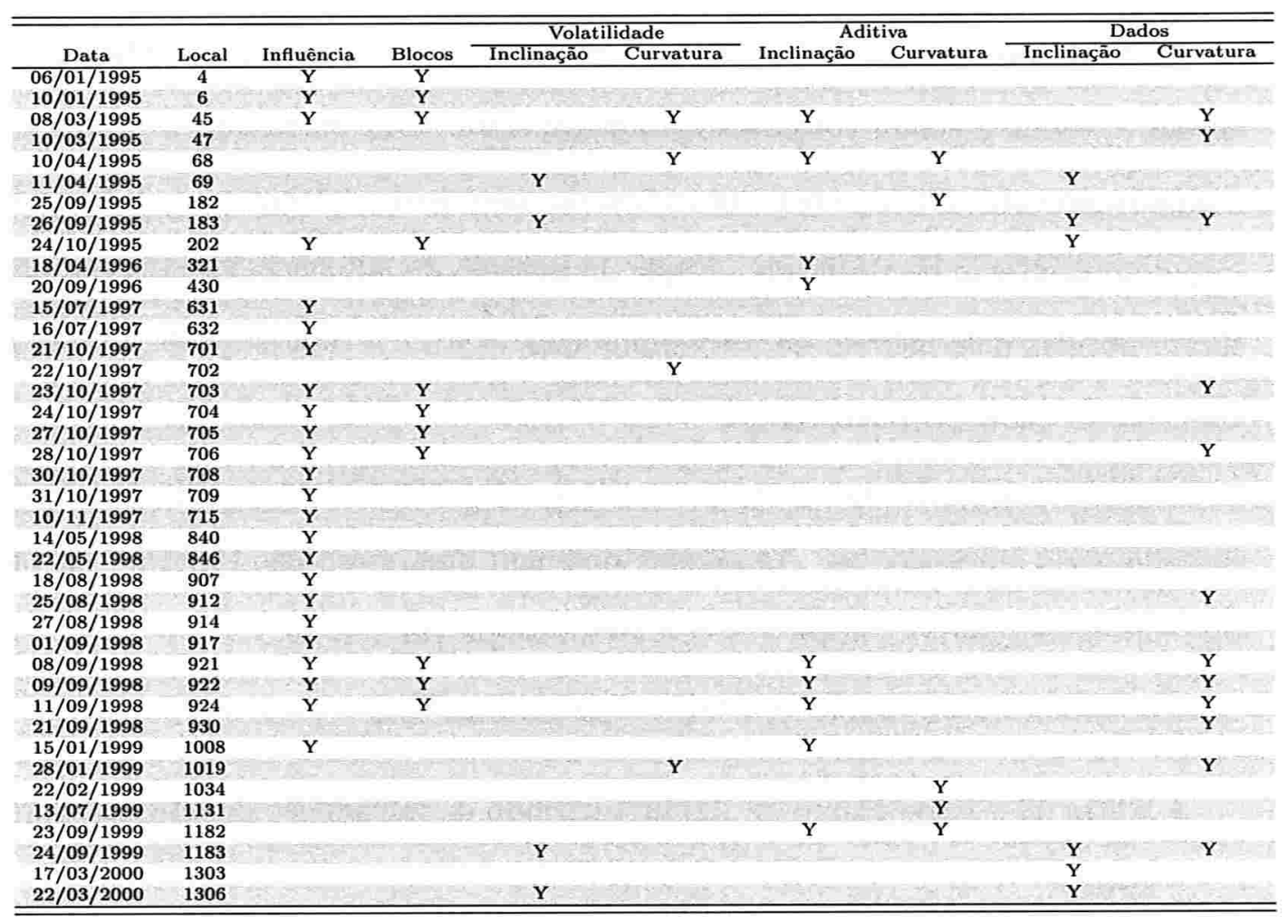

Tabela 6.1: Comparação de métodos para série do Ibovespa.

Considerando a série do Ibovespa, podemos verificar que praticamente todos os valores caracterizados estão relacionadas às crises apresentadas no Capítulo 2 . 
Analisando a Tabela 6.2 também podemos ressaltar alguns fatos relevantes no período de observação da série. Neste caso podemos citar :

- Período de 20 a 28 de outubro de 1997 foi período em que ocorreu a crise da Ásia II.

- Entre 26 de agosto e 03 de setembro de 1998 foi decretada a moratória da Rússia.

- Em torno de 17 a 22 de abril de 2000 houve a crise da Nasdaq.

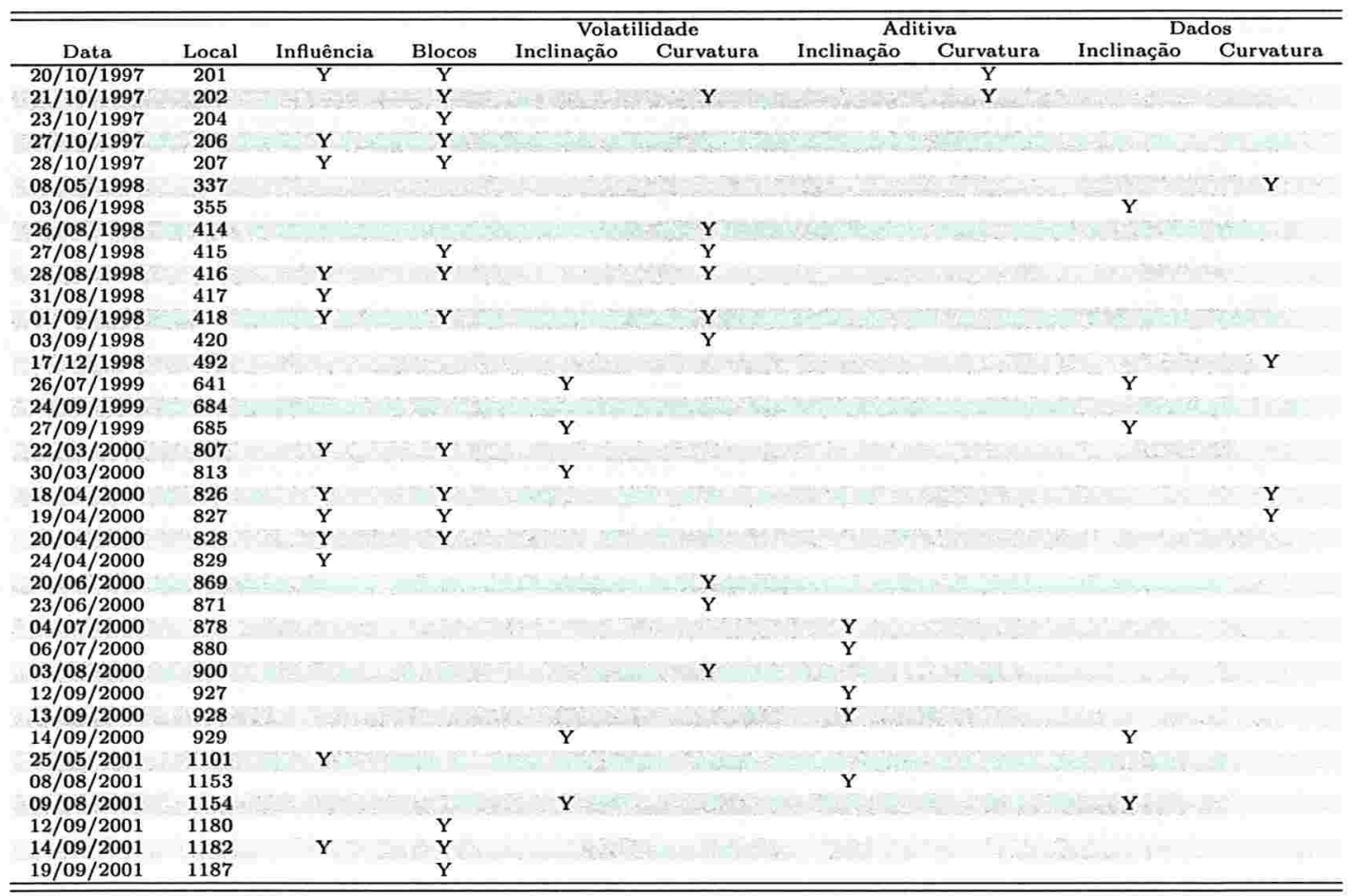

Tabela 6.2: Comparação de métodos para série do NYSE. 
As Tabelas 6.3 e 6.4 apresentam uma breve comparação entre os métodos. Note que, na tabela consideramos uma coluna referente à análise de influência local e esta coluna representa a caracterização de observação influente sob pelo menos um esquema de perturbação.

Na diagonal encontramos o número de observações detectada apenas pelo método em questão, por exemplo, na linha 1 e coluna 1 da Tabela 6.3 temos que na série de retornos da NYSE o método de influência proposto no Capítulo 4 detectou como influentes um total de 13 observações. Na primeira linha e segunda coluna encontramos o número de observações que foram detectadas tanto pela estatística de influência como pelo método bayesiano apresentado no Capítulo 3.

\begin{tabular}{cccccccc}
\hline \hline & Influência & Blocos & Local & Volatilidade & Aditiva & Dados & Zhang \\
\hline Influência & 13 & 10 & 5 & 2 & 1 & 2 & 8 \\
Blocos & - & 15 & 7 & 4 & 2 & 2 & 11 \\
Local & - & - & 26 & 14 & 8 & 9 & 6 \\
Volatilidade & - & - & - & 14 & 1 & 4 & 4 \\
Aditiva & - & - & - & - & 8 & 0 & 2 \\
Dados & - & - & - & - & - & 9 & 1 \\
Zhang & - & - & - & - & - & - & 12 \\
\hline \hline
\end{tabular}

Tabela 6.3: Comparação da quantidade de valores detectados para série do NYSE.

\begin{tabular}{ccccccc}
\hline \hline & Influência & Blocos & Local & Volatilidade & Aditiva & Dados \\
\hline Influência & 25 & 11 & 9 & 1 & 5 & 8 \\
Blocos & - & 11 & 7 & 1 & 4 & 7 \\
Local & - & - & 25 & 8 & 12 & 16 \\
Volatilidade & - & - & - & 8 & 2 & 6 \\
Aditiva & - & - & - & - & 12 & 4 \\
Dados & - & - & - & - & - & 16 \\
\hline \hline
\end{tabular}

Tabela 6.4: Comparação da quantidade de valores detectados para série do Ibovespa. 
Resumidamente, os métodos produziram bons resultados nas análises de séries financeiras sob o modelo de volatilidade estocástica e no caso da escolha de um único procedimento devemos considerar o objetivo da análise.

A análise de outliers em séries financeiras tem sido objeto de estudos de diversos pesquisadores. Visto que, o mercado financeiro tem utilizado constantemente os modelos de volatilidade estocástica, naturalmente pesquisas dos modelos em questão estão avançando.

Estudos relacionados ao poder dos testes apresentados assim como a comparação entre os métodos propostos são importantes para o desenvolvimento da metodologia. As propriedades da estatística de influência apresentada no Capítulo 4 também seria objeto de estudo futuro.

A maior parte das pesquisas em séries financeiras trabalham com outliers do tipo aditivo. Estudos considerando a extensão das metodologias apresentadas neste trabalho à outliers de inovação seria importante a serem consideradas em trabalhos futuros. 



\section{REFERÊNCIAS}

[1] Atkinson, A. C. (1986). Masking and unmasked. Biometrika, 73:533-541.

[2] Barnett, G., Kohn, R. e Sheather, S. (1996). Bayesian estimation of an autoregressive model using markov chain monte carlo. Journal of Econometrics, 74:237-254.

[3] Barnett, G., Kohn, R. e Sheather, S. (1997). Robust bayesian estimation of an autoregressive-moving average models. Journal of Time Series Analysis, 18:11-28.

[4] Belsley, D. A., Kuh, E. e Welsch, R. E. (1980). Regression Diagnostics: Identifying Influential Data and Sources of Collinearity. New York: John Wiley.

[5] Billor, N. e Loynes, R. M. (1993). Local influence: A new approach. Communications in Statistics, Theory and Methods, 22:1595-1611.

[6] Bollerslev, T. (1986). Generalized autoregressive conditional heteroskedasticity. Journal of Econometrics, 31:307-327.

[7] Bollerslev, T., Chou, R. Y. e Kroner, K. F. (1992). ARCH modelling in finance: A review of theory and empirical evidence. Journal of Econometrics, 52:5-59. 
[8] Bollerslev, T., Chou, R. Y. e Kroner, K. F. (1994). ARCH modelling in finance: A review of theory and empirical evidence. Journal of Econometrics, 52:5-59.

[9] Box, G. E. P. e Tiao, G. C. (1968). A bayesian approach to some outlier problems. Biometrika, 55:119-129.

[10] Brown, G. C. e Lawrence, A. J. (2000). Theory and illustration of regression influence diagnostics. Communications in Statistics, Part A - Theory and Methods, 29:2079-2107.

[11] Chang, C., Tiao, G. e Chen, C. (1988). Estimation of time series parameters in the presence of outliers. Technometrics, 3:193-204.

[12] Chen, C. e Liu, L. (1993). Joint estimation of model parameters and outliers effects in time series. Journal of the American Statistical Association, 88:284-297.

[13] Chib, S., Nardari, F. e Shephard, N. (2002). Markov chain monte carlo methods for stochastic volatility models. Journal of Econometrics, 108:281-316.

[14] Cook, R. D. (1977). Detection of influential observations in linear regression. Technometrics, 19:15-18.

[15] Cook, R. D. (1986). Assessment of local influence. Journal of Royal Statistical Society (Série B), 48:133-169.

[16] Cook, R. D., Peña, D. e Weisberg, S. (1988). The likelihood displacement: A unifying principle for influence. Communications in Statistics, Part A - Theory and Methods, 17:623-640. 
[17] Cook, R. D. e Weisberg, S. (1982). Residuals and Influence in Regression. London: Chapman and Hall.

[18] de Jong, P. (1988). The likelihood for state space models. Biometrika, 75:165169.

[19] de Jong, P. e Shephard, N. (1995). The simulation smoother for time series models. Biometrika, 82:339-350.

[20] Doornik, J. A. (1999). OX an Object-Oriented Matrix Programming Language. London: Timberlake Consults Press.

[21] Durbin, J. e Koopman, S. J. (1997). Monte Carlo maximum likelihood estimation for non-gaussian state space models. Biometrika, 84:669-684.

[22] Durbin, J. e Koopman, S. J. (2000). Time series analysis of non-gaussian observations based on state space models from both classical and bayesian perspectives. Journal of the Royal Statistical Society (Série B), 62:3-56.

[23] Engle, R. F. (1982). Autoregressive conditional heteroskedasticity with estimates of the variance of UK inflation. Econometrica, 50:987-1008.

[24] Fox, A. J. (1972). Outliers in time series. Journal of the Royal Statistical Society (Série B), 34:350-363.

[25] Fukui, P. (2000). Deteç̧ão de valores aberrantes em modelos de volatilidade estocástica. Tese de mestrado, Departamento de Estatística, UNICAMP. 
[26] Fung, W. K. e Tang, M. K. (1997). Assessment of local influence in multivariate regression analysis. Communications in Statistics - Theory and Methods, 26,4:821837.

[27] Galea, M., Paula, G. A. e Bolfarine, H. (1997). Local influence in elliptical regression models. The Statistician, 46:71-79.

[28] Gilks, W., Best, N. e Tan, K. (1995). Adaptative rejection Metropolis sampling within Gibbs sampling. Applied Statistics, 44(4):455-472.

[29] Harvey, A. C. (1989). Forecasting, Structural Time Series Models and the Kalman Filter. Cambridge: Cambridge University Press.

[30] Harvey, A. C. e Koopman, S. J. (1992). Diagnostic checking of unobservedcomponents time series models. Journal of Business and Economic Statistics, 10:377-389.

[31] Harvey, A. C., Ruiz, E. e Shephard, N. (1994). Multivariate stochastic variance models. Review of Economics Studies, 61:247-264.

[32] Hotta, L. K. e Tsay, R. S. (1998). Outliers in GARCH processes. Graduate School of Business, University of Chicago.

[33] Hull, J. e White, A. (1987). The pricing of options on assets with stochatic volatilities. Journal of Finance, 42:281-300.

[34] Jacquier, E., Polson, N. e Rossi, P. E. (1994). Bayesian analysis of stochastic volatility models (with discussion). Journal of Business and Economic Statistics, $12: 371-417$. 
[35] Jacquier, E., Polson, N. e Rossi, P. E. (1995). Priors and models for multivariate stochastic volatility. Graduate School of Business, University of Chicago.

[36] Jacquier, E., Polson, N. e Rossi, P. E. (2004). Bayesian analysis of stochastic volatility models with fat-tail and correlated errors. Journal of Econometrics, 122:185-212.

[37] Jiancheng, J. e Xizhi, W. (1998). Assessment of local influence on cox's proportional hazard model. Acta Mathematicae Applicatae Sinica, 14, (4):414-424.

[38] Jungbacker, B. e Koopman, S. J. (2006). On importance sampling for state space models. Vrije Universiteit Amsterdam, Department of Econometrics and Operations Research.

[39] Justel, A., Peña, D. e Tsay, R. S. (2001). Detection of outliers patches in autoregressive time series. Statistica Sinica, 11:651-673.

[40] Kalman, R. E. (1960). A new approach to linear filtering and prediction problems. Journal of Basic Engineering, Transactions ASMA, Series D, 82:35-45.

[41] Kim, C. (1996). Local influence and replacement measure. Communications in Statistics - Theory and Methods, 25,1:49-61.

[42] Kim, M. G. (1995). Local influence in multivariate regression. Communications in Statistics - Theory and Methods, 20:1271-1278.

[43] Kim, S., Shephard, N. e Chib, S. (1998). Stochastic volatility: Likelihood inference and comparison with ARCH models. Review of Economic Studies, 85:361393. 
[44] Koopman, S. J. (1993). Disturbance smoother for state space models. Biometrika, 80:117-126.

[45] Koopman, S. J., Shephard, N. e Doornik, J. A. (1999). Statistical algorithms for models in state space form using ssfpack 2.2. Econometrics Journal, 2:113-166.

[46] Laurent, R. e Cook, R. (1993). Leverage, local influence and curvature in nonlinear regression. Biometrika, 80:99-106.

[47] Lee, A. H. e Zhao, Y. (1996). Assessing local influence in measurement error models. Biometrical Journal, 38:829-841.

[48] Lesafre, E. e Verbeke, G. (1998). Local influence in linear mixed models. Biometrics, 54:570-582.

[49] Liesenfeld, R. e Richard, J. F. (2003). Univariate and multivariate stochastic volatility models: Estimation and diagnostic. Journal of Empirical Finance, 207:127.

[50] Liu, S. (2004). On diagnostics in conditionally heteroskedastic time series models under elliptical distributions. Applied Probability Trust, 1:393-415.

[51] McCulloch, R. E. e Tsay, R. S. (1994). Bayesian analysis of autoregressive time series via the Gibbs sampler. Journal of Time Series Analysis, 15:235-250.

[52] Motta, A. C. O. e Hotta, L. K. (2003). Exact maximum likelihood and bayesian estimation of the stochastic volatility model. Brazilian Review of Econometrics, 23:183-226. 
[53] Omori, Y., Chib, S., Shephard, N. e Nakajima, J. (2005). Stochastic volatility with leverage: Fast and efficient likelihood inference. Working Paper, Universidade de Tokio, Faculdade de Economia.

[54] Pan, J., Fang, K. e Liski, E. (1996). Bayesian local influence for the growth curve model with Rao's simple covariance structure. Journal of Multivariate Analysis, 58:55-81.

[55] Paula, G. (1996). Influence diagnostics in proper dispersion models. Australian Journal of Statistics, 38:307-316.

[56] Peña, D. (1990). Influential observations in time series. Journal of Business and Economic Statistics, 8:235-241.

[57] Peña, D. (2005). A new statistic for influence in linear regression. Technometrics, $47: 1-12$.

[58] Penzer, J. (2006). State space models for time series with patches of unusual observations. Forthcoming.

[59] Pregibon, D. (1981). Logistic regression diagnostic. The Annals of Statistics, 9:705-724.

[60] Ruiz, E. (1994). Quasi-maximum likelihood estimation of stochastic variance models. Journal of Econometrics, 63:284-306.

[61] Shephard, N. e Pitt, M. K. (1997). Likelihood analysis of non-gaussian measurement time series. Biometrika, 84:653-667. 
[62] Sánchez, M. . J. e Peña, D. (2003). The identification of multiple outliers in arima models. Communications in Statistics: Theory and Methods, 32:1265-1287.

[63] Taylor, S. J. (1986). Modelling Financial Time Series. Chichester: John Wiley.

[64] Taylor, S. J. (1994). Modelling stochastic volatility: A review and comparative study. Mathematical Finance, 4:183-204.

[65] Thomas, W. e Cook, R. D. (1990). Assessing influence on predictions from generalized linear models. Technometrics, 32:59-65.

[66] Tsay, R. S. (1986). Time series model specification in the presence of outliers. Journal of the American Statistical Association, 81:132-141.

[67] Tsay, R. S. (1988). Outliers, level shifts, and variance change in time series. Journal of Forecasting, 7:1-20.

[68] Tsay, R. S., Peña, D. e Pankratz, A. (1998). Outliers in multivariate time series. Working Paper, Universidade Carlos III Madrid, pages 98-96.

[69] van Dijk, D., Franses, P. H. e Lucas, A. (1999). Testing for ARCH in the presence of additive outliers. Journal of Applied Econometrics, 14:539-562.

[70] Williams, D. A. (1987). Generalized linear model diagnostics using the deviance and single case deletions. Applied Statistics, 36:181-191.

[71] Wu, X. e Luo, Z. (1993). Second-order approach to local influence. Journal of Royal Statistical Society (Série B), 55:929-936.

[72] Zhang, X. (2004). Assessment of local influence in garch processes. Journal of Time Series Analysis, 25(2):301-313. 
[73] Zhang, X. e King, M. L. (2005). Influence diagnostic in GARCH processes. Journal of Business and Economic Statistics, 23:118-129. 